\title{
A geometric classification of Lie groups
}

Nick Th. Varopoulos

\section{OV. An overview.}

\section{OV.1. The scope of this overview.}

This paper is part of a general program that was originally designed to study the "Heat diffusion kernel on Lie groups". The scope of this introductory section is the following:

i) Explain in general terms and with emphasis on intuition, what this program is about, and explain how this program fits in the general context of Lie groups.

ii) Explain how the present paper fits in this program.

iii) This introductory section is addressed to non experts. The only prerequisite that is needed is the definition of a Lie group and its Haar measure, and the definition of the convolution of measure on such a group. The definition of the Lie algebra and of a soluble Lie algebra will be given in Section OV.3 below. I will not give the definition of the Heat diffusion semigroup $T_{t}=e^{-t \Delta}$ that appears in Section OV.2, but the reader could either ignore this and concentrate on convolutions of measures, or could refer to [17] for a formal definition. If any other unknown words crop up the reader should disregard them and move on.

iv) The price that inevitably had to be paid for making this overview accessible to the "general public" is in the precision and even the 
accuracy of the presentation. In fact some of the assertions made in this overview are, as such incorrect. But these inaccuracies can easily be corrected, and this is done in the course of the paper.

v) At the end of Part 0 of this paper I shall give a "guide to the reader" that is quite detailed, and where a serious effort is made to help the reader who wishes to "grind" his way through the proofs.

\section{OV.2. The previous work in the area.}

Let $G$ be some locally compact group and let $d \mu(x)=\varphi(x) d x$ be some probability measure, where $\varphi \in C_{0}(G)$ is continuous with compact support, where $d x$ is the left Haar measure and where $d \mu\left(x^{-1}\right)=d \mu(x)$ (i.e. the mapping $x \longrightarrow x^{-1}$ stabilizes $\mu$ ). We shall consider the convolution powers of $\mu$

$$
d \mu^{* n}(x)=\varphi_{n}(x) d x, \quad n \geq 1 .
$$

We shall fix $g \in G$, say $g=e \in G$, the neutral element, and consider

$$
\phi(n)=\varphi_{n}(g), \quad n \geq 1 .
$$

It is a central issue to study the behaviour of $\phi(n)$ as $n \longrightarrow \infty$. Indeed, apart from its intrinsic interest, the behaviour of $\phi(n)$ controls the analysis and the geometry of $G$. The reader could think of the Heat or the Poisson convolution semigroups on $G=\mathbb{R}^{d}$

$$
\begin{gathered}
H_{t}(x)=c t^{-d / 2} \exp \left(-\frac{|x|^{2}}{4 t}\right), \\
P_{t}(x)=\frac{c t}{\left(t^{2}+|x|^{2}\right)^{(d+1) / 2}},
\end{gathered}
$$

and refer to the classical literature in Real Analysis (cf. [19]) where these semigroups are used systematically to prove geometric results, such as the Sobolev inequalities and such like. The same analysis can be made on a general Lie group $G$ by considering the generalized Heat diffusion semigroup $T_{t}=e^{-t \Delta}$, where $\Delta=-\Sigma X_{j}^{2}$ is a generalized Laplacian ( $c f .[17]) . T_{t}$ is then, as in the classical case, a convolution semigroup: $T_{t_{1}} * T_{t_{2}}=T_{t_{1}+t_{2}}$. From this the importance of $\phi(n)$ in (OV.2) becomes amply apparent. 
Much progress on the above problem was made in the decade 198090 , and this was reported in the book [17]. The main geometric invarient used in that approach was the volume growth of the group

$$
\gamma(n)=\text { Haar measure }\left(\Omega^{n}\right), \quad n \geq 1,
$$

where $e \in \Omega=\Omega^{-1}$ is some compact neighbourhood of the neutral element $e \in G$. What emerges is that, for unimodular locally compact groups (i.e. for the groups where the left and the right Haar measures coinside, e.g. discrete groups), we have the following dichotomy:

$\left.\mathrm{D}_{1}\right)$ If $\gamma(n) \geq e^{c n}$ for some $c>0$, i.e. if $\gamma(n)$ grows as fast as an exponential, then

$$
\phi(n)=O\left(e^{-c_{1} n^{1 / 3}}\right),
$$

for some $c_{1}>0$. The above is sharp, and perhaps also, at first sight, surprising.

$\left.\mathrm{D}_{2}\right)$ If $\gamma(n) \approx n^{D}$, then

$$
\phi(n) \approx n^{-D / 2}
$$

as one would expect from the classical case $G=\mathbb{R}^{d}$.

The unimodularity is essential for the above dichotomy. Indeed every non unimodular group can immediately be seen to satisfy $\gamma(n) \geq$ $e^{c n}$, and yet the simplest non abelian Lie group of affine transformations on $\mathbb{R}$

$$
x \longmapsto a x+b, \quad a>0, b \in \mathbb{R},
$$

satisfies $\phi(n) \approx n^{-3 / 2}(c f .[20])$. That group is of course not unimodular.

The scope of the above program can be described by saying that we want to find the analog of the above classification for all Lie groups and not just the unimodular ones.

\section{OV.3. The Lie algebra.}

The dichotomy described in Section OV.2 holds for all locally compact groups and not only Lie groups. If $G$ is a connected Lie group we can go much further because we have at our disposal the very powerful tool of the Lie algebra $\mathfrak{g}$ of $G$. This is the finite dimension vector space 
(in 1-1 correspondence with $T_{e}(G)$ the tangent space at $e$ ) of all vector fields on $G$ that are invariant by the left action of $G$. $\mathfrak{g}$ then admits the natural algebra structure that is induced by the bracket operation on vector fields

$$
[X, Y] f=(X Y-Y X) f, \quad f \in C^{\infty}(G), X, Y \in \mathfrak{g}
$$

It is customary and convenient to define then

$$
\begin{gathered}
\operatorname{ad}(x): \mathfrak{g} \longrightarrow \mathfrak{g}, \quad \operatorname{ad}(x) \in \mathcal{L}(\mathfrak{g}), \\
\operatorname{ad}(x) y=[x, y] \text { the algebra multiplication. }
\end{gathered}
$$

One says that $\mathfrak{g}$ is an $R$-algebra if all the eigenvalues of ad $(x)(x \in \mathfrak{g})$ are pure imaginary. One also says that $\mathfrak{g}$ and $G$ are soluble if it is possible to find a basis in the complexified $\mathfrak{g}_{c}=\mathfrak{g} \otimes_{\mathbb{R}} \mathbb{C}$, with respect to which all the ad-mappings (OV.4) become simultaneously upper triangular ( $c f$. $[1],[9])$

$$
\operatorname{ad}(x)=\left(\begin{array}{ccc}
\lambda_{1}(x) & & * \\
& \ddots & \\
0 & & \lambda_{k}(x)
\end{array}\right), \quad k=\operatorname{dim} \mathfrak{g}, \quad \lambda_{j} \in \mathfrak{g}_{c}^{*} .
$$

For soluble algebras the following classification is crucial: ( $c f$. Section $0.1,[18],[21])$. Let $\mathcal{L}=\left(L_{1}, \ldots, L_{s}\right), \lambda \subset \mathfrak{g}^{*}$ the distinct non zero $\operatorname{Re} \lambda_{j}$ $(1 \leq j \leq k)$ (if $G$ is an $R$-group then the above set is empty).

C) We say that $G$ is $\mathrm{C}$ if $\mathcal{L} \neq \varnothing$ and if there exists $\alpha_{j} \geq 0$ such that

$$
\sum_{j=1}^{s} \alpha_{j}=1, \quad \sum_{j=1}^{s} \alpha_{j} L_{j}=0, \quad 1 \leq j \leq s .
$$

NC) We say that $G$ is $\mathrm{NC}$ if it is not C.

\section{OV.4. The Algebraic-Geometric Dichotomy ([22]).}

For a connected Lie group if we use the Lie algebra we can complete the classification of Section OV.2 by the following Theorem of Y. Guivarc'h ([22]).

$\left.\mathrm{D}_{1}\right) \gamma(n) \geq e^{c n}$ if and only if $\mathfrak{g}$ is not an $R$-algebra. 
$\left.\mathrm{D}_{2}\right) \gamma(n) \approx n^{D}$ if and only if $\mathfrak{g}$ is an $R$-algebra.

If we restrict ourselves to unimodular connected Lie groups, it follows ( $c f .[17])$ that they can be classified into two classes and that the classification is:

i) Geometric: By means of the growth of $\gamma(n)$.

ii) Algebraic: By means of the $R$-condition on the Lie algebra. OV.2).

iii) Analytic: By means of the behaviour at infinity of $\phi(n)$ ( $c f$.

\section{OV.5. The General Analytic classification.}

The first step towards the extension of the classification of Section OV.4 to a general connected real Lie group was taken in [2], [21]. We classified these any such Lie group $G$ into two classes, the B-groups and the NB-groups, and we proved:

B) If $G$ is a B-group and $\mu \in \mathbb{P}(G)$ is as in Section OV.1, then there exists $\lambda=\lambda(\mu) \geq 0$ (that depends on $\mu$ ) and $C_{i}, c_{i}>0, i=1,2$, such that the corresponding $\phi(n)$ ( $c f$. Section OV.2) satisfies

$$
C_{2} e^{-\lambda n-c_{2} n^{1 / 3}} \leq \phi(n) \leq C_{1} e^{-\lambda n-c_{1} n^{1 / 3}}, \quad n \geq 1
$$

NB) If $G$ is a NB-group and $\mu \in \mathbb{P}(G)$ is as in Section OV.1, then there exists $\lambda=\lambda(\mu) \geq 0$ and $\nu \geq 0$ (that both depend on $\mu$ ) and $C_{i}>0, i=1,2$, such that

$$
C_{2} e^{-\lambda n} n^{-\nu} \leq \phi(n) \leq C_{1} e^{-\lambda n} n^{-\nu}, \quad n \geq 1 .
$$

In both the B and NB case either for all $\mu \in \mathbb{P}(G)$ we have $\lambda(\mu)=0$, and then we say that $G$ is amenable, or $\lambda(\mu)>0$, and then we say that $G$ is non amenable ( $c f .[23])$.

\section{OV.6. The Algebraic classification.}

Let $G$ be some connected Lie group, then we can find $R \subset G$ some closed connected soluble subgroup and $K$ some compact subgroup such that $G=R K$ : This statement is almost correct but not quite. It is 
essentially an abused form of the Borel decomposition (cf. [24]) - Ignore this difficulty but observe that unless $G$ is amenable $R$ cannot be chosen to be a normal subgroup. We have:

B) If $G$ is a B-group then every soluble subgroup $R$ as above is a C-group.

NB) If $G$ is a NB-group then every subgroup $R$ as above is NC. This is the main result in [2], [18], cf. [21].

Furthermore it is easy to see that the above classification is purelly algebraic, i.e. it only depends on $\mathfrak{g}$, the Lie algebra of $G$ ( $c f$. [2]).

\section{OV.7. Soluble groups and the Geometric classification.}

The basic geometric information that is exploited in this paper is that every soluble connected and simply connected Lie group is topologically homeomorphic to $\mathbb{R}^{d}$ (cf. [1]). Furthermore we shall use the fact that, an essentially unique, left invarient Riemannian structure can be given on any Lie group. Indeed this amounts to assigning, in any way whatsoever, some scalar product on $T_{e}(G)$. The Main Theorem of this paper in Section 0.2 states then:

B) If $Q$ is a soluble simply connected group, then $Q$ is a C-group if and only if it does not have the "polynomial retract property" of Section 0.2.

NB) If $Q$ is above, then it is an NC-group if and only if it does have the "polynomial retract property" of Section 0.2.

If we combine therefore the Main Theorem of this paper with what was said in section OV.5, OV.6, we see that we have obtained the required $\mathrm{B}-\mathrm{NB}$ classification of Lie groups in terms that are:

i) Geometric: The Main Theorem of the present paper. OV.6.

ii) Algebraic: C-NC classification of Lie algebras of sectins OV.3,

iii) Analytic: The behaviour of $\phi(n)$ of Section OV.5.

This is therefore, for general (i.e. not necessarily unimodular) Lie groups, the analogue of the Geometric-Algebraic-Analytic classification of Section OV.4. 


\section{Introduction.}

\subsection{A Classification of Lie algebras.}

Let $\mathfrak{q}$ be some real soluble Lie algebra, we can then choose a basis of $\mathfrak{q}_{c}=\mathfrak{q} \otimes \mathbb{C}$ over $\mathbb{C}$ for which all the ad $x: \mathfrak{q}_{c} \longrightarrow \mathfrak{q}_{c}(x \in \mathfrak{q})$ are represented as upper triangular matrices (cf. [1]). The diagonal coefficients of these matrices are called roots of $\mathfrak{q}$ and can be identified with $\lambda_{1}, \ldots, \lambda_{k} \in \operatorname{Hom}_{\mathbb{R}}[\mathfrak{q}, \mathbb{C}](k=\operatorname{dim} \mathfrak{q})$. We consider then $\left(L_{1}, \ldots, L_{s}\right)=\left(\operatorname{Re} \lambda_{j}, j=1, \ldots, k, \operatorname{Re} \lambda_{j} \neq 0\right) \subset \operatorname{Hom}_{\mathbb{R}}[\mathfrak{q}, \mathbb{R}]=\mathfrak{q}^{*}$ the set of the distinct non-zero real parts of these roots. We say that $\mathfrak{q}$ is a $C$-algebra if there exist $\alpha_{j} \geq 0(j=1, \ldots, k)$ such that

$$
\sum_{j=1}^{s} \alpha_{j} L_{j}=0, \quad \sum \alpha_{j}=1
$$

Otherwise we say that $\mathfrak{q}$ is an $N C$-algebra (Non- $C$-). If $Q$ is some Lie group whose algebra is $C$ (respectively: $N C$ ), we say that $Q$ is $C$ (respectively: $N C$ ). (cf. [2], [3])

Let now $G$ be some simply connected Lie group. It is easy to prove then ( $c f .[4])$ that there exists $Q \subset G$, some simply connected closed soluble subgroup, and $Z \subset G$, some discrete central subgroup, such that

$$
\tilde{Q}=Q \cdot Z \cong Q \times Z \subset G
$$

is closed and co-compact, (i.e. there exists $C \Subset G$ some compact subset such that $\tilde{Q} \cdot C=C \cdot \tilde{Q}=G$. If $G$ is amenable or algebraic we can even take $Z=\{0\})$. We then say that the group $G$, and the corresponding Lie algebra $\mathfrak{g}$, is $B$ - (respectively: $N B$-), if $Q$ is a $C$ - (respectively: $N C$-) group. It is easy to show $(c f .[2])$ that the algebra $\mathfrak{g}$ cannot be simultaneously a $B$ - and an $N B$ - algebra. This last fact is also an easy consequence of our main theorem below.

In my recent work on the area, I have shown that the above $B-N B$ classification is crucial for the behaviour of the Heat kernel of the group. In this paper I shall examine some further consequences in the "global Geometry" of the group. 


\subsection{Riemannian Manifolds.}

The definitions that I shall recall below are variants of notions from [5], [6].

Let $\left(M_{i}, d_{i}\right) i=1,2$ be two metric spaces and let $f: M_{1} \longrightarrow M_{2}$ be some mapping. We set (possibly $+\infty$ )

$$
\|f\|_{\text {Lip }}=\sup \left\{\frac{d_{2}(f(x), f(y))}{d_{1}(x, y)}, x, y \in M, x \neq y\right\}
$$

This is a quasinorm (with $\|f\|=0$ if and only if $f=$ cont.). We say that $f \in \operatorname{Lip}(R)$ if and only if $\|f\|_{\operatorname{Lip}} \leq R$, and we say that $f \in \operatorname{Lip}$ if and only if $f \in \operatorname{Lip}(R)$ for some $R \geq 0$.

We shall consider now $M$ some Riemannian manifold that is topologically homeomorphic with $\mathbb{R}^{n}$. We shall also assume that $M$ is homogeneous, i.e. admits some transitive group of isometrics. And fix some $m_{0} \in M$ and denote by

$$
B(R)=\left\{m \in M: d\left(m, m_{0}\right) \leq R\right\}
$$

the corresponding balls. In our applications $M$ will always be some simply connected soluble real Lie group $Q$ (thus topologically $\cong \mathbb{R}^{n}$, cf. [1]) and $m_{0}=e$ will be the neutral element, and we will assign $Q$ with some left invariant Riemannian structure. There are several such structures, one for each scalar product on the Lie algebra, but they are all quasi-isometric.

The filling constants. We shall consider $f \in \operatorname{Lip}$

$$
f: \partial[0,1]^{n} \longrightarrow M, \quad f(O)=m_{0},
$$

for the boundary and the distance induced on the unit cube $\square^{n}=$ $[0,1]^{n} \subset \mathbb{R}^{n}$ by $\mathbb{R}^{n}$, and the Riemannian distance on $M .(O=(0,0, \ldots) \in$ $\left.\square^{n}\right)$. We shall then define

$$
\phi_{n}(R)=\inf _{f, F}\left\{R^{\prime}\right\}
$$

where $f \in \operatorname{Lip}(R)$ is as in (0.1), and $F: \square^{n} \longrightarrow M$ is such that $\left.F\right|_{\partial \square^{n}}=f$ and $F \in \operatorname{Lip}\left(R^{\prime}\right)$. 
The retract. Let us consider a retract

$$
\begin{gathered}
H: M \times[0,1] \longrightarrow M, \\
H(m, 0)=m_{0}, \quad H(m, 1)=m, \quad m \in M,
\end{gathered}
$$

and let

$$
\psi(R)=\left\|\left.H\right|_{B(R) \times[0,1]}\right\|_{\text {Lip }} .
$$

It is clear that $\phi_{n}(R) \leq C_{n} \psi(R) R(R \geq 1)$. We say that $M$ admits a polynomial (respectively: exponential) retract, if there exist $C, C_{0}>0$ and a retract as above, for which

$$
\psi(R) \leq C R^{C_{0}}+C, \quad R \geq 1 \text { (respectively: } \leq C e^{C_{0} R} \text { ). }
$$

It is an easy matter to show that every soluble Lie group $Q$ as above admits an exponential retract. We have

Main Theorem. Let $Q$ be some simply connected soluble real Lie group. Then:

C) If $Q$ is a $C$-group there exists $2 \leq m \leq \operatorname{rank} Q+1$ such that

$$
\sup _{R>1} \phi_{m}(R) R^{-A}=+\infty, \quad A \geq 1
$$

where $\operatorname{rank} Q=\operatorname{dim} Q / N$ with $N=$ the nilradical of $Q$.

NC) If $Q$ is an NC-group then $Q$ admits a polynomial retract.

The optimal degree of the retract in the $N C$-case (i.e. the $\inf C_{0}$ for $C_{0}$ as in (0.2)) can, in fact, be explicitly computed.

By what has been said, the natural setting of the above theorem is indeed the setting of real simply connected soluble groups, and there is no essential restriction there. To be precise let us call two connected real Lie groups $G_{1}, G_{2}$, Quasiisometric, and denote $G_{1} \underset{q . i}{\simeq} G_{2}$, if there exists a diffeomorphism between $G_{1}$ and $G_{2}$ that is a Riemannian quasiisometry for the corresponding left invariant structures. Let now $G$ be some connected real Lie group that contains no normal compact torus $(\cong$ $\mathbb{T}^{a}, a \geq 1$ ), then we have

$$
U \times K \underset{q . i}{\simeq} G
$$


where $U$ is soluble and simply connected, and $K$ is compact. If $G$ is simply connected this follows from what was said in Section 0.1. The proof in the general case is quite easy also ( $c f .[28])$.

Definition. Let $G$ be some connected Lie group and let $T \subset G$ be its maximal compact normal torus. We then say that $G$ is a geometrically $C$ - (respectively: $N C$-) group, if we can find a quasiisometry as above, such that

$$
U \times K \underset{q . i}{\simeq} G / T
$$

where $U$ satisfies the condition $C$ ) (respectively: $N C)$ ) of the Main Theorem.

It is then an elementary and easy exercise to deduce from the Main Theorem the following:

Geometric Classification Theorem. Let $G$ be some connected real Lie group, then the Lie algebra of $G$ is a $B$ - (respectively: $N B$-) algebra if and only if $G$ is a geometrically $C$ - (respectively: $N C$-) group.

One can also prove that a general connected Lie group $G$ is $N B$ if and only if it has the following:

Homotopy Property. For all $n \geq 1$ there exists $C>1$, such that if $R>0$ and if $F: S^{n} \longrightarrow G$ is a map from the $n$-sphere $S^{n}$ into $G$ that satisfies:

$$
0=[F] \in \pi_{n}(G), \quad F \in \operatorname{Lip}(R)
$$

then there exists a homotopy $H=[0,1] \times S^{n} \longrightarrow G$ such that $H\left(0, S^{n}\right)=$ $g_{0} \in G$ is a fixed point, $\left.H(1, \cdot)\right|_{S^{n}}=F$, and such that $H \in \operatorname{Lip}\left(C R^{C}+\right.$ $C)$.

$[F]$ in $(0.3)$ denotes the homotopy class of $F$ in the $n^{\text {th }}$ Homotopy group of $G$. Indeed the Main Theorem and the fibration $G \longrightarrow G / T \simeq$ $U \times K$ easily reduces the proof of the above assertion to the case where $G$ is compact (and as such an $N B$-group with an abelian $\pi_{1}(G)$ ). The case $G=\mathbb{T}^{a}$ is obvious because the universal covering space $\mathbb{R}^{a} \longrightarrow \mathbb{T}^{a}$ is very simple. The proof for the general case is quite involved and I must confess that at this point I have not written the details down fully. 
This means that unpleasant surprises in a final writting are not to be excluded (especially since my knowledge of Topology is very limited).

The following easy corollary of the Main Theorem is also perhaps worth noting ( $c f$. [8] for a special case).

Corollary. Let $\Gamma$ be a polycyclic group that is a uniform latice in some connected soluble Lie group $Q$ (this can be taken as the definition of a polycyclic group, cf. [7]). Let us assume that $\Gamma$ admits "polynomial filling" in dimensions $2,3, \ldots, \operatorname{dim} Q-1$. (The reader should interpret this "polynomial filling" in terms of the definitions in [5]. There is only one possible such interpretation that is reasonable). Then $\Gamma$ is virtually nilpotent. (The converse is trivially correct).

\subsection{The Homological classification.}

Let $G$ be an arbitrary connected real Lie group and let $|g|=d(g, e)$ $(g \in G)$ be the distance from the neutral element with respect to some fixed left invariant Riemannian structure.

I shall denote by $J(G)$ the space of currents "representable by integration" ( $c f .[13,4.1 .7]$ ) together with the boundary operator $b-$ (or $\partial_{-}$) ( $\left.c f .[12],[13]\right)$. For the reader not familiar with the formalism of currents let me say that $J(G)$ can be identified to the space of differential forms on $G$ with coefficients that are Radon measures. The boundary operator is then identified with the exterior differential taken in the distribution sence. This is simply done by identifying such a form to a linear functional on the space of compactly supported $C^{\infty}$-forms.

For all $\Omega \in J(G)$ and for a fixed left invarient Riemannian structure, if the coefficients of $\Omega$ are $L_{\text {loc }}^{1}$, we can define $|\Omega(x)| \in L_{\text {loc }}^{1}$ the Riemannian norm at almost every $x \in G$, and this can be identified to a Radon measure on $G$ if we specify the reference measure to be the left Haar measure on $G$. By passage to the limit (among other things) $|\Omega(\cdot)|$ can be defined and is a positive Radon measure for all $\Omega \in J(G)$. We can also consider the seminorms

$$
p_{m}(\Omega)=\int_{G}\left(1+|x|^{m}\right) d|\Omega(x)| \leq+\infty, \quad \Omega \in J(G), m \geq 0 .
$$

Dually, let $P(G)$ be the space of differential forms on $G$ with continuous coefficients where the differential $d-$ is taken in the distribution sense 
(i.e.

$$
\int d \omega \wedge \theta= \pm \int \omega \wedge d \theta, \quad \omega \in P(G),
$$

and $\theta$ an arbitrarily compactly supported smooth differential form). Let us then consider the seminorms

$$
q_{m}(\omega)=\sup _{x}|\omega(x)|(1+|x|)^{-m} \leq+\infty, \quad m \geq 0, \omega \in P(G) .
$$

We have the following:

Theorem (The Homological classification). Let $G$ be some real connected Lie group assigned with some left invariant Riemannian structure. Then $G$ is an NB-group if and only if one or both of the following two equivalent conditions hold:

Homology. Let $\Omega \in J(G)$ be such that $\partial \Omega=0$ and $p_{j}(\Omega)<+\infty(j \geq 0)$. Then there exists $\Theta \in J(G)$ such that:

$\partial \Theta-\Omega \in J(G), \quad \operatorname{supp}(\partial \Theta-\Omega)$ is compact, $\quad p_{j}(\Theta)<+\infty, j \geq 0$.

Cohomology. Let $\omega \in P(G)$ be such that $d \omega=0, q_{C}(\omega)<+\infty$ for some $C \geq 0$, then there exists $\theta \in P(G)$ such that

$$
q_{N}(\theta)<+\infty, \quad d \theta-\omega \in E,
$$

where $N \geq 0$ only depends on $G$ and $C$, and where $E \subset P(G)$ is a finite dimensional subspace that only depends on $G$ and satisfies $q_{n}(\lambda)<+\infty$ $(\lambda \in E)$ for some $n=n(G)>0$. Furthermore we can chose $E$ so that it is spanned by a set of representatives of a basis of the cohomology classes of $G$.

The current $\partial \Theta-\Omega$ can even be assumed to be supported in some maximal compact subgroup of $G$. In the critical case of simply connected soluble groups $E$ can be chosen to be the space of constant functions, i.e. the space is then 1-dimensional and so is the unreduced cohomology over $\mathbb{R}$.

The proof of the above Homological classification is implicit in the methods of this paper, it will nonetheless be postponed to a later publication ( $c f$. Remark at the end of Section 4). 


\subsection{The Quadratic filling and further results.}

Some further results will be described in this final subsection in a "sketchy manner". Precise statements and proofs will be given elsewhere.

We shall say that the connected real Lie group admits quadratic filling if for every closed path $\gamma=\varphi\left(\partial \square^{2}\right) \subset G$ that is homotopic to zero in $G$ we can extend $\varphi$ to $\square^{2}$, with $D=\varphi\left(\square^{2}\right)$, so that $\operatorname{Vol}_{2}(D)=$ $O\left(|\gamma|^{2}\right)$, where $|\gamma|=\operatorname{Vol}_{1}(\gamma)$ is the length of the path. (The volumes have to be counted with multiplicity, $c f$. the remark at the end of Section 4.5 below and [29].)

Easy examples of such groups, apart from the Euclidean spaces, are supplied by the semisimple groups (because of the negative curvature of the non compact symmetric spaces), $c f$. [5] for a number of examples that do not admit quadratic filling. Using the standard methods of Morse theory we can also prove that if $G$ does not admit quadratic filling then we can find a sequence $\gamma_{j} \subset G(j \geq 1)$ of periodic geodesics $\operatorname{such}$ that $\operatorname{diam}\left(\gamma_{j}\right) \longrightarrow \infty$.

The above notions generalize to discrete finetely generated groups (cf. [5], or use your imagination). An interesting class of groups that do not admit quadratic filling are the groups with unsolvable word problem. We have also the following analogue of our geodesics on a Lie group:

Let $M$ be some compact connected Riemannian manifold such that $\pi_{1}(M)$ does not admit quadratic filling. Then $M$ contains $\gamma_{j} \subset M$ $(j \geq 1)$ periodic geodesics with prime periods $\left|\gamma_{j}\right| \rightarrow \infty$ (prime period $=$ the time it takes to go round the geodesic once).

So these notions seem to fit in the subject of closed geodesics, $c f$. $[30]$.

\subsection{A Guide to the reader and acknowledgements.}

It is the part $\mathrm{C}$ ) of the main theorem that is difficult. It takes sections 1-4 of this paper to do that. The proof of part NC) relies on easy structure theorems from [1], [2] and is given in Section 5 of this paper.

In Section 1 we develop the necessary algebraic structure theorems for Lie algebras. This part, I feel, presents an independent interest.

Section 2 is routine and reinterprets geometrically the algebraic 
theorems of Section 1 at the group level.

Section 3 was lengthy and tedious to write out, especially at the notational level, and my own inexperience in presenting geometric ideas did not help matters. But there is nothing either difficult or deep in this part. All we do is to exploit the algebraic structure theorems of Section 1 (and their geometric consequences Section 2) to embed some special spheres in a $C$-group $Q$. And that these spheres are "twisted" in such a way that they can not be "filled in" with polynomial estimates.

The denouement lies in Section 4 where the impossibility of that "polynomial filling" is brought into light.

This paper owes a lot to M. Gromov's previous work in the area. Indeed I learned about the problem in [5]. In [5, sections $\left.2 . B, 5 . B_{3}\right]$ one finds a qualitative description in some important examples, of the first geometric construction that I give in sections $3.1,3.2$. In $[5$, sections $\left.5 . B_{1}, 5 . B_{3}\right]$ one finds various proofs of special cases of our main theorem C). These examples were a great inspiration to me.

In fact I feel that one way for the reader to get in this paper, is to pick up the above sections of M. Gromov's [5] and try to see how they fit in the present paper in sections 3.1, 3.2 and 4.5. The reader will then see how to prove the $C$-part of the main theorem for the simplest possible cases of the group semidirect products $\mathbb{R}^{2} \bowtie \mathbb{R}$ and (Heizenberg) $\bowtie \mathbb{R}$ which are the cases contained in [5].

The other point that the reader has to look for, if he wants to capture the global geometric idea of the proof, is to discover the exact role that the $C$-condition plays in the first basic geometric construction. This appears for the first time towards the end of Section 3.2.3 (cf. also the case $\mathbb{R}^{2} \bowtie \mathbb{R} c f$. Section 3.1.1, Remark), and is crucial and nontrivial already in very simple cases like $\mathbb{R}^{3} \bowtie \mathbb{R}^{2}$.

It is difficult to read the proof of the $C$-part of our theorem from beginning to end in a linear fashion. Here are some suggestions of an alternative way to go about it.

1) Read Section 3.1.1 and then 4.5. This will give the special group $D_{2}=\mathbb{R}^{2} \bowtie \mathbb{R}$ with two real non zero roots of opposite sign. This is Gromov's special case and the original reference [5] could also be consulted.

2) Read Section 3.2, where the generalization $D_{r}=\mathbb{R}^{r} \bowtie \mathbb{R}^{r-1}$ is given, and then 4.5. It could be argued that the idea of the construction in $D_{r}$ is also implicit (at least at the topological level) in [5]. We thus have a proof for $D_{r}$. 
The problem now is to embed $D_{r}$ (as a Riemannian submanifold, but not necessarily as a subgroup) in any C-group so as to obtain a general proof.

3) Read Section 1. There we perform the above embedding at the Lie algebra level. This part may not be easy reading but it is just linear algebra and affine geometry and, as such, at least, it is clean. It becomes in particular apparent that the above embedding is not always possible and that we have to consider in addition the groups of rank 1 (cf. 2.4.ii), 3.1.2). In Section 4.5 these groups can be treated exactly as the group $D_{2}$. (cf. also [5]). The reader should check this point.

4) Read Section 2 and assume that the subspace $V_{1}=0$. In a first reading assume also that we are in the split case. Under the above restrictions Section 2.3 simplifies considerably. Then read Section 3.2.4. The assumption that $V_{1}=0$ makes the second basic construction unnecessary (at this point the Remark i) at the end of Section 3.3.1 is relevant). Then use Section 4.5 to finish the proof when $V_{1}=0$ as before.

5) At this point it might be a good idea to study Section 4 where we present a systematic way of how to put things together with the use of the metric properties of current, rather than Transverality and Sard's theorem (from Differential topology). We see, in particular, how the smoothing and the Whitney theorem can be avoided.

6) Read Section 3.3 to be able to deal with the general case $V_{1} \neq 0$. In doing so, in a first reading, the reader should absolutely start with the split case, which is simpler and yet already contains the main idea of the construction. It is here that a good understanding of Section 2 (first for the split case and then for the general case) is essential.

Convention. I use throughout the convention that, in a formula, the letters $C$ or $c$, possibly with suffixes, indicate, possibly different, positive constants that are independent of the important parameters of the formula. 


\section{Algebra and Combinatorics.}

\subsection{Combinatorial considerations.}

\subsubsection{Simplexes.}

Let $V$ be some finite dimensional real vector space and let $E=$ $\left(e_{1}, \ldots, e_{k}\right) \subset V$ be a finite subset where the $e_{j}$ 's are assumed to be distinct. We shall denote

$$
C H(E)=\text { Convex Hull }(E)=\left\{\sum_{j=1}^{k} \lambda_{j} e_{j}: \lambda_{j} \geq 0 \quad \sum \lambda_{j}=1\right\} .
$$

If the topological dimension of $C H(E)$ is $k-1$ we shall say that $E$ are the vertices of a simplex and denote

$$
\begin{gathered}
\sigma=[E]=C H(E)=\text { simplex spanned by } E, \\
\operatorname{Int} \sigma=\left\{\sum_{j=1}^{k} \lambda_{j} e_{j}: \lambda_{j}>0, \sum \lambda_{j}=1\right\} .
\end{gathered}
$$

Int $\sigma$ is not to be confused with $\stackrel{\circ}{\sigma} \subset \sigma$ the topological interior of $\sigma \subset V$. We say that $\sigma$ is not degenerate if $\operatorname{Int} \sigma=\stackrel{\circ}{\sigma}$, i.e. if and only if $k=$ $\operatorname{dim} V+1$.

Let $\sigma=\left[x_{0}, x_{1}, \ldots, x_{k}\right] \subset \mathbb{R}^{k}$ be some simplex and let $A_{0}$ be the affine hyperplane containing the face $\left[x_{1}, \ldots, x_{k}\right] \subset \sigma$. Let us assume that

$$
\begin{aligned}
0 \notin A_{0} & =\left\{\sum_{j=1}^{k} \lambda_{j} x_{j}: \sum \lambda_{j}=1\right\} \\
& =x_{1}+\operatorname{Vec}\left(x_{j}-x_{1}, 1 \leq j \leq k\right) .
\end{aligned}
$$

If we assume $\sigma$ to be nondegenerate, the vectors $x_{j}-x_{1} \in V(2 \leq j \leq k)$ are linearly independent and $\operatorname{dim} V=k$. (1.1.1) implies then that $x_{1}, x_{2}, \ldots, x_{k}$ is a basis of $V$.

Let us also recall the general fact that if $x \in C H(E) \subset V$ then we can choose $E^{\prime} \subset E$ such that

$$
x \in C H\left(E^{\prime}\right), \quad \operatorname{Card}\left(E^{\prime}\right) \leq \operatorname{dim} V+1 .
$$


Indeed we can assume without loss of generality that $E$ are the extreme points of some convex polyhedron $P \subset V$ and that $x \in \stackrel{\circ}{P}$.

Let $e_{1} \in E$ and let

$$
y=\partial P \cap\left\{\text { affine line through } e_{1} \text { and } x\right\} .
$$

$y$ then lies in some boundary convex polyhedron of lower dimension. This by induction on $\operatorname{dim} V$ proves our assertion.

We shall adopt the standard notation of covering with a "^" any symbol that we want to delete. We have then

Lemma. Let $P=C H\left(p_{1}, \ldots, p_{n}\right) \subset V=\mathbb{R}^{k}$ be some convex polyhedron with non-empty interior: $\stackrel{\circ}{P} \neq \varnothing$. Let us assume that $P$ is not a simplex and let

$$
P_{j}=C H\left(p_{1}, p_{2}, \ldots, \hat{p}_{j}, \ldots, p_{n}\right), \quad j=1, \ldots, n
$$

Then

$$
P=\cup\left[P_{j} ; j=1, \cdots, n, \stackrel{\circ}{P}_{j} \neq \varnothing\right] .
$$

Proof. Let $\Sigma_{1}, \Sigma_{2}, \ldots$ be the finitely many convex polyhedra that we obtain by

$$
\Sigma_{J}=C H\left(p_{j}, j \in J\right), \quad J \subset(1, \ldots, n), \quad|J|=k+1 .
$$

Let $x \in P$. By (1.1.2) it follows that one of the above polyhedra, say $\Sigma_{1}$, has positive Lebesgue density at $x$ and therefore

$$
x \in \Sigma_{1}, \quad \stackrel{\circ}{\Sigma}_{1} \neq \varnothing,
$$

and, since by our hypothesis $n>k+1$, there exists $1 \leq j \leq n$ such that $P_{j} \supset \Sigma_{1}$. This proves the Lemma.

\subsubsection{The abstract $\mathcal{A}$-condition.}

Let $V$ be some finite dimensional vector space over $\mathbb{R}$, and let us decompose the class of all finite subsets $E \subset V$ into two classes, $\mathcal{A}$ and $\sim \mathcal{A}$ (i.e. $\mathcal{A}$ is some property that $E$ may or may not have). We shall suppose that 
i) $\varnothing \notin \mathcal{A}$,

ii) $E \subset V, E \in \mathcal{A}$ if and only if $E \backslash\{0\} \in \mathcal{A}$,

iii) $E_{1} \subset E \subset V, E_{1} \in \mathcal{A}$ implies $E \in \mathcal{A}$,

iv) $E_{1} \subset E \subset V, C C(E)=C C\left(E_{1}\right), E \in \mathcal{A}$ implies $E_{1} \in \mathcal{A}$,

where

$$
C C(X)=\text { Convex Cone }(X)=C H(\lambda X, \lambda \geq 0), \quad X \subset V,
$$

ii) and iv) say that we can delete from some $E \in \mathcal{A}$ any "positive" linear combination of the remaining elements without spoiling the property $\mathcal{A}$.

It is clear that if $\mathcal{A}$ and $\mathcal{A}^{\prime}$ are two such properties, the property $\mathcal{A} \cap \mathcal{A}^{\prime}$ also satisfies the same conditions. In the following sections we shall deal with the following special cases: $V \neq\{0\}$ and

$$
\begin{aligned}
& E \in \mathcal{A}_{1} \text { if and only if } E \text { spans } V \\
& E \in \mathcal{A}_{2} \text { if and only if } 0 \in C H(E \backslash\{0\}) .
\end{aligned}
$$

If and only if $E \in \mathcal{A}_{2}$ we say that $E$ satisfies the $C$ - condition. If and only if $E \notin \mathcal{A}_{2}$ we say that it is $N C$.

\subsubsection{Minimal $\mathcal{A}$-sets.}

We say that $E \subset V$ is a minimal $\mathcal{A}$-set if

$$
E \in \mathcal{A}, E \backslash\{e\} \notin \mathcal{A}, \quad \text { for all } 0 \neq e \in E .
$$

If $\mathcal{A}=\mathcal{A}_{1}$ then clearly $E$ is a minimal $\mathcal{A}_{1}$ set if $E \backslash\{0\}$ is a basis of $V$. In this section we shall examine the minimal $\mathcal{A}$ sets with $\mathcal{A}=\mathcal{A}_{2}$, $\mathcal{A}_{1} \cap \mathcal{A}_{2}$.

By definition, $E \subset V$ is a minimal $\mathcal{A}_{2}$ set if and only if the following two conditions hold

$$
\begin{gathered}
0=\sum_{e \in E \backslash\{0\}} \lambda_{e} e, \quad \lambda_{e}>0, \\
0=\sum_{e \in E \backslash\{0\}} \mu_{e} e, \quad \mu_{e} \geq 0, \quad \sum \mu_{e}=1 \\
\text { implies } \mu_{e}>0, e \in E \backslash\{0\} .
\end{gathered}
$$


Let us consider

$$
0=\sum_{e \in E \backslash\{0\}} \nu_{e} e, \quad \nu_{e} \in \mathbb{R}
$$

there exists $e \in E \backslash\{0\}$ such that $\nu_{e} \neq 0$.

Then by rescaling the $e_{j}$ 's (i.e. replacing $e_{j}$ by $e_{j}^{\prime}=\varepsilon_{j} e_{j}, \varepsilon_{j}>0$ ), by giving an appropriate order to $E \backslash\{0\}$, and by multiplying (R) and (C) by scalars we can assume that

$$
1=\lambda_{1} \leq \lambda_{2} \leq \cdots
$$

we can assume that there exists $\lambda>0$ such that

$$
\left|\nu_{j}\right|=0, \lambda, \quad j=1,2, \ldots
$$

and we can also assume that for the first $j=1,2, \ldots$ for which $\nu_{j} \neq 0$ we have $\nu_{j}=-\lambda_{j}$.

But then the relation $(\mathrm{R})+(\mathrm{C})$ is a positive relation on $E \backslash\{0\}$ of length strictly less than $|E \backslash\{0\}|$. This by (1.1.3) implies that $(\mathrm{R}) \equiv-$ (C). In other words, (C) is up to multiplicative constant the only linear relation on $E \backslash\{0\}$. It follows therefore that $E \subset V$ is a minimal $\mathcal{A}_{2}$ set if and only if $E$ are the vertices of some simplex of $V$ and

$$
0 \in \operatorname{Int}[E]
$$

Let now $E \subset V$ and

$$
X=E \backslash\{0\}=\left(x_{1}, \ldots, x_{n}\right) \subset V .
$$

Let also $X_{j}=X \backslash\left\{x_{j}\right\}$. Then by definition, $E$ is a minimal $\mathcal{A}_{1} \cap \mathcal{A}_{2}$ set if and only if:

a) Vec $E=V$.

b) There exists $\alpha_{j} \geq 0, j=1, \ldots, n$, such that $\sum_{j=1}^{n} \alpha_{j}=1$, $\sum_{j=1}^{n} \alpha_{j} x_{j}=0$. hold:

c) For $k=1,2, \ldots, n$ one of the conditions i) or ii) below (or both)

i) $X_{k}$ is $N C$.

ii) $\operatorname{Vec} X_{k} \neq V$. 
We have:

Proposition. Let $E \subset V$ be some minimal $\mathcal{A}_{1} \cap \mathcal{A}_{2}$ set. Then $\sigma=$ $[E \backslash\{0\}]$ is a non-degenerate simplex and $0 \in \sigma$.

Proof. By b), by reordering if necessary the set $X$, we have:

$$
-x_{1} \in \Omega=C C\left(x_{2}, \ldots, x_{n}\right) .
$$

But this together with a) implies that

$$
\operatorname{Vec}\left(X_{1}\right)=V \text {, }
$$

which together with c) implies that $X_{1} \subset V$ is an $N C$-set. It follows that if we slice $\Omega$ by some appropriate affine hyperplane $0 \notin H \subset V$ the convex polyhedron

$$
\begin{gathered}
P=H \cap \Omega=C H\left(p_{2}, \ldots, p_{n}\right) \subset H, \\
p_{j}=\left[\lambda x_{j}, \lambda>0\right] \cap H, \quad j=2, \ldots, n,
\end{gathered}
$$

will satisfy $\varnothing \neq \stackrel{\circ}{P} \subset H$. Let

$$
\Omega_{k}=C C\left(\hat{x}_{1}, x_{2}, \ldots, \hat{x}_{k}, \ldots, x_{n}\right), \quad P_{k}=\Omega_{k} \cap H \subset P \subset H .
$$

Clearly, for each $k=2, \ldots, n$, the relative interior of $P_{k}$ is non empty $\left(\varnothing \neq \stackrel{\circ}{P}_{k} \subset H\right)$ if and only if

$$
\operatorname{Vec}\left(X \backslash\left\{x_{1}, x_{k}\right\}\right)=V .
$$

If for some $k=2, \ldots, n(1.1 .5)$ holds, we must have

$$
x_{1} \notin-\Omega_{k} .
$$

Indeed, if not and $x_{1} \in-\Omega_{k}$, the set $X_{k}$ satisfies the $C$-condition, but this together with (1.1.5) contradicts $\mathrm{c}$ ).

If we combine (1.1.4), (1.1.5) and (1.1.6), we see that

$$
P \neq \cup\left[P_{k} ; k=2, \ldots, n, \stackrel{\circ}{P}_{k} \neq \varnothing\right] .
$$

It follows by the Lemma in 1.1.1 that $P$ is a non degenerate simplex. And from the choice of $H$ and the remark at the beginning of Section 1.1.1 
it follows that $x_{2}, x_{3}, \ldots, x_{n}$ is a basis of $V$. This together with (1.1.4) completes the proof of our proposition. To see how this is done, we can assume without loss of generality that $x_{2}, \ldots, x_{n}$ are the coordinate unit vectors $\mathbb{I}_{j}=(0,0, \ldots, 1,0, \ldots, 0) ;(1.1 .4)$ simply says then that $x_{1}$ lies in the negative quadrant. $\sigma=\left[x_{1}, \mathbb{I}_{j}, j=1, \ldots, n-1\right]$ is then clearly a non degenerate simplex and $0 \in \sigma$.

REMARKS.

i) One should observe that we can reformulate the above proposition and say: $E \subset V$ is a minimal $\mathcal{A}_{1} \cap \mathcal{A}_{2}$ if and only if there exists $V=V_{1} \oplus V_{2}$ a direct decomposition of the space such that

$$
E=\left(E \cap V_{1}\right) \cup\left(E \cap V_{2}\right)=E_{1} \cup E_{2}, \quad V_{2} \neq\{0\},
$$

and:

a) Either $V_{1}=\{0\}$ or $E_{1} \backslash\{0\}$ is a basis of $V_{1}$.

b) $\sigma=\left[E_{2} \backslash\{0\}\right]$ is a non-degenerate simplex in $V_{2}$ and $0 \in \operatorname{Int} \sigma$.

ii) It is an interesting exercise (but of no use to us) to work out the minimal $\mathcal{A}_{3}$ sets where $E \in \mathcal{A}_{3}$ if and only if $C H(E \backslash\{0\})=C \subset V$ contains 0 in its interior $0 \in \stackrel{\circ}{C}$. Such a set need not necessarily be a simplex.

\subsubsection{The minimal $\mathcal{A}$-couple.}

Let $V$ be some finite dimensional vector space and let $A \subset E \subset V$ be two finite subsets. We shall say that $A \subset E$ is a minimal $\mathcal{A}$-couple if

i) $A \in \mathcal{A}$.

ii) $E \subset A+A+\cdots=\left\{\alpha_{1}+\alpha_{2}+\cdots+\alpha_{p} ; \alpha_{j} \in A, j=1, \ldots, p\right.$, $p \geq 1\}$.

iii) $0 \neq \alpha \in A$ implies $E \backslash\{\alpha\} \notin \mathcal{A}$.

It is clear that then $A$ is a minimal $\mathcal{A}$-set. Note also that because of ii) we can replace i) by:

i) $)^{\prime} E \in \mathcal{A}$. 
Example. $\mathcal{A}=\mathcal{A}_{1}$. It is then clear that $A \subset E$ a minimal couple if and only if $A \backslash\{0\}$ is a basis of $V$ and

$$
B=E \backslash A \subset\{0\}, \quad \text { i.e. } B=\{0\}, \varnothing .
$$

Let now $A \subset E$ be some minimal $\mathcal{A}_{2}$ couple, then by 1.1.3 the points of $A$ are the vertices of some simplex

$$
\sigma=[A]=\left[\alpha_{1}, \ldots, \alpha_{k}\right], \quad 0 \in \operatorname{Int} \sigma .
$$

Let

$$
\sigma_{j}=\left[0, \alpha_{1}, \ldots, \hat{\alpha}_{j}, \ldots, \alpha_{k}\right], \quad j=1,2, \ldots, k,
$$

be the simplex that we obtain by replacing $\alpha_{j}$ by $0(1 \leq j \leq k)$.

It is clear that

$$
\bigcup_{j} \sigma_{j}, \quad \bigcup_{j}\left(-\sigma_{j}\right) \subset V^{\prime}=\operatorname{Vec}\left(\alpha_{1}, \ldots, \alpha_{k}\right) \subset V,
$$

are neighbourhoods of 0 in $V^{\prime}$. The condition iii) (and the definition of the $C$-condition) implies on the other hand that if $B \neq \varnothing$, then

$$
-\sigma_{j} \cap C C(B)=\{0\}, \quad j=1,2, \ldots, k .
$$

(1.1.8), (1.1.9) together imply that

$$
V^{\prime} \cap C C(B)=\{0\} .
$$

Therefore (1.1.7) holds again. We have:

Proposition. Let $A \subset E \subset V$ be some $\mathcal{A}_{1} \cap \mathcal{A}_{2}$ minimal couple. Then

$$
B=E \backslash A=\varnothing,\{0\} .
$$

Furthermore there exists some direct decomposition $V=V_{1} \oplus V_{2}$ with $V_{2} \neq\{0\}$ such that

$$
\begin{gathered}
A=\left(A \cap V_{1}\right) \cup\left(A \cap V_{2}\right)=A_{1} \cup A_{2}, \\
\text { either } V_{1}=\{0\} \text { or } A_{1} \backslash\{0\} \text { is a basis of } V_{1}, \\
A_{2} \backslash\{0\} \text { are the vertices of some } \\
\text { non-degenerate simplex in } V_{2},
\end{gathered}
$$


and

$$
0 \in \operatorname{Int}\left[A_{2} \backslash\{0\}\right]
$$

Proof. Let

$$
\begin{aligned}
A \backslash\{0\}=\left(\alpha_{1}, \ldots, \alpha_{n}\right), & \sigma=C H(A \backslash\{0\}), \\
V^{i}=\operatorname{Vec}\left(\alpha_{1}, \ldots, \hat{\alpha}_{i}, \ldots, \alpha_{n}\right), & \sigma_{i}=\left[\alpha_{1}, \ldots, \hat{\alpha}_{i}, \ldots, \alpha_{n}\right] .
\end{aligned}
$$

By the proposition in 1.1.3 and the fact that $A$ is a minimal $\mathcal{A}_{1} \cap \mathcal{A}_{2}$ set it follows that

$$
0 \in \sigma, \quad \sigma \text { is a non-degenerate simplex } \subset V .
$$

This in turn implies that

(1.1.15) $\operatorname{dim} V^{i} \geq \operatorname{dim} \operatorname{Vec}(A)-1=\operatorname{dim} V-1, \quad i=1,2, \ldots, n$.

We can distinguish two cases:

Case i) $0 \in \stackrel{\circ}{\sigma}=\operatorname{Int} \sigma$.

Case ii) There exists $1 \leq m<n$ such that

$$
0 \in \sigma_{j}, 1 \leq j \leq m, \quad 0 \notin \sigma_{j}, m<j \leq n,
$$

where we suppose that we have, if necessary, reordered the set $\left(\alpha_{1}, \ldots\right.$, $\left.\alpha_{n}\right)$.

In case i) our proposition follows by repeating verbatim the proof of (1.1.7) for the previous case $\mathcal{A}=\mathcal{A}_{1}$. We shall assume therefore that we are in case ii). We then claim that

$$
B \subset V^{i}, \quad i=1,2, \ldots, m .
$$

Indeed, if not, there exists

$$
\operatorname{Vec}(A) \ni \beta \notin V^{i} \text {, for some, say } i=1,1 \leq i \leq m \text {. }
$$

But then (1.1.15) implies that

$$
\operatorname{Vec}\left(E \backslash\left\{\alpha_{1}\right\}\right)=V \text {. }
$$


On the other hand, since $0 \in \sigma_{1}$ the set $A \backslash\left\{0, \alpha_{1}\right\}$ and, a fortiori, the set $E \backslash\left\{\alpha_{1}\right\}$ satisfies the $C$-condition and $E \backslash\left\{\alpha_{1}\right\} \in \mathcal{A}_{1} \cap \mathcal{A}_{2}$ in contradiction with iii). This proves (1.1.16).

We shall set now

$$
\begin{gathered}
V_{1}=\operatorname{Vec}\left(\alpha_{1}, \ldots, \alpha_{m}\right), \quad V_{2}=\operatorname{Vec}\left(\alpha_{m+1}, \ldots, \alpha_{n}\right), \\
\tilde{\sigma}=\left[\alpha_{m+1}, \ldots, \alpha_{n}\right] .
\end{gathered}
$$

It follows by the conditions of case ii) that $\alpha_{1}, \ldots, \alpha_{m}$ is a basis of $V_{1}$ and $\tilde{\sigma}$ is a non-degenerate simplex of $V_{2}$ such that

$$
0 \in \operatorname{Int} \tilde{\sigma}, \quad \tilde{\sigma}=\bigcap_{j=1}^{m} \sigma_{j} .
$$

Observe that if $A=$ affine subspace spaned by $\alpha_{1}, \ldots \alpha_{m}$, then $\widetilde{\sigma} \cap A=$ $\varnothing$ and therefore $0 \notin A$, then use the argument of (1.1.1).

This proves the conditions (1.1.11), (1.1.12), (1.1.13) and (1.1.14) of the proposition and that $V=V_{1} \oplus V_{2}$. It follows from (1.1.16) that $B \subset \bigcap_{i=1}^{m} V^{i}=V_{2}$. The condition (1.1.10) follows because what we have shown implies that

$$
\left(A \cap V_{2}\right) \subset\left(E \cap V_{2}\right) \subset V_{2}
$$

is a minimal couple in $V_{2}$ that falls under our previous case i).

This completes the proof of the proposition.

\subsubsection{Inner product spaces.}

We shall now assume that the vector space $V$ is assigned with an inner product $\langle\cdot, \cdot\rangle$. Let then $E=\left(e_{1}, \ldots, e_{n}\right) \subset V$ be some NC set $(c f$. 1.1.2) such that $e_{j} \neq 0,1 \leq j \leq n$. By Hahn-Banach this is equivalent to the fact that there exists $u \in V$ such that

$$
\left\langle u, e_{j}\right\rangle>0, \quad 1 \leq j \leq n .
$$

We shall show that it is possible to choose the $u$ in (1.1.17) to satisfy in addition the condition

$$
u \in C H(E) .
$$


Indeed let $u \in V$ be as in (1.1.17) and let

$$
e_{j}=\left\langle u, e_{j}\right\rangle u+e_{j}^{\prime}, \quad\left\langle u, e_{j}^{\prime}\right\rangle=0, \quad j=1, \ldots, n .
$$

We can now distinguish a number of cases:

1) $u=\lambda e_{j}$ for some $\lambda>0,1 \leq j \leq n$. Then (1.1.18) holds.

2) The set $E^{\prime}=\left(e_{1}^{\prime}, \ldots, e_{n}^{\prime}\right)$ is $\mathrm{NC}$ and $e_{j}^{\prime} \neq 0,1 \leq j \leq n$. By induction on the dimension of $V$ there exist then

$$
u^{\prime}=\Sigma \alpha_{j} e_{j}^{\prime}, \Sigma \alpha_{j}=1, \alpha_{j} \geq 0,\left\langle e_{j}^{\prime}, u^{\prime}\right\rangle>0,1 \leq j \leq n
$$

Then $u_{1}=\Sigma \alpha_{j} e_{j}$ satisfies (1.1.17), (1.1.18).

3) There exist $\alpha_{j} \geq 0, \Sigma \alpha_{j}=1$ such that $\Sigma \alpha_{j} e_{j}^{\prime}=0$. But then

$$
u_{1}=\Sigma \alpha_{j} e_{j}=\left(\Sigma \alpha_{j}\left\langle u, e_{j}\right\rangle\right) u
$$

satisfies (1.1.17), (1.1.18).

By a slight perturbation, we can even guarantee that the $u \in V$ that satisfies (1.1.17) and (1.1.18) is of the form

$$
u=\sum_{j=1}^{n} \lambda_{j} e_{j}, \quad \lambda_{j}>0,1 \leq j \leq n .
$$

\subsection{Algebraic considerations.}

In this section, we shall recall some standard facts and definitions and also introduce some new notions. All the Lie algebras in this section, unless otherwise stated, will be finite dimensional and defined over $\mathbb{R}$.

i) Subnormal subalgebras. Let $\mathfrak{g}_{1} \subset \mathfrak{g}$ be a Lie algebra and a subalgebra. We say that $\mathfrak{g}_{1}$ is a subnormal subalgebra and denote $\mathfrak{g}_{1} \triangleleft \triangleleft \mathfrak{g}$ if there exist subalgebras

$$
\mathfrak{g}_{1} \subset \mathfrak{g}_{2} \subset \cdots \subset \mathfrak{g}_{p}=\mathfrak{g}, \quad p \geq 1
$$

such that $\mathfrak{g}_{j} \triangleleft \mathfrak{g}_{j+1}$ (i.e. $\mathfrak{g}_{j}$ is an ideal of $\mathfrak{g}_{j+1}$ ) for $j=1, \ldots, p-1$. 
If $\mathfrak{g}$ is assumed soluble it follows (without extra cost) that we can assume that $\operatorname{dim}\left(\mathfrak{g}_{j+1} / \mathfrak{g}_{j}\right)=1,1 \leq j \leq p-1$. It is clear that the above relation is transitive, i.e.

$$
\mathfrak{g}_{1} \triangleleft \triangleleft \mathfrak{g}_{2} \triangleleft \triangleleft \mathfrak{g}_{3} \text { implies } \mathfrak{g}_{1} \triangleleft \triangleleft \mathfrak{g}_{3} \text {. }
$$

Quite generally for any Lie algebra $\mathfrak{q}$, we shall denote by $\mathfrak{z}(\mathfrak{q})$ it center. Observe also that if $\mathfrak{a} \subset \mathfrak{n}$ is any subalgebra of the nilpotent algebra $\mathfrak{n}$, then $\mathfrak{a}$ is subnormal. To see that one has to distinguish the two cases

$$
\mathfrak{z}(\mathfrak{n}) \subset \mathfrak{a}, \quad \mathfrak{z}(\mathfrak{n}) \not \subset \mathfrak{a}
$$

and use induction.

ii) Nilpotent $\mathfrak{g}$-algebras. Let $\mathfrak{n}$ be some nilpotent Lie algebra and let $\mathfrak{g} \subset \partial(\mathfrak{n})$ be some Lie subalgebra of the Lie algebra of derivations of $\mathfrak{n}$ (i.e. $\mathfrak{g}$ acts on $\mathfrak{n}$ by derivations). We shall then denote by $[x, y]=$ $-[y, x] \in \mathfrak{n}, x \in \mathfrak{g}, y \in \mathfrak{n}$ the action of $\mathfrak{g}$ on $\mathfrak{n}$ and consider $\mathfrak{n}_{1} \subset \mathfrak{n}$ the $\mathfrak{g}$ subalgebras of $\mathfrak{n}$, i.e. the subalgebras for which $\left[\mathfrak{g}, \mathfrak{n}_{1}\right] \subset \mathfrak{n}_{1}$.

iii) Abstract root algebras. Let $\mathfrak{n}$ be some nilpotent $\mathfrak{g}$ algebra $(\mathfrak{g} \subset$ $\partial(\mathfrak{n}))$ as above. Let $V$ be some finite dimensional vector space which we shall call the space of roots. Let $E \subset V$ be a finite subset of elements which will be called roots. For every $e \in E$ we shall consider $\mathfrak{n}_{e} \subset \mathfrak{n}$ a subspace which we shall call the root space of $e \in E$. We shall say that $\mathfrak{n}, \mathfrak{g}, V, E, \mathfrak{n}_{e}$ (or simply $\mathfrak{n}$ or $\mathfrak{n}, \mathfrak{g}$ ), are an abstract root algebra if the following conditions are verified:

a) $\left[\mathfrak{n}_{e}, \mathfrak{g}\right] \subset \mathfrak{n}_{e} \neq\{0\}, e \in E$.

b) For $e_{1}, e_{2} \in E$

$$
\left[\mathfrak{n}_{e_{1}}, \mathfrak{n}_{e_{2}}\right] \subset \begin{cases}\{0\}, & \text { if } e_{1}+e_{2} \notin E, \\ \mathfrak{n}_{e_{1}+e_{2}}, & \text { if } e_{1}+e_{2} \in E\end{cases}
$$

c) For every $\mathfrak{g}$-subalgebra $\tilde{\mathfrak{n}} \subset \mathfrak{n}$ (and in particular for $\tilde{\mathfrak{n}}=\mathfrak{n}$ ) we have a direct sum decomposition

$$
\tilde{\mathfrak{n}}=\bigoplus_{e \in E}\left(\tilde{\mathfrak{n}} \cap \mathfrak{n}_{e}\right)
$$

The trivial case $\mathfrak{n}=\{0\}, E=\varnothing$ shall, for convenience, be admitted in the above definition. 
It is clear that if $\left(\mathfrak{n}, \mathfrak{g}, V, E, \mathfrak{n}_{e}\right)$ is a root algebra and $\tilde{V} \supset V$ then $\left(\mathfrak{n}, \mathfrak{g}, \tilde{V}, E, \mathfrak{n}_{e}\right)$ is also a root algebra in a natural way. And if $\tilde{\mathfrak{n}} \subset \mathfrak{n}$ is $\mathfrak{g}$-subalgebra of $\mathfrak{n}$ then $\left(\tilde{\mathfrak{n}}, \mathfrak{g}, V, \tilde{E}, \tilde{\mathfrak{n}}_{e}=\mathfrak{n}_{e} \cap \tilde{\mathfrak{n}}\right)$ is also a root algebra with

$$
\tilde{E}=\left\{e \in E, \tilde{\mathfrak{n}} \cap \mathfrak{n}_{e} \neq\{0\}\right\} .
$$

If a property $\mathcal{A}$ has been assigned on the finite subsets of $V$ as in Section 1.1.2, we shall say that the root algebra $\left(\mathfrak{n}, \mathfrak{g}, V, E, \mathfrak{n}_{e}\right)$ is an $\mathcal{A}$-algebra if $E \in \mathcal{A}$.

EXAMPLES.

iv) The Zassenhaus decomposition. Let $\mathfrak{n}$ be some nilpotent complex algebra and let us assume that $\mathfrak{g}$ is also nilpotent. We can consider then

$$
\mathfrak{n}=\bigoplus_{e} \mathfrak{n}_{e}, \quad e \in E \subset \operatorname{Hom}_{\mathbb{R}}[\mathfrak{g}, \mathbb{C}]
$$

where $e$ are the roots of the Zassenhaus root space decomposition of the $\mathfrak{g}$ action on the complex vector space $\mathfrak{n}$. We obtain thus a root algebra (cf. [9]).

The nilpotency of $\mathfrak{g}$ is essential for the above to work for otherwise we do not have root space decomposition. Even in the case when $\mathfrak{n}$ is abelian, i.e. is just a complex vector space, and $\mathfrak{g}$ is soluble, where we can define the roots of the action - by Lie's theorem $c f$. [9] -, we cannot in general define root spaces.

v) The real root space Zassenhaus decomposition. The following modification of the above example is a forerunner of things to come. $\mathfrak{n}$ is a real nilpotent algebra and $\mathfrak{g}$ is nilpotent. We have then the corresponding Zassenhaus decomposition

$$
\mathfrak{n} \otimes \mathbb{C}=\bigoplus_{e} \mathfrak{n}_{e}, \quad e \in E
$$

We can write then $e=\operatorname{Re} e+i \operatorname{Im} e$ where $\operatorname{Re} e, \operatorname{Im} e \in \mathfrak{g}^{*}=\operatorname{Hom}_{\mathbb{R}}[\mathfrak{g}, \mathbb{R}]$. It is then very easy to see that

$$
\bigoplus_{\substack{e \in E \\ \operatorname{Re} e=L}} \mathfrak{n}_{e}=\mathfrak{n}_{L}=\tilde{\mathfrak{n}}_{L} \otimes \mathbb{C}, \quad L \in \mathfrak{g}^{*}
$$


where I use the notations of [2, Section 1.2], and where $\tilde{\mathfrak{n}}_{L} \subset \mathfrak{n}$. When $\tilde{\mathfrak{n}}_{L} \neq 0$ we call this the real root space with real root $L \in \mathfrak{g}^{*}$, and we have the corresponding "real root space decomposition"

$$
\mathfrak{n}=\bigoplus_{L \in \Lambda} \tilde{\mathfrak{n}}_{L}, \quad \Lambda=\{\operatorname{Re} e, e \in E\} \subset \mathfrak{g}^{*},
$$

the elements of $\Lambda$ will be called the "real roots". This gives again an abstract root algebra in the sense of iii).

vi) The basic example of a soluble Lie algebra. The set up will be the same as in [2, sections 1.1-1.3] the notations there will be preserved: $\mathfrak{q} \supset \mathfrak{n}$ is a soluble real Lie algebra together with its nilradical, $\mathfrak{h}$ will be some nilpotent almost complement of $\mathfrak{n}$ (e.g. a Cartan subalgebra - so that $[2,(1.1 .9)] \mathfrak{q}=\mathfrak{n}+\mathfrak{h})$. We have then

$$
\mathfrak{n}=\mathfrak{n}_{0} \oplus \mathfrak{n}_{1} \oplus \cdots \oplus \mathfrak{n}_{k}
$$

the real root space decomposition of $[2,(1.3 .4)]$ where $\mathfrak{n}_{0}$ corresponds to the real root 0 , and abusively $\mathfrak{n}_{0}$ could be $\mathfrak{n}_{0}=\{0\}$. We shall set $\mathfrak{g}=\mathfrak{n}_{0}+\mathfrak{h}$ (equal to $\mathfrak{q}_{R}$ with the notations of the proposition of $[2,1.3]$ ) which is now a soluble algebra (but not in general nilpotent).

The above set up gives us an abstract root algebra $(\mathfrak{n}, \mathfrak{g})$ where the root space decomposition is given by (1.2.1). The set of the roots $E$ can be identified to a subset of any one of the following spaces $\mathfrak{q}^{*}, \mathfrak{g}^{*},\left(\mathfrak{g} / \mathfrak{n}_{0}\right)^{*}=\left(\mathfrak{h} / \mathfrak{h} \cap \mathfrak{n}_{0}\right)^{*}=(\mathfrak{q} / \mathfrak{n})^{*}$ by the obvious identifications. Any of these spaces could thus be taken as the space of roots.

vii) Subalgebra of the abelian and Heizenberg type. In this section $\mathfrak{n}$ is a general root $\mathfrak{g}$-algebra.

vii) $)_{a}$ Let $\mathfrak{a} \subset \mathfrak{n}$ be an abelian $\mathfrak{g}$-subalgebra of $\mathfrak{n}$. We have then

$$
\mathfrak{a}=\bigoplus_{i=0}^{k} \mathfrak{a}_{i}, \quad \mathfrak{a}_{i}=\mathfrak{n}_{i} \cap \mathfrak{a}, \quad i=0, \ldots, k
$$

where, for convenience, I use the notations of (1.2.1). If we erase the zero components we obtain the corresponding root space decomposition of that subalgebra.

vii $_{h}$ We shall also consider subalgebras of $\mathfrak{n}$ of Heizenberg type. This is what we mean: 
We shall assume that

$$
\alpha_{1},-\alpha_{1}, \alpha_{2},-\alpha_{2}, \ldots, \alpha_{p},-\alpha_{p} \subset E
$$

are non-zero distinct roots among the roots of $\mathfrak{n}$, and we shall assume that

$$
\{0\} \neq H_{ \pm j} \subset \mathfrak{n}_{ \pm \alpha_{j}}, \quad j=1,2, \ldots, p
$$

are non-trivial $\mathfrak{g}$-subspaces such that among all the brackets,

$$
\left.\left[x_{1},\left[x_{2}, \ldots\right], x_{k}\right], \ldots\right], \quad x_{j} \in H_{ \pm}=\left\{\sum H_{i}, i \neq 0,-p \leq i \leq p\right\}
$$

where $1 \leq j \leq k$, the only ones that may not be zero come from

$$
\left[H_{i}, H_{-i}\right], \quad i=1,2, \ldots, p \text {. }
$$

It is clear then that

$$
H=H_{ \pm} \oplus H_{0}=H_{ \pm} \oplus \sum_{i=1}^{p}\left[H_{i}, H_{-i}\right]
$$

is a $\mathfrak{g}$-subalgebra of $\mathfrak{n}$ and that

$$
H_{0} \subset \mathfrak{n}_{0} \cap \mathfrak{z}(H)
$$

( $\mathfrak{z}$ is the center). The root space decomposition of $H$ is of course implicit in (1.2.5).

One should observe that quite generally, if we are given

$$
H_{ \pm j}, \quad j=0, \ldots, p
$$

arbitrary vector spaces such that $H_{j} \neq 0, j \neq 0$ and

$$
\beta_{j}: H_{j} \times H_{-j} \longrightarrow H_{0}, \quad j=1, \ldots, p,
$$

arbitrary bilinear mappings, we can construct a unique Lie algebra on the direct sum by the conditions

$$
H=\sum_{j=-p}^{p} H_{j}, \quad[x, y]=-[y, x]=\beta_{j}(x, y) \in H_{0}
$$


when $x \in H_{j}, y \in H_{-j}, j=1,2, \ldots, p$, and demand that all the other brackets are 0 . We shall call such an algebra an algebra of Heizenberg type. The algebra (1.2.7) is abelian if $\beta_{j}=0, j=1,2, \ldots, p$. The integer $p \geq 1$, which may not be uniquely determined, will be called the order of $H$.

The following facts are easy to verify:

Let $\{0\} \neq \tilde{H}_{j} \subset H_{j}, j= \pm 1, \ldots, \pm p, \tilde{H}_{0}=H_{0}$ be as abovr, then $\tilde{H}=\sum_{j=-p}^{p} \tilde{H}_{j} \triangleleft H$ is an ideal. If

$$
\sum_{j=1}^{p}\left[\tilde{H}_{j}, \tilde{H}_{-j}\right] \subset H_{0}^{*} \subset H_{0}
$$

where $H_{0}^{*}$ is an arbitrary subspace, then

$$
H^{*}=\sum_{\substack{j \neq 0 \\ j=-p}}^{p} \tilde{H}_{j}+H_{0}^{*}
$$

is an ideal of $\tilde{H}$ because of (1.2.6) (but not necessarily an ideal of $H$ ). If the spaces $H_{j}, H_{0}, \ldots$ are $\mathfrak{g}$ spaces as in (1.2.4), (1.2.5), then the above algebras are of course $\mathfrak{g}$-root algebras. In the above definition (1.2.8) the algebra $H^{*}$ could be the sum of an abelian algebra with an algebra of Heizenberg type of possibly lower order. It follows in particular that in the Heizenberg algebra (1.2.8) we can either find an abelian Heizenberg subalgebra of order 1

$$
H_{\alpha} \oplus H_{-\alpha}, \quad H_{ \pm \alpha} \neq 0, \quad\left[H_{\alpha}, H_{-\alpha}\right]=0,
$$

or a "purely non-abelian" subalgebra of Heizenberg type of order 1

$$
H_{\alpha} \oplus H_{-\alpha} \oplus H_{0}, \quad H_{ \pm \alpha} \neq 0, \quad H_{0}=\left[H_{\alpha}, H_{-\alpha}\right] \neq 0 .
$$

Furthermore, if $H$ is as in (1.2.4), (1.2.5) then we have $H_{ \pm \alpha} \subset \mathfrak{n}_{ \pm \alpha}$.

viii) Eigenvectors of a soluble action. Let $\mathfrak{g}$ be some soluble real Lie algebra that acts on the real vector space $V$ and therefore also on the complexified space $V_{c}=V \otimes \mathbb{C}$ (I use the notation ad for that action). By Lie's theorem we can then find

$$
0 \neq \xi=\zeta+i \eta \in V_{c}, \quad \operatorname{ad}(x) \xi=\lambda(x) \xi, \quad x \in \mathfrak{g},
$$


where $\zeta, \eta \in V$ and $\lambda \in \operatorname{Hom}_{\mathbb{R}}[\mathfrak{g}, \mathbb{C}]$. If $\lambda(x) \in \mathbb{R}$ then both $\zeta, \eta$ are common eigenvectors of the $\mathfrak{g}$ action, as long as they do not vanish. In general $\operatorname{Vec}_{\mathbb{R}}(\zeta, \eta) \subset V$ is a one or two dimensional $\mathfrak{g}$ subspace and the action of $\mathfrak{g}$ on $\operatorname{Vec}_{\mathbb{R}}(\zeta, \eta)$ is semisimple. Furthermore the action of $\operatorname{Exp}(\mathfrak{g}) \subset G L(V)$ is given, in real terms, by the composition of a dilation and a rotation (provided that the basis, and the the corresponding Euclidean structure, on that, one or two dimensional, subspace has been properly chosen). The above two operations of course commute with each other.

The final conclusion is that in both cases we can find in $V$ a one or two dimensional $\mathfrak{g}$-subspace on which the $\mathfrak{g}$ action is as above. We shall call such a subspace an eigenvalue subspace.

ix) The eigenvalue subalgebras. I shall specialize now the set up vii) in the case where $\mathfrak{n} \subset \mathfrak{q}$ and $\mathfrak{g}=\mathfrak{n}_{0}+\mathfrak{h}$ are as in vi) and I shall apply the considerations of viii) to the $\mathfrak{g}$-action on the $\mathfrak{g}$ subspaces of $\mathfrak{n}$.

It follows in particular that in each non zero subspace $\mathfrak{a}_{i}=\mathfrak{n}_{i} \cap \mathfrak{a}$, $i=0, \ldots, k$ of (1.2.2) we can find a one or two dimensional eigenvalue subspace $\tilde{\mathfrak{a}}_{i} \subset \mathfrak{a}_{i}$. We shall call the corresponding abelian algebra

$$
\tilde{\mathfrak{a}}=\sum \tilde{\mathfrak{a}}_{i} \subset \mathfrak{a}
$$

an eigenvalue abelian algebra.

Similarly if $0 \neq H_{ \pm \alpha} \subset \mathfrak{n}_{ \pm \alpha}$, as in (1.2.9), (1.2.10) we can find $\tilde{H}_{ \pm \alpha} \subset H_{ \pm \alpha}$ two eingenvalue subspaces with $\operatorname{dim} \tilde{H}_{ \pm \alpha}=1,2$. We can then consider

$$
\tilde{H}_{\alpha} \oplus \tilde{H}_{-\alpha} \oplus\left[\tilde{H}_{\alpha}, \tilde{H}_{-\alpha}\right]
$$

where now $0 \leq \operatorname{dim}\left[\tilde{H}_{\alpha}, \tilde{H}_{-\alpha}\right] \leq 4$. We shall call that algebra an eigenvalue algebra of Heizenberg type. This algebra could, of course, be abelian. The dimension of the algebra in (1.2.13) could be anything between 2 and 8 .

Observe finally that the action of $\mathfrak{g}$ on $W=\left[\tilde{H}_{\alpha}, \tilde{H}_{-\alpha}\right]$ is semisimple. Indeed the complexified space $W \otimes \mathbb{C}$ is generated over $\mathbb{C}$ by common eigenvectors of $\mathfrak{g}$. The eigenvalues of these vectors with respect to the action of $G=\operatorname{Exp}(\mathfrak{g}) \subset G L(W)$ are all unimodular, therefore the action of $G$ on $W$ is bounded and thus semisimple. In fact $W$ admits a direct $\mathfrak{g}$-decomposition $W=W_{1} \oplus \cdots$ such on each $W_{1}, \ldots$ the $G$ action is an orthogonal transformation. 
In both the above cases, the action of $\mathfrak{g}$ on the corresponding eigenvalue algebra is semisimple and abelian (i.e. it factors through $\mathfrak{g} \longmapsto \mathfrak{g} /[\mathfrak{g}, \mathfrak{g}]$ ). Furthermore the action of $\mathfrak{g} \cap \mathfrak{n}$ (which incidentally is equal to $\mathfrak{n}_{0}$ ) on the above eigenvalue algebra is both semisimple and nilpotent, it is therefore trivial.

\subsection{The Heart of the Matter: The algebraic reduction.}

The set up here will be the set up of a general $(\mathfrak{n}, \mathfrak{g})$ abstract root algebra

$$
\mathfrak{n}=\bigoplus_{e \in E} \mathfrak{n}_{e}, \quad e \in E
$$

\subsubsection{Bracket reduced algebras.}

We shall decompose the set of roots $E=A \cup B$ by

$A=\left\{e \in E: \mathfrak{n}_{e} \cap[\mathfrak{n}, \mathfrak{n}]=\{0\}\right\}, \quad B=\left\{e \in E: \mathfrak{n}_{e} \cap[\mathfrak{n}, \mathfrak{n}] \neq\{0\}\right\}$, and adopt throughout the notation

$$
\mathfrak{n}=\mathfrak{n}_{A}+\mathfrak{n}_{B}=\bigoplus_{\alpha \in A} \mathfrak{n}_{\alpha} \oplus \bigoplus_{\beta \in B} \mathfrak{n}_{\beta}
$$

It is then clear that $B=\varnothing$ if and only if $\mathfrak{n}$ is abelian. If $0 \in E$ we have

$$
\begin{aligned}
{\left[\mathfrak{n}_{\alpha}, \mathfrak{n}_{0}\right] \subset \mathfrak{n}_{\alpha} \cap[\mathfrak{n}, \mathfrak{n}] } & =\{0\}, \quad \alpha \in A \\
{\left[\mathfrak{n}_{A}, \mathfrak{n}_{0}\right] } & =\{0\}
\end{aligned}
$$

We shall say that $\mathfrak{n}$ is a bracket reduced algebra if

$$
[\mathfrak{n}, \mathfrak{n}]=\mathfrak{n}_{B}
$$

(Alternatively: for all $e \in E,[\mathfrak{n}, \mathfrak{n}] \cap \mathfrak{n}_{e}$ is either zero or $\mathfrak{n}_{e}$ ). Let $\mathfrak{n}$ be a bracket reduced algebra then

$$
\mathfrak{n}_{A}(\bmod [\mathfrak{n}, \mathfrak{n}])=\mathfrak{n}(\bmod [\mathfrak{n}, \mathfrak{n}])
$$


and this, by the nilpotency of $\mathfrak{n}$, implies that $\mathfrak{n}_{A}$ generates $\mathfrak{n}$. In particular

$$
\begin{aligned}
& {[\mathfrak{n}, \mathfrak{n}]=\mathfrak{n}_{B}=\sum\left[\mathfrak{n}_{A},\left[\mathfrak{n}_{A},\left[\ldots, \mathfrak{n}_{A}\right], \ldots\right],\right.} \\
& B \subset \sum_{j \geq 2}(A+A+\cdots+A),
\end{aligned}
$$

where $j$ under the summation indicates the length of the summation $A+\cdots+A$. By (1.3.2) and (1.3.3) it follows that if $0 \in E$ and $\mathfrak{n}$ is bracket reduced, then we have

$$
\mathfrak{n}_{0} \subset \mathfrak{z}(\mathfrak{n})
$$

We also have:

Proposition. A bracket reduced algebra for which $B=\{0\}$ is the direct sum of an abelian algebra and an algebra of Heizenberg type (cf. 1.2.vii)).

Indeed by (1.3.4) and the hypothesis it follows that

$$
[\mathfrak{n}, \mathfrak{n}] \subset \mathfrak{z}(\mathfrak{n})
$$

But this together with (1.3.3) and the hypothesis implies that $\mathfrak{n}=$ $[\mathfrak{n}, \mathfrak{n}]$ and that

$$
[\mathfrak{n}, \mathfrak{n}]=\sum\left\{\left[\mathfrak{n}_{\alpha}, \mathfrak{n}_{-\alpha}\right], \alpha,-\alpha \in A\right\}
$$

Clearly the proposition is but a reformulation of (1.3.5), (1.3.6).

\subsubsection{The bracket reduction.}

Let $\mathfrak{n}=\mathfrak{n}_{A}+\mathfrak{n}_{B}$ be an arbitrary root algebra as in 1.3.1, and let

$$
\mathfrak{n}_{1}=\mathfrak{n}_{A}+[\mathfrak{n}, \mathfrak{n}]
$$

which is an ideal in $\mathfrak{n}$ and also a $\mathfrak{g}$-root algebra with the same root set $E_{1}=E$, i.e. in the process of passing from $\mathfrak{n}$ to $\mathfrak{n}_{1}, \mathfrak{n} \rightarrow \mathfrak{n}_{1}$ we "have 
not lost any roots". We clearly have for $\mathfrak{n}_{1}$ the analog of the (1.3.1) decomposition

$$
\mathfrak{n}_{1}=\mathfrak{n}_{A_{1}} \oplus \mathfrak{n}_{B_{1}}
$$

and clearly

$$
A \subset A_{1}, \quad B \supset B_{1}, \quad E=A \cup B=A_{1} \cup B_{1}=E_{1} .
$$

Furthermore, $\mathfrak{n}=\mathfrak{n}_{1}$ if and only if $\mathfrak{n}$ is bracket reduced.

The operation $\mathfrak{n} \longmapsto \mathfrak{n}_{1}$ can clearly be iterated $\mathfrak{n} \longrightarrow \mathfrak{n}_{1} \longrightarrow$ $\left(\mathfrak{n}_{1}\right)_{1}=\mathfrak{n}_{2} \longrightarrow \cdots$ until we stop $\mathfrak{n} \triangleright \mathfrak{n}_{1} \triangleright \cdots \triangleright \mathfrak{n}_{p}=\mathfrak{n}_{p+1}=\mathfrak{n}^{*}$. We obtain thus $\mathfrak{n}^{*} \subset \mathfrak{n}$ a subnormal bracket reduced subalgebra

$$
\mathfrak{n} \triangleright \triangleright \mathfrak{n}^{*}=\mathfrak{n}_{A^{*}}+\mathfrak{n}_{B^{*}}, \quad A \subset A^{*}, \quad B \supset B^{*}, \quad A^{*} \cup B^{*}=E .
$$

\subsubsection{The $\mathcal{A}$-reduction.}

We shall now consider $\mathfrak{n}=\mathfrak{n}_{A}+\mathfrak{n}_{B}(E=A \cup B)$ some bracket reduced root algebra as in (1.3.1) that is assumed to be an $\mathcal{A}$-algebra, i.e. $E \in \mathcal{A}$ where $\mathcal{A}$ is as in Section 1.1.2.

We shall consider the couple of subsets

$$
A \subset E \subset V
$$

and we shall distinguish two mutually exclusive possibilities.

Case i) $A \subset E$ is a minimal $\mathcal{A}$-couple.

Case ii) There exists $0 \neq \alpha \in A$ such that $E \backslash\{\alpha\} \in \mathcal{A}$.

The fact that $E \subset A+A+\cdots$ (cf. (1.3.3) shows that $\mathfrak{n}$ falls either under case i) or case ii). Let us assume that $\mathfrak{n}$ is as in case ii) and that $E \backslash\{\alpha\} \in \mathcal{A}$. We can consider then

$$
\mathfrak{n}^{\alpha}=\mathfrak{n} \ominus \mathfrak{n}_{\alpha}=\mathfrak{n}_{A \backslash\{\alpha\}}+\mathfrak{n}_{B} \triangleleft \mathfrak{n},
$$

which is an ideal of $\mathfrak{n}$ and also an $\mathcal{A}$-algebra where the set of roots is $E \backslash\{\alpha\}$. In general $\mathfrak{n}^{\alpha}$ is not bracket reduced. We shall consider therefore the subalgebra

$$
\mathfrak{n}^{1}=\left(\mathfrak{n}^{\alpha}\right)^{*} \triangleleft \triangleleft \mathfrak{n}
$$


This is a bracket reduced $\mathcal{A}$-algebra.

If $\mathfrak{n}$ is as in case $\mathrm{i}$ ) we set $\mathfrak{n}^{1}=\mathfrak{n}$ so that $\mathfrak{n}=\mathfrak{n}^{1}$ if and only if we are in case i).

This operation $\mathfrak{n} \longmapsto \mathfrak{n}^{1} \longrightarrow\left(\mathfrak{n}^{1}\right)^{1}=\mathfrak{n}^{2} \longrightarrow \cdots$ can be iterated until it becomes stationary: $\mathfrak{n}^{p}=\mathfrak{n}^{p+1}=\hat{\mathfrak{n}}$. We have thus proved the following.

Proposition. Let $\mathfrak{n}$ be some $\mathcal{A}$ root algebra, we can then find

$$
\hat{\mathfrak{n}}=\hat{\mathfrak{n}}_{\hat{A}}+\hat{\mathfrak{n}}_{\hat{B}} \triangleleft \triangleleft \mathfrak{n},
$$

some subnormal bracket reduced $\mathcal{A}$-subalgebra, such that $\hat{A} \subset \hat{A} \cup \hat{B}=\hat{E}$ is a minimal $\mathcal{A}$-couple.

In the special case when $\mathcal{A}=\mathcal{A}_{1} \cap \mathcal{A}_{2}$ as in Section 1.1.4 we know that there are exactly two possibilities:

i) $\hat{B}=\varnothing$ : the subalgebra $\hat{\mathfrak{n}}$ is then abelian.

ii) $\hat{B}=\{0\}$ : the algebra $\hat{\mathfrak{n}}$ contains a subalgebra (possibly abelian) of Heizenberg type as in (1.2.9) or (1.2.10).

\subsubsection{The eigenvalue subalgebra.}

Let us specialize further and consider the case $\mathfrak{n} \subset \mathfrak{q}$ and $\mathfrak{g}=\mathfrak{n}_{0}+\mathfrak{h}$ as in 1.2.vi). If by the above reduction (i.e. as in the proposition of Section 1.3.3) we are in case 1.2.vii) ${ }_{a}$ we can proceed as in (1.2.12) and find a possibly smaller subnormal subalgebra that is an eigenvalue algebra and whose roots $\hat{\hat{A}} \subset \hat{A}$ are the vertices of a simplex in $(\mathfrak{h} / \mathfrak{h} \cap$ $\mathfrak{n})^{*}$ such that $0 \in \operatorname{Int}[\hat{\hat{A}}]$ (in particular none of the roots is 0 ).

If by the above reduction we are in case 1.2 .vii ${ }_{h}$ we can proceed as in (1.2.13) and find a possibly smaller eigenvalue subalgebra that is of Heizenberg type.

The above eigenvalue subalgebras are of course not uniquely determined. We shall fix $\mathfrak{e} \subset \mathfrak{n}$, once and for all, one such algebra and we shall denote by $r$ the number of its distinct roots (i.e. with our previous notations, $r=\operatorname{card}(\hat{\hat{A}})$ in the abelian case, and $r=2$ in the Heizenberg case). We shall also fix some basis $e_{1}, \ldots, e_{q}$ of $\mathfrak{e}$ where

$$
q \leq m=\operatorname{dim} \mathfrak{n} \leq n=\operatorname{dim} \mathfrak{q}
$$


That basis will be chosen and fixed once and for all so as to have the following additional properties:

i) If $\mathfrak{e}$ is abelian as in (1.2.12): For each $1 \leq i \leq r$ we can find $1 \leq j \leq q$ such that

$$
e_{j} \text { or }\left(e_{j}, e_{j+1}\right) \subset \tilde{\mathfrak{a}}_{i},
$$

depending on whether $\operatorname{dim} \tilde{\mathfrak{a}}_{i}=1$ or 2 , and $e_{j}$ or $\left(e_{j}, e_{j+1}\right)$ is a basis of that subspace.

ii) If $\mathfrak{e}$ is a Heizenberg algebra as in (1.2.13): For each of the two subspaces $H_{\alpha}, H_{-\alpha}$ we can find $1 \leq j \leq q$ such that

$$
e_{j} \text { or }\left(e_{j}, e_{j+1}\right) \subset H_{ \pm \alpha},
$$

and such that $e_{j}$ or $\left(e_{j}, e_{j+1}\right)$ is a basis of that subspace. This will dispose of at most $e_{1}, e_{2}, e_{3}, e_{4}$ and at last $e_{1}, e_{2}$. The remaining elements of the basis lie in $\left[\tilde{H}_{-\alpha}, \tilde{H}_{\alpha}\right]$ and form a basis of that space.

When

$$
\operatorname{dim} \tilde{\mathfrak{a}}_{i}, \quad \operatorname{dim} H_{ \pm \alpha}=2,
$$

in (1.3.7) or (1.3.8), we shall further impose on the pair $e_{j}, e_{j+1}$ the condition that with respect to that basis the action of Exp $\mathfrak{g}$ on $\tilde{\mathfrak{a}}_{i}$ or $H_{ \pm \alpha}$ is a composition of a dilation and a euclidean rotation as in 1.2.viii). It is also possible to choose that basis so that the action of Exp $\mathfrak{g}$ on $\left[\tilde{H}_{-\alpha}, \tilde{H}_{\alpha}\right]$ can be split into a number of rotations. This last point is however not vital for what follows.

The above notations of $\mathfrak{e}$ of $r, q, m, n$ and of the above basis will be fixed for the rest of the paper.

\section{Lie Group Considerations.}

\subsection{The Exponential basis.}

Let $\mathfrak{q}$ be some soluble real Lie algebra and let $e_{1}, \ldots, e_{n} \in \mathfrak{q}$ be a basis of $\mathfrak{q}$ such that the subspaces $I_{j}=\operatorname{Vec}\left[e_{1}, \ldots, e_{j}\right]$ satisfy the condition

$$
\left[I_{j+1}, I_{j}\right] \subset I_{j}, \quad j=1,2, \ldots
$$

We shall call such a basis an exponential bases of $\mathfrak{q}$. 
Let now $\mathfrak{e} \subset \mathfrak{n}, \mathfrak{h} \subset \mathfrak{q}$ be as in 1.2.vi) and 1.3.4. We shall consider in what follows special exponential basis and denote them

$$
\left\langle e_{1}, \ldots, e_{m}, u_{1}, \ldots, u_{s}\right\rangle \subset \mathfrak{q},
$$

where with the notations of 1.3 .4 we have:

i) $e_{1}, \ldots, e_{q}$ is a basis of $\mathfrak{e}$ as in 1.3.4.

ii) $e_{1}, \ldots, e_{m}$ is an exponential basis of $\mathfrak{n}$ and $m \geq q$.

iii) $u_{1}, \ldots, u_{s} \in \mathfrak{h}$ and $m+s=n$.

To guarantee iii), recall that $\mathfrak{n}+\mathfrak{h}=\mathfrak{q}$ and that $[\mathfrak{h}, \mathfrak{h}] \subset \mathfrak{n}$ and therefore any set $u_{1}, \ldots, u_{s} \in \mathfrak{h}, s=n-m$, such that $e_{1}, \ldots, e_{m}$, $u_{1}, \ldots, u_{s}$ is a basis of $\mathfrak{q}$ will give an exponential basis.

A special choice of $u_{1}, \cdots, u_{s}$ will be made in what follows. Towards that let us consider the space $V=\mathfrak{h} / \mathfrak{h} \cap \mathfrak{n}$ and identify $V=V^{*}$, once and for all, by some fixed scalar product. We shall choose appropriately $u_{1}, \ldots, u_{s}$ some basis of $V$ and then lift it in anyway whatsoever so as to form the basis (2.1.1). To do that, we consider $L_{1}, \cdots, L_{k} \in V^{*}$ the distinct non-zero real roots of the action of $\mathfrak{h}$ on $\mathfrak{n}$ and consider the subset $L_{1}, \ldots, L_{r}$ of these roots that was constructed in 1.3.4 and which gives the distinct roots of the eigenvalue algebra $\mathfrak{e}$.

If $\mathfrak{e}$ is abelian as in (1.2.12) we can assume that $L_{1}, \ldots, L_{r}$ are the vertices of a simplex (equal to $[\hat{\hat{A}}]$ with the notations of 1.3.4) and

$$
0 \in \operatorname{Int}\left[L_{1}, \ldots, L_{r}\right] .
$$

If $\mathfrak{e}$ is of Heizenberg type as in (1.2.13) we can assume that $r=2$, $L_{1}=-L_{2} \neq 0$.

With the identification of $V=V^{*}$ we shall identify $L_{1}, \ldots, L_{r}$ with elements of $V$ and we shall set

$$
\begin{gathered}
V_{2}=\operatorname{Vec}\left[L_{1}, \ldots, L_{r}\right], V_{1}=V_{2}^{\perp}=\left[u \in V, L_{j}(u)=0,1 \leq j \leq r\right], \\
V=V_{1} \oplus V_{2}, \quad 0 \leq \operatorname{dim} V_{1}=\sigma \leq s, \\
s-\sigma=\operatorname{dim} V_{2}=r-1 \text { if } \mathfrak{e} \text { is abelian as in (1.2.12), } \\
s-\sigma=\operatorname{dim} V_{2}=1 \text { if } \mathfrak{e} \text { is of Heizenberg type as in (1.2.13). }
\end{gathered}
$$

In both cases we choose $u_{1}, \ldots, u_{\sigma}$ to be a basis of $V_{1}$ and $u_{\sigma+1} \cdots u_{s}$ to be a basis of $V_{2}=V_{1}^{\perp}$. The notations $\sigma, s$ for these two dimensions will be mentioned throughout. 
A special case: Split algebras. We say that $\mathfrak{q}$ is a split algebra if it is possible to choose $\mathfrak{h}$ as above to be an abelian algebra. This is for instance the case when $\mathfrak{q}$ is the Lie algebra of a real algebraic group. In that case, the basis elements $u_{1}, \cdots, u_{s}$ chosen above, commute and span an abelian subalgebra $V \subset \mathfrak{q}$ that gives a semidirect product decomposition:

$$
\mathfrak{q}=\mathfrak{n} \bowtie V
$$

REMARK. The notation for semidirect product in [2] is $\lambda$ and in [1] it is $X_{\sigma}$.

The use of the above extraneous scalar products on $V$ can be avoided. $c f$. Section 3.2.3 for $C$-graphs.

\subsection{The Exponential Coordinates.}

If $e_{1}, e_{2}, \ldots, e_{n} \in \mathfrak{q}$ are exponential coordinates of $\mathfrak{q}$ as in Section 2.1 we can use them to identify $Q$, the simply connected soluble group that corresponds to $\mathfrak{q}$, with $\mathbb{R}^{n}$ by the identification $(c f .[1]$ )

$$
\mathbb{R}^{n} \ni\left(t_{1}, \ldots, t_{n}\right) \longrightarrow \operatorname{Exp}\left(t_{1} e_{1}\right) \cdots \operatorname{Exp}\left(t_{n} e_{n}\right) \in Q
$$

If we use the special exponential basis constructed in Section 2.1 we obtain a number of important identifications. Let $N, H, N_{E} \subset Q$ be the subgroups that correspond to $\mathfrak{n}, \mathfrak{h}, \mathfrak{e} \subset \mathfrak{q}$. Let $V, V_{1}, V_{2} \subset Q$ be the submanifolds that correspond to $V, V_{1}, V_{2}$ identified to subspaces of $\mathbb{R}^{n}$ by the identification (2.2.1). We have

$$
V_{1}, V_{2} \subset V \subset H, \quad N \cdot V=N \cdot V_{1} \cdot V_{2}=Q,
$$

where "." indicates group multiplication. When $\mathfrak{q}$ is a split algebra we have a semidirect product decomposition

$$
\begin{gathered}
Q=N \bowtie V=\left(N \bowtie V_{1}\right) \bowtie V_{2}, \\
Q \supset N_{E} \bowtie V=\left(N_{E} \bowtie V_{1}\right) \bowtie V_{2}=Q_{E},
\end{gathered}
$$

where in this special case $V_{1}, V_{2} \subset V \subset Q$ are subgroups ( $\cong$ to vector spaces).

In the general case we have $C^{\infty}$-manifold identifications

$$
Q \cong N \times V \cong N \times V_{1} \times V_{2} \supseteq N_{E} \times V_{1} \times V_{2}=Q_{E}
$$


but of course in general $Q_{E}$ cannot be identified to a subgroup of $Q$.

Observe however, that in the case of the Heizenberg eigenvalue algebra (1.2.13), $\operatorname{dim} V_{2}=1$ and then $V_{2} \subset Q$ is a subgroup and so is $N_{E} \bowtie V_{2}$.

Observe finally that the fact that $\mathfrak{h} \cap \mathfrak{n} \subset \mathfrak{n}_{0}$ in 2.2.v) implies that if we are in the abelian case (1.2.12) we have

$$
N_{E} \cap H=\{e\} .
$$

If we denote by $G_{p}(V) \subset H$ the subgroup generated by $\mathrm{V}$, this implies that we have

$$
N_{E} \cap G p(V)=\{e\}
$$

and that these two groups form a semidirect product in $Q$

$$
N_{E} \bowtie G p(V) \subset Q
$$

Observe also that by the final remark of 1.2.ix), whether $N_{E}$ is abelian or not, the action of $G p(V)$ on $N_{E}$ factors through $Q \longrightarrow Q / N$. Also, by the definition of $V_{1}, \operatorname{Ad}_{\mathfrak{e}}\left(v_{1}\right), v_{1} \in V_{1}$, lie in some compact subgroup of $G L(\mathfrak{e})$.

\subsection{Riemannian structures on Lie groups.}

On every connected Lie group we can assign a unique, up to quasiisometry, left invariant Riemannian structure by assigning some fixed scalar product on the Lie algebra of $G$. I shall denote by $d(\cdot, \cdot)=d_{G}(\cdot, \cdot)$ the corresponding distance and by $|x|=d(x, e)$.

It is of course clear that if $\varphi: G_{1} \longrightarrow G_{2}$ is a group homomorphism then $d \varphi$ is bounded and if $\varphi$ identifies $G_{1}$ to a closed subgroup of $G_{2}$ then $d \varphi$ is quasiisometric. When $G=G_{1} \bowtie G_{2}$ is a semidirect product $\left(G \ni g=g_{1} g_{2}\right)$, by identifying $G=G_{1} \times G_{2}$, we clearly have $T G \cong$ $T G_{1} \times T G_{2}$ canonically. That identification induces an isometry on $T G_{2}$ (but in general not on $T G_{1}$ ) and $T G_{1} \perp T G_{2}$ in $T G$. The above hold, of course, for an appropriate choice of the corresponding left invariant Riemannian structures.

If $G$ is soluble and simply connected, we can identify it to $\mathbb{R}^{n}$ as in (2.2.1) and assign on $G$ the corresponding Euclidean Riemannian structure and the corresponding distance $d_{e}(\cdot, \cdot)$. It is an immediate 
consequence of the Baker-Campbell-Hausdorff formula [1] that we have

$$
\begin{gathered}
A^{-1} d_{G}(x, y) \leq d_{e}(x, y) \leq A d_{G}(x, y), \\
A=C+|x|^{C}+|y|^{C}, \quad x, y \in G,
\end{gathered}
$$

provided that $G$ is nilpotent and where $C>0$ is independent of $x, y$.

If we identify $V \subset G$ with $\mathbb{R}^{s}$ as in (2.2.2) we deduce from the fact that $V \subset H$ and the fact that $G / N \cong V \cong \mathbb{R}^{s}$ (this implies that for $x \in$ $V,|x|$ is equivalent to the Euclidean norm) that the analogous estimate (2.3.1), (2.3.2) holds for $x, y \in V$, and that we can even take $A=C$ independent of $x, y$ in the split case $G=N \bowtie V$. A consequence of the above is that the Euclidean Riemannian structure and the Riemannian structure induced on $V, V_{1}, V_{2}$ by the identifications (2.2.3)

$$
V \longrightarrow n \times V \subset G, \quad n \in N
$$

are "polynomially distorted" (uniformly in $n \in N$, i.e. the ratio of the two Riemannian norms on the tangent space at $x \in V$ can be bounded by $C|x|^{C}+C$ ) in general, and quasiisometric in the split case.

Let the notations be as in $(2.2 .2),(2.2 .3)$. When $V_{2} \subset Q$ is a subgroup, we can give on $N_{E} \times V_{2} \cong N_{E} \bowtie V_{2}$ two Riemannian scalar products $\langle\cdot, \cdot\rangle_{\bowtie}$ and $\langle\cdot, \cdot\rangle_{u_{1}}, u_{1} \in V_{1}$ on the tangent space. $\langle\cdot, \cdot\rangle_{\bowtie}$ is the left invariant structure of the group $N_{E} \bowtie V_{2}$. The definition of $\langle\cdot, \cdot\rangle_{u_{1}}$ does not depend on the fact that $V_{2} \subset Q$ is a subgroup and is the Riemannian structure induced by the embedding

$$
N_{E} \times V_{2} \ni\left(n, u_{2}\right) \underset{I_{u_{1}}}{\longrightarrow}\left(n, u_{1}, u_{2}\right) \in N_{E} \times V_{1} \times V_{2} \subset Q
$$

and by the left invariant Riemannian structure of $Q$. Even when $V_{2}$ is not a subgroup we can still define $\langle\cdot, \cdot\rangle_{\bowtie}$ on $N_{E} \times V_{2}$ as follows. The embedding of $\mathbb{R}^{s} \cong V=Q / N \subset Q$ defined in (2.2.2) induces, by the final remark of Section 2.2, an action of $Q / N$ on $N_{E}$. That action can be used to define a group $N_{E} \bowtie(Q / N)$. That group can, in turn, be used to define a left invariant Riemannian structure and therefore the corresponding $\langle\cdot, \cdot\rangle_{\bowtie}$ on $T\left(N_{E} \times V\right)$ and $T\left(N_{E} \times V_{2}\right)$. Although it is not essential for what follows, one can observe at this point that the group structure $N_{E} \bowtie(Q / N)$ does not depend on the particular embedding (2.2.2) and that the above Riemannian structure on $N_{E} \times V$ is intrinsically defined. To see this it suffices to use the final remark of 
Section 1.2 (ix) and the fact that $\mathfrak{h} \cap \mathfrak{n}$ acts on $\mathfrak{e}$ nilpotently. These two facts put together show that the $\mathfrak{h} \cap \mathfrak{n}$ acts trivially on $\mathfrak{e}$.

We shall denote by $|\cdot|_{\bowtie}$ and $|\cdot|_{u_{1}}$ the corresponding norms on $T\left(N_{E} \times V_{2}\right)$. We have then

Lemma 2.3.1. At every point $\left(n, u_{2}\right) \in N_{E} \times V_{2}$ and for every $u_{1} \in V_{1}$, we have

$$
A^{-1}|\cdot|_{u_{1}} \leq|\cdot|_{\bowtie} \leq A|\cdot|_{u_{1}},
$$

where

$$
A=C\left|u_{2}\right|^{C}+C,
$$

where $C>0$ is independent of $n, u_{1}, u_{2}$. Furthermore, in the split case $Q=N \bowtie V$ we can take $A=C$.

Proof. With the notations (2.3.3) and the identifications (2.2.3) we have

$$
I_{u_{1}}\left(n, u_{2}\right)=n \cdot u_{1} \cdot u_{2}=u_{1} \cdot n^{u_{1}} \cdot u_{2} \in Q ，
$$

where "." denotes the group product and $n^{u_{1}}=u_{1}^{-1} n u_{1}$ denotes the inner action of $u_{1}$ on $N_{E}$. It follows that

$I_{u_{1}^{\prime}}=$ Left multiplication by $u_{1}^{\prime} u_{1}^{-1} \circ I_{u_{1}} \circ\left[\left(n \longmapsto n^{u_{1}^{\prime} u_{1}^{-1}}\right) \times\right.$ Identity $]$.

We conclude therefore from the left invariance of the Riemannian structures on $Q$, that it suffices to prove (2.3.4) with $u_{1}=0$ (cf. final remark of Section 2.2).

When $u_{1}=0, I_{0}$ identifies $N_{E} \times V_{2}$ to a submanifold of $N_{E} \bowtie$ $G p\left(V_{2}\right)(c f .(2.2 .4))$. This means that for both Riemannian structures $\langle\cdot, \cdot\rangle_{\bowtie}$ and $\langle\cdot, \cdot\rangle_{0}$, with the canonical identifications, we have

$$
T N_{E} \perp T V_{2}
$$

By the definition of the action of $V_{2}$ on $N_{E}$ in the group $N_{E} \bowtie V_{2}$ which is identical to the action of $V_{2} \subset Q$ on $N_{E}$ we see that

$$
|\xi|_{\bowtie}=|\xi|_{0}, \quad \xi \in T N_{E}
$$

The polynomial distortion in (2.3.5) in the Lemma is therefore a consequence of $(2.3 .1),(2.3 .2)$ and the few lines that follow. 
Let us denote by

$$
\begin{aligned}
\Phi: X= & \left(N_{E} \times V_{2}\right) \times V_{1} \longrightarrow Q \\
& {\left[\left(n, u_{2}\right), u_{1}\right] \longmapsto n \cdot u_{1} \cdot u_{2} \quad \text { (group product) } . }
\end{aligned}
$$

Let us assign $N_{E} \times V_{2}$ with the $\langle\cdot, \cdot\rangle_{\bowtie}$ Riemannian structure, $V_{1}$ with the Euclidean Riemannian structure, $X$ with the product structure and $Q$ with the left invariant Riemannian structure. With these notations we have proved the first part (i.e. (2.3.6)), of the following

Lemma 2.3.2. The differential of $\Phi$ satisfies at $x=\left[\left(n, u_{2}\right), u_{1}\right] \in X$

$$
\begin{gathered}
\left\|\left.d \Phi\right|_{T\left(N_{E} \times V_{2}\right)}\right\| \leq C\left(\left|u_{2}\right|^{c}+C\right), \\
\left\|\left.d \Phi\right|_{T V_{1}}\right\| \leq C\left(\left|u_{1}\right|^{c}+\left|u_{2}\right|^{c}+C\right),
\end{gathered}
$$

where $C>0, c \geq 0$ are independent of $x$. Furthermore, when $Q=N \bowtie$ $V$ is a split group, we can take $c=0$.

The assertion (2.3.7) is once more a consequence of the few lines that follow (2.3.1), (2.3.2).

\subsection{A special class of groups and explicit coordinates.}

In this section we shall consider two important classes of groups

i) $G=\mathbb{R}^{r} \bowtie V=\mathbb{R}^{r} \bowtie \mathbb{R}^{s}$ where the action of $V$ on $\mathbb{R}^{r}$ is given by

$$
\operatorname{Ad}_{\mathbb{R}^{r}}(y)=\left(\begin{array}{ccc}
\exp \left(L_{1}(y)\right) & & 0 \\
& \ddots & \\
0 & & \exp \left(L_{r}(y)\right)
\end{array}\right), \quad y \in V,
$$

where $L_{1}, \ldots, L_{r} \in V^{*}$. This group, after the identification with $\mathbb{R}^{r+s}$ with the obvious exponential coordinates, gives a Riemannian structure on $\mathbb{R}^{r+s}$ with an orthonormal basis at $\left(x_{1}, \ldots, x_{r}, y_{1} \ldots, y_{s}\right)$

$$
\left(\exp \left(L_{1}(y)\right) \frac{\partial}{\partial x_{1}}, \ldots, \exp \left(L_{r}(y)\right) \frac{\partial}{\partial x_{r}} ; \frac{\partial}{\partial y_{1}}, \ldots, \frac{\partial}{\partial y_{s}}\right) \subset T G
$$


The left invariant Riemannian structure $\langle\cdot, \cdot\rangle_{\bowtie}$ induced on $N_{E} \bowtie V_{2}$, as we have considered in the previous section, is clearly of this kind when $N_{E}$ is abelian (as in (1.2.12)). The imaginary part of the roots play no role as far as the Riemannian structure is concerned (they just give rise to orthogonal rotations in the 2-dimensional root spaces if there are any).

ii) It is a little less simple to write down the orthonormal basis of $G=N_{E} \bowtie V_{2} \subset Q$ when $N_{E}$ is an eignevalue algebra of Heizenberg type as in (1.2.13). We shall not need to do this, we shall only need a simple geometric estimate. In fact, here we might as well consider a group of the form $N \bowtie V=N \bowtie \mathbb{R}$ where $N$ is an arbitrary simply connected nilpotent group, and not just a group of Heizenberg type, and $\operatorname{dim} V=1$ (such groups are called of "rank 1"). We shall then fix $e \in \mathfrak{n}$ (the Lie algebra of $N$ ) and assume that

$$
|\operatorname{Ad}(y) e|_{\mathfrak{n}} \leq \exp (-\alpha y), \quad y \geq 0
$$

for some $\alpha>0$, where $\mathbb{R}$ has been identified with $V$. This will certainly be the case for $N_{E} \bowtie V_{2}$ in Section 2.2 and $e$ the basis vectors in $H_{ \pm \alpha}$ of (1.3.8) (provided that in the identification of $V_{2}$ with $\mathbb{R}$ we have chosen the right orientation. These orientations are, of course, opposite for $H_{\alpha}$ and $H_{-\alpha}$ ).

Let us now consider the "path"

$$
\varphi: \mathbb{R} \ni \tau \longrightarrow(n \operatorname{Exp}(\tau e), y) \in N \bowtie V=G,
$$

for fixed $n \in N, y>0$. We then clearly have

$$
\varphi(\tau)=(n, y) \operatorname{Exp}(\tau \operatorname{Ad}(y) e)
$$

and therefore

$$
|\dot{\varphi}(\tau)|=\left|d \varphi\left(\frac{\partial}{\partial \tau}\right)\right| \leq \exp (-\alpha y)
$$

for the Riemannian norm $|\cdot|$ on $T G$. 


\section{Geometric Considerations.}

\subsection{Notations and Definitions.}

I found it very difficult to describe the Geometric and Topological constructions that are presented in this part of the paper without having to resort to informal language, and without constantly abusing the notations that I had already established. The aim of this first section is to codify as far as possible, some of the notations and the notions that will be needed and used in the rest of this paper.

I shall use the notation

$$
\square_{d}^{r}=\left[\left(x_{1}, \ldots, x_{r}\right) \in \mathbb{R}^{r},\left|x_{j}\right| \leq d, 1 \leq j \leq r\right] \subset \mathbb{R}^{r}
$$

with $r=1,2, \ldots$, for the $d$-cube. This $d \geq 10^{10}$ will be the free parameter in this paper and none of the constants $C>0$ that will appear will depend on $d$. I will also denote by $\square_{1}^{r}$ the above cube for $d=10^{10}$. (Which is the "unit" cube for you if you happen to be $1.8 \times 10^{10}$ meters tall.) Together with the above cube I shall also consider anisotropic cubes of the form

$$
\square_{1}^{r} \times[-A, A]^{t},
$$

where $A \leq C(\log d)^{C}$. I shall denote by $\partial \square_{d}^{r}$ for the topological boundary of (3.0.1) in $\mathbb{R}^{r}$, with an analogous definition for (3.0.2).

Let now $M$ be some Riemannian manifold and let

$$
\Phi: \square_{d}^{r} \longrightarrow M
$$

be some $\operatorname{Lip}(C \ell)$ mapping defined in some neighbourhood of $\square_{d}^{r}$ in $\mathbb{R}^{r}$, where here and throughout, the subsets of $\mathbb{R}^{r}$ are assigned with the Euclidean distance. One should think here of $\ell>1$ as a free parameter that may be allowed to $\longrightarrow \infty$. When we have (3.0.3) we shall say that $E$, the image of $\square_{d}^{r}$ by $\Phi$, is

$$
E=\Phi\left(\square_{d}^{r}\right) \text { is a } \operatorname{Lip}(\ell)-\square_{d}^{r} \subset M
$$

The notation (3.0.4) to describe (3.0.3) is already abusive but convenient. We shall use an analogous definition for

$$
\Phi(X) \text { is a } \operatorname{Lip}(\ell)-X \subset M
$$


where $X$ is as (3.0.2) or $X=\partial \square_{d}^{r}$ or $X=\partial\left(\square_{d}^{r} \times[-A, A]^{t}\right)$, etc.

In this context we shall use the following obvious scaling property: If

$$
E \text { is a } \operatorname{Lip}(\ell)-\square_{1}^{r} \times[-A, A]^{t} \subset M,
$$

then automatically

$$
E \text { is a } \operatorname{Lip}(\ell(A+1))-\square_{1}^{r+t} \subset M \text {. }
$$

The analogous property for any $X$ as in (3.0.5) holds.

I shall use throughout the notation $L L(d)$ to indicate mappings from one metric space to another that are $\operatorname{Lip}\left(C(\log d)^{C}\right)$ for some $C>0$.

One of the basic definitions given in the introduction (Section 0) will be reformulated as follows. We shall say that the Riemannian manifold $M$ has property $\mathcal{F}_{r}, r \geq 2$ (we shall also denote $\mathcal{F}=\bigcap_{p \geq 2} \mathcal{F}_{p}$ ) if for every

$$
E=\Phi\left(\partial \square_{1}^{r}\right) \text { is a } L L(d)-\partial \square_{1}^{r} \subset M
$$

we can find some $\hat{E}$

$$
\hat{E}=\hat{\Phi}\left(\square_{1}^{r}\right) \text { is a } L L(d)-\square_{1}^{r} \subset M
$$

that "fills in" $E$. The meaning of "fills in" is the following

$$
\left.\hat{\Phi}\right|_{\partial \square_{1}^{r}}=\Phi,
$$

we have in particular $E \subset \hat{E}$.

I shall not make systematic use of the notations from the Theory of currents ( $c f .[12],[13])$, because it is not necessary for our constructions or for the proof of our theorem to introduce an orientation in $\mathbb{R}^{r}$. But if we do orient $\mathbb{R}^{r}$ then (3.0.8) and (3.0.9) define currents $E$ and $\hat{E}$ in $M$, and (F.I.) says among other things that

$$
\partial \hat{E}=E
$$

for the $\partial$-operator ( $c f .[13]$; sometimes denoted by $b$-operator $c f . ~[12]$ ) of the currents, provided of course that the orientations of $E$ and $\hat{E}$ are compatible. 


\subsection{The One Dimensional Construction.}

\subsubsection{The abelian case.}

This construction will be made in the group $G=\mathbb{R}^{2} \bowtie \mathbb{R}$ of 2.4.i) (with $r=2, s=1$ ) where $L_{1}=\alpha L_{2} \neq 0,(\alpha \in \mathbb{R})$.

The issue is to give a specific embedding of $\partial \square_{d}^{2}$ in $G$ that will have a number of properties.

1) The four vertices $\left.( \pm d, \pm d) \in \partial \square_{d}^{2}\right)$ will be mapped on the corresponding points $( \pm d, \pm d, 0) \in G$, where the exponential coordinates $\left(x_{1}, x_{2}, y\right)$ of 2.4.i) are used throughout for the group $G$.

2) The four sides $f_{i}, 1 \leq i \leq 4$ (i.e. 1-dimensional faces of $\square_{d}^{2}$ ) will be mapped into four $C^{\infty}$ curves $\gamma_{i} \subset G,(1 \leq i \leq 4)$ that join the corresponding vertices. Say the side $f_{1}$ of $\square_{d}^{2}$ that joins $(d, d)$ to $(-d, d)$, is mapped on $\gamma_{1} \subset G$ that joins $(d, d, 0),(-d, d, 0)$. The curve $\gamma_{i}$ lies in the affine hyperplane $A_{i}$ that is parallel to the $y$-axis and which goes through $f_{i} \subset \mathbb{R}^{2} \subset G$ (with the above identification). The above $\gamma_{1}$ lies in the affine hyperplane $\left[x_{2}=d\right]=A_{1}$ and joins $(d, d, 0)$ to $(-d, d, 0)$.

For each $1 \leq i \leq 4$ the side $f_{i}$ is parallel to the axis $x_{j(i)} j(i)=1,2$ $(1 \leq i \leq 4)$, which is one of the two $x$-axis. The $f_{1}$ just above, is parallel to the $x_{1}$-axis. We shall demand that $\gamma_{i}$ lies on the "side" of the affine hyperplane determined by

$$
A_{i} \cap\left[L_{j(i)}(y) \geq 0\right]=A_{i}^{+} .
$$

In the case of $\gamma_{1}$ we have thus

$$
\gamma_{1} \subset\left[x_{2}=d\right] \cap\left[L_{1}(y) \geq 0\right]=A_{1}^{+} .
$$

We shall join the four pieces $\gamma_{1} \cup \gamma_{2} \cup \gamma_{3} \cup \gamma_{4}=\gamma$ and obtain thus the required mapping of $\square_{d}^{2}$ in $G$. This mapping is piecewise smooth.

Observe that the above mapping is not a priori (1-1) and $\gamma$ is not necessarily an embedding. But that if $\alpha<0$, i.e. if $L_{1}, L_{2}$ have opposite signs then the above construction gives a Lip-embedding (i.e. an embedding by a bi-Lip mapping).

We shall further demand:

i) $\gamma$ is an $L L(d)-\square_{1}^{2} \subset G$ for the left invariant Riemannian structure of $G$.

ii) For each arc $\gamma_{i}$ the set

$$
\pi^{-1}(|y|<C) \cap \gamma_{i}=\gamma_{i}^{C},
$$


where $\pi: \mathbb{R}^{2} \bowtie V \longrightarrow V$ is the canonical projection, consists of exactly two straight line segments parallel to the $y$-axis emmunating from the two vertices. In the case of $\gamma_{1}$ we have $\gamma_{1}^{C}=\gamma_{1}^{+} \cup \gamma_{1}^{-}$where

$$
\gamma_{1}^{ \pm}=( \pm d, d, y), \quad 0<L_{1}(y)<C
$$

(not the same $C>0$ ).

Remark. For typographical reasons, I did not draw a picture. But a nice picture can be drawn and the reader should do so for himself ( $c f$. [5, Section 2.B]).

The only point that is not obvious in the construction is i). This will be verified by an explicit parametrization for the curve $\gamma_{1}$. The other pieces $\gamma_{2}, \gamma_{3}, \gamma_{4}$ can be treated analogously. To do that we identify $A_{1}$ with $P^{+}$, the upper half plane $y>0$, in the obvious way, and we set

$$
\begin{gathered}
\gamma(t)=(x(t) \cdot d, y(t)) \in P^{+}, \quad-1 \leq t \leq 1, \\
-1 \leq x(t) \leq 1, \quad 0 \leq y(t) \leq C \log d, \\
x( \pm 1)= \pm 1, \quad y( \pm 1)=0,
\end{gathered}
$$

where we impose the following additional conditions

$$
x( \pm t)= \pm 1, \quad|t| \in\left[1-2 c_{0}, 1\right]
$$

i.e. $x$ is constant near the end points of $[-1,1]$. In between i.e. $t \in$ $\left[-1+2 c_{0}, 1-2 c_{0}\right]$ the function $x(t)$ is $C^{\infty}$ and is close to being linear. We shall also assume $c_{0} \ll 1$.

$$
y(t)=C \log d, \quad t \in\left[-1+c_{0}, 1-c_{0}\right],
$$

for some large $C$ and the same $c_{0}$ as in (3.1.2). In the intervals $[-1,-1+$ $\left.c_{0}\right] \cup\left[1-c_{0}, 1\right], y(t)$ is $C^{\infty}$ and is monotone and almost linear. The constancy of $x$ near the end points guarantees that the condition ii) is verified. If the choice of $C$ in (3.1.3) is large enough then the condition i) will be verified. This is the only point where we have to make a (trivial) estimate. But this point is actually obvious and can be verified with the use of the orthonormal basis (2.4.1) of $T G$ constructed in 2.4.i). The details will be left to the reader who is strongly advised to do this 
and to compare it with the higher dimensional analogue (3.2.18) and with the Lip property in Section 3.2.3.

REMARK. When $\alpha<0$, i.e. when the group $G$ is a $C$-group ( $c f$. Section 0 ), the above construction actually gives $\gamma=\gamma_{1} \cup \gamma_{2} \cup \gamma_{3} \cup \gamma_{4}$ which is a $C^{\infty}$ embedded 1-dimensional sphere $S^{1}$ which, near the four vertices $( \pm d, \pm d, 0) \in \gamma$ reduces to the following four line segments, that are perpendicular to the $0 x_{1}, x_{2}$ coordinate plane

$[\xi \in \gamma, \operatorname{dist}(\xi,( \pm d, \pm d, 0) \leq C]=( \pm d, \pm d, 0)+[(0,0, y),-C \leq y \leq C]$

\subsubsection{A generalization: Groups of rank 1 .}

Let $Q=N \bowtie V$, where $V \cong \mathbb{R}$ is the real line and $N$ some simply connected nilpotent group (cf. 2.4.ii)). We shall assume that $g, h \in \mathfrak{n}$ (the Lie algebra of $N$ ) are two vectors that satisfy

$$
\begin{aligned}
|\operatorname{Ad}(y) g| & \leq C \exp (-\alpha y), & & y>0, \\
|\operatorname{Ad}(y) h| & \leq C \exp (-\beta y), & & y<0,
\end{aligned}
$$

where

$$
\alpha>0, \quad \beta<0,
$$

i.e. we shall assume that $Q$ is a $C$-group ( $c f$. Section 0 ), and that the action of $V$ on $N$ has two roots with real parts of opposite sign.

The nilpotency of $N$ implies that a high enough group commutator

$$
[\ldots,[X, Y], Y], \ldots, Y]=e=\text { Neutral element of } N, \quad X, Y \in N \text {. }
$$

If we multiply out that commutator, we obtain (a "universal relation in $N "$ )

$$
X^{p_{1}} Y^{q_{1}} X^{p_{2}} \cdots X^{p_{r}} Y^{q_{r}}=e, \quad X, Y \in N,
$$

where $r \geq 1, p_{1}, p_{2}, \ldots, q_{1}, q_{2}, \cdots \in \mathbb{Z}$ are fixed. This relation will allow us to embed in $N$ a polygonal (i.e. piecewise smooth) curve whose 
vertices will be

$$
\begin{aligned}
& P_{0}=e, \\
& P_{1}=X^{p_{1}} \\
& P_{2}=X^{p_{1}} Y^{q_{1}}, \cdots \\
& P_{2 j}=X^{p_{1}} Y^{q_{1}} \cdots X^{p_{j}} Y^{q_{j}}, \\
& P_{2 j+1}=X^{p_{1}} Y^{q_{1}} \cdots X^{p_{j+1}}, \quad j \geq 1 .
\end{aligned}
$$

The above pattern is clearly periodic no matter what $X, Y \in N$ is. We shall set

$$
X=\operatorname{Exp}(d g), \quad Y=\operatorname{Exp}(d h), \quad d>10^{10},
$$

with $g, h \in \mathfrak{n}$ as in (3.1.4), and shall join the successive vertices with pieces of one parameter subgroups:

- We join $P_{2 j}$ with $P_{2 j+1}$ with

$$
P_{2 j} \operatorname{Exp}\left(p_{j+1} g t\right), \quad 0<t<d .
$$

- We join $P_{2 j+1}$ with $P_{2 j+2}$ with

$$
P_{2 j+1} \operatorname{Exp}\left(q_{j+1} h t\right), \quad 0<t<d .
$$

One should observe that this construction is a direct generalization of the construction of the standard square $\square_{d}^{2}$ in $\mathbb{R}^{2}$, where the relation (3.1.6) is just the first commutator

$$
X Y X^{-1} Y^{-1}=0 \text {. }
$$

When $N=\mathbb{H}$ is the Heizenberg group $\left(c f .1 .2\right.$. vii $\left._{h}\right)$ ) an easy application of the Baker-Campbell-Hausdorff formula ( $c f$. [1]) shows that we can take

$$
X Y^{2} X Y^{-1} X^{-2} Y^{-1}=e,
$$

for a universal relation (cf. [5, Section $\left.5 . \mathrm{B}_{3}\right]$ ).

We can now generalize the construction of 3.1 .1 with $\square_{d}^{2} \subset \mathbb{R}^{2}$ replaced by the above $2 r$-sided polygon $P(d) \subset N$. Each side $f$ of $P(d)$ will be replaced by some smooth curve $\gamma_{i} \subset Q=N \bowtie V$ exactly 
as in (3.1.1). We start from the initial vertex of $f$ and while keeping the $N$-coordinates fixed, we dip in to depth - $C \log d$ in $V$ (i.e. the $y$ coordinate), and in the correct direction that is determined by (3.1.4), (3.1.5). Then we keep fixed the $V$-coordinate and cover the distance along $f$ in the $N$-coordinates. We then finally come back to $y=0$, in the $V$ coordinate, and to the second vertex of $f$ on $N$.

The estimate (2.4.2), (3.1.4), (3.1.5) allows us then to make sure that the new polygon $P^{*}(d) \subset N \bowtie V \subset Q$ is an $L L(d)-\partial \square_{1}^{2} \subset Q$. This is the analog of the condition 3.1.1.i). The (C)-condition (3.1.5), together with the way we made the construction allows us to guarantee that the analog of the Remark 3.1.1 holds for this polygonal curve.

The above $P^{*}(d)$ can be chosen, just as in Remark 3.1.1, to be a $C^{\infty}$-embedding of a 1-dimensional sphere.

Observe that in certain cases the $2 r$ points of (3.1.7) may not be distinct. The above construction should then be made on a shorter periodic subpatern of (3.1.7). At any rate the only case where we shall use the above construction is when $N$ is a group of "Heizenberg type", i.e. for the relation (3.1.8). In that case the description of the above construction simplifies (cf. [5]).

\subsection{The First Basic Construction.}

\subsubsection{Notations and definitions.}

i) We shall consider here the group $G=\mathbb{R}^{r} \bowtie V=\mathbb{R}^{r} \bowtie \mathbb{R}^{s}$ of 2.4.i) and we shall use the exponential coordinates $\left(x_{1}, \cdots x_{r} ; y_{1}, \cdots y_{s}\right)$ $\in \mathbb{R}^{r+s}$ and the orthonormal basis (2.4.1) of $T G$ defined in 2.4.i).

It will be convenient to use these coordinates to identify $G$ with $\mathbb{R}^{r+s}$, and to use "+" to indicate the Euclidean addition in $\mathbb{R}^{r+s}$. Observe, however, that then

$$
x+g=x \cdot g \in G, \quad x \in \mathbb{R}^{r}, g \in G,
$$

where "." indicates the multiplication in $G$. We shall denote by

$$
\pi_{V}: G \longrightarrow V, \quad \pi_{R}: G \longrightarrow \mathbb{R}^{r},
$$

the canonical Euclidean projections induced by the identification of $G=\mathbb{R}^{r} \times V$. This identification induces an identification

$$
T G=T \mathbb{R}^{r} \oplus T V,
$$


where the sum is orthogonal for the left invariant Riemannian structure on $T G$. Furthermore, on $T V$ the Riemannian scalar product coincides with the Euclidean one. On $T \mathbb{R}^{r}$ the Riemannian and the Euclidean norms

$$
|\xi|_{G},|\xi|_{\text {Euc }}, \quad \xi \in T \mathbb{R}^{r} \subset T_{(x, y)} G,
$$

can be negotiated with the help of the basis (2.4.1). In particular, it is clear that if

$$
\xi \in \operatorname{Vec}\left(\frac{\partial}{\partial x_{i_{1}}}, \ldots, \frac{\partial}{\partial x_{i_{a}}}\right)
$$

lies in some coordinate subspace of $\mathbb{R}^{r}$ we have

$$
|\xi|_{G} \leq C|\xi|_{\text {Euc }} \sup _{1 \leq j \leq a}\left(\exp \left(-L_{i_{j}}(y)\right)\right.
$$

ii) Let

$$
\square_{d}^{r}=\left\{\left(x_{1}, \ldots x_{r}\right) \in \mathbb{R}^{r}:\left|x_{j}\right| \leq d, j=1, \ldots r\right\}
$$

be as in Section 3.0. Let $I=\left(i_{1}, \ldots, i_{s}\right) \subset(1, \ldots, r)$ be a subset and let $J=(1, \ldots, r) \backslash I,(|I|=s,|J|=r-s)$. Let further $\varepsilon=\left\{\varepsilon_{j}\right\}_{j=1}^{r}$ be such that

$$
\varepsilon_{j}= \pm d, \quad j \in J, \quad \varepsilon_{i}=0, \quad i \in I
$$

We shall denote then

$$
F=F(I ; \varepsilon)=\left\{x \in \mathbb{R}^{r}:\left|x_{i}\right| \leq d, i \in I, x_{j}=\varepsilon_{j}, j \in J\right\} \subset \square_{d}^{r},
$$

which is one of the $2^{r-s} s$-dimensional faces of $\square_{d}^{r}$. If we define the slices of $\square_{d}^{r}$ by

$$
F_{I}=\left\{x \in \mathbb{R}^{r}:\left|x_{i}\right| \leq d, i \in I, x_{j}=0, j \in J\right\},
$$

we clearly have with obvious notations

$$
F=F(I ; \varepsilon)=F_{I}+\varepsilon
$$

For $F$ as above we shall denote by $\xi_{F} \in F$ the center of that face. 
The 0-dimensional faces are the vertices of $\square_{d}^{r}$. More generally, we denote by $\partial_{s} \square_{d}^{r}$ the union of all the above $s$-dimensional faces so that

$$
\partial_{0} \square_{d}^{r} \subseteq \partial_{1} \square_{d}^{r} \subseteq \cdots \partial_{r-1} \square_{d}^{r}=\partial \square_{d}^{r} .
$$

For every face $F=F(I ; \varepsilon)$ as above we shall denote as usual

$$
\partial F=\cup\left[F\left(J ; \varepsilon^{\prime}\right), J \subset I, I \neq J, F\left(J ; \varepsilon^{\prime}\right) \subset F\right] .
$$

The slice $F_{I}$ with $|I|=s$ can be identified with $\square_{d}^{s}$, the boundary $\partial F_{I}$ is then the $\partial \square_{d}^{s}$ that corresponds in that identification.

iii) We shall assume that the $L_{1}, \ldots, L_{r} \in V^{*}$ that we used in the definition of the group $G=\mathbb{R}^{r} \bowtie V$ (cf. 2.4.i)) are all non zero and satisfy the following condition:

For every $I \subset[1, \ldots, r],|I| \leq r-1$ the set $\left(L_{i}, i \in I\right) \subset V^{*}$ is $\mathrm{NC}$ (cf. Section 1.1.2).

We shall identify, once and for all, $V \simeq V^{*}$ by some fixed scalar product on $V$. We shall also fix for each $\varnothing \neq I \subset[1, \ldots, r],|I| \leq r-1$ (cf. 1.1.5)

$$
\zeta_{I}=\sum_{i \in I} \lambda_{i} L_{i},\left|\zeta_{I}\right|_{V} \leq C \log d, L_{i}\left(\zeta_{I}\right) \geq C \log d
$$

and $\lambda_{i}>0, i \in I$. It is important to observe that with the above definition, for any choice of $I_{1}, \ldots, I_{a}(a \geq 1)$ as in (3.2.4), we have

$$
\begin{aligned}
& L_{j}(\zeta) \geq C \log d, j \in \bigcap_{k=1}^{a} I_{k}, \\
& \zeta \in \text { Convex Hull }\left[\zeta_{I_{k}}, 1 \leq k \leq a\right] .
\end{aligned}
$$

\subsubsection{The Auxilliary Construction.}

We shall consider now $F=F(I ; \varepsilon),|I|=r-1$ some $r-1$ dimensional face of $\square_{d}^{r}$ and we shall fix a decreasing sequence of subfaces

$$
F=F_{r-1} \supseteq F_{r-2} \supseteq \cdots \supseteq F_{0}
$$

such that $\operatorname{dim} F_{j}=j=\left|I_{j}\right|$, where

$$
I=I_{r-1} \supset I_{r-2} \supset \cdots
$$


is the decreasing sequence of multiindices that correspond to these subfaces. We shall denote also ( $c f$. Section 3.2.1)

$$
\begin{gathered}
\xi_{j}=\xi_{F_{j}}, \quad 0 \leq j \leq r-1, \quad F_{0}=\left\{\xi_{0}\right\}, \\
\zeta_{j}=\zeta_{I_{j}}, \quad 1 \leq j \leq r-1 .
\end{gathered}
$$

We shall fix

$$
0 \leq \alpha_{j}, \quad \beta_{j} \leq 1, \quad 1 \leq j \leq r-1,
$$

and we shall define inductively

$$
x_{0}=\xi_{0}, \quad x_{j+1}=\left(1-\alpha_{j+1}\right) \xi_{j+1}+\alpha_{j+1} x_{j} \in F_{j+1},
$$

where $j=0,1, \ldots, r-2$, and

$$
y_{0}=0, \quad y_{j+1}=\left(1-\beta_{j+1}\right) \zeta_{j+1}+\beta_{j+1} y_{j} \in V,
$$

where $j=0,1, \ldots, r-2$. We shall also define

$\Phi_{j}=\left(x_{j}, y_{j}\right) \in F_{j} \times V \subseteq \square_{d}^{r} \times V \subset \mathbb{R}^{r} \times V=G, \quad j=0, \ldots, r-1$

Let us now define the following functions of $0 \leq \theta \leq 1$

$$
\alpha(\theta)= \begin{cases}0, & \theta \in[0, c], \\ 1, & \theta \in[1-3 c, 1], \\ C^{\infty}, & \text { increasing, and almost linear in between } .\end{cases}
$$

$$
\beta(\theta)= \begin{cases}0, & \theta \in[0,1-2 c] \\ 1, & \theta \in[1-c, 1], \\ C^{\infty}, & \text { and increasing in between } .\end{cases}
$$

The choice of $0<c \ll 1$ is irrelevant as long as it is small enough. What counts in the above definition are the following facts

$$
\begin{aligned}
& |d \alpha|,|d \beta| \leq C, \text { and } \beta(\theta) \neq 0, \theta \in[0,1], \\
& \text { implies } \alpha\left(\theta^{\prime}\right)=1, \theta^{\prime} \in[\theta-c, \theta+c] .
\end{aligned}
$$

In the inductive construction (3.2.6), (3.2.7), I shall then set

$$
\alpha_{j}=\alpha\left(\theta_{j}\right), \quad \beta_{j}=\beta\left(\theta_{j}\right), \quad j=1, \ldots, r-1,
$$


where $\theta=\left(\theta_{1}, \ldots, \theta_{r-1}\right) \in[0,1]^{r-1}$. We obtain thus

$$
\begin{aligned}
& \Phi_{r-1}:[0,1]^{r-1} \longrightarrow G \\
& \Phi_{r-1}\left(\theta_{1}, \ldots, \theta_{r-1}\right) \\
& \quad=\left(x_{r-1}\left(\theta_{1}, \ldots, \theta_{r-1}\right), y_{r-1}\left(\theta_{1}, \ldots, \theta_{r-1}\right)\right)
\end{aligned}
$$

We clearly have ( $c f .(3.2 .1))$

$$
\begin{aligned}
d x_{r-1} & =d \pi_{R} \circ d \Phi_{r-1}: T[0,1]^{r-1} \longrightarrow T \mathbb{R}^{r} \\
d y_{r-1} & =d \pi_{V} \circ d \Phi_{r-1}: T[0,1]^{r-1} \longrightarrow T V
\end{aligned}
$$

If we norm $T \mathbb{R}^{r}$ in (3.2.11) with the Euclidean norm ( $c f$. 3.2.1.i)) we obtain by the definition (3.2.6) that

$$
\left\|d \pi_{R} \circ d \Phi_{r-1}\right\|_{\text {Euc. }} \leq C d
$$

and we obtain also (cf. (3.2.4))

$$
\left\|d \pi_{V} \circ d \Phi_{r-1}\right\| \leq C(\log d)
$$

where on $T V$ in (3.2.14) we can assign either the Euclidean or the Riemannian norm because these two norms coincide. Both (3.2.13) (3.2.14) can trivially be verified by induction. In fact we can improve upon (3.2.13). Towards that we distinguish two cases:

Case 1. $\theta \in[0,1]^{r-1}$ is such that $\beta_{j}(\theta) \neq 0, j=1, \ldots, r-1$. By (3.2.9) it follows then that

$$
d x_{r-1}=0 .
$$

Case 2. $\theta \in[0,1]^{r-1}$ is such that there exists some $1 \leq p \leq r-1$ such that $\beta_{p}=0$. From the definition (3.2.7) it follows then that

$$
y_{r-1} \in \text { Convex Hull }\left[\zeta_{r-1}, \ldots, \zeta_{p}\right] \text {. }
$$

We shall choose the largest possible $p$ so that $\beta=0$ and either

i) $p=r-1$.

ii) $p<r-1, \beta_{p+1} \neq 0, \ldots, \beta_{r-1} \neq 0$. 
In both cases the definition (3.2.6) together with (2.2.9) implies that

$$
d x_{r-1}\left(T[0,1]^{r-1}\right) \subset\left\{\frac{\partial}{\partial x_{i}}: i \in I_{p}\right\} .
$$

But this together with (3.2.2), (3.2.3), (3.2.5), (3.2.13), (3.2.16) implies that if the $C$ in (3.2.4) is large enough, the norm satisfies:

$$
\left\|d x_{r-1}\right\|_{G} \leq C d \exp (-C \log d) \leq C,
$$

where $\|\cdot\|_{G}$ means that we assign now $T \mathbb{R}^{r}$ in (3.2.11) with the left invariant Riemannian norm of $G$. Putting together (3.2.15), (3.2.17), (3.2.14) and 3.2.1.i). We conclude, that for an appropriate choice of the constants $C>0$ in (3.2.4), we have

$$
\Phi_{r-1}:[0,1]^{r-1} \longrightarrow G, \quad\left\|d \Phi_{r-1}\right\| \leq C \log d,
$$

where, of course, we put the Euclidean norms on $T[0,1]^{r-1}$ and the left invariant Riemannian norm on $T G$.

\subsubsection{The Extension Operator and the Construction.}

The notation $F_{I}, I \subset[1,2, \ldots, r]$ for the various slices of the cube $\square^{r}$ that were introduced in 3.2.1.ii) will be preserved here, with the additional convention that I shall use the same notation

$$
F_{I} \subset \square_{1}^{r}, \quad F_{I} \subset \square_{d}^{r},
$$

to indicate the corresponding slice, for some fixed $I$ (i.e. $x_{j}=0, j \notin I$ ) for the unit cube and the $d$-cube.

I shall consider throughout in this section and in the next, mappings

$$
\Phi: \square_{1}^{r} \longrightarrow \square_{d}^{r} \times V
$$

where $\operatorname{Dom}(\Phi) \subset \square_{1}^{r}$ is some subset of $\square_{1}^{r}$. More precisely, we shall consider

$$
\begin{aligned}
& f: \partial F_{I} \longrightarrow F_{I} \times V \subset G, \\
& E f: F_{I} \longrightarrow F_{I} \times V \subset G,
\end{aligned}
$$


where $G$ in (3.2.20), (3.2.21) is as in 3.2.1.i). $\partial F_{I}, F_{I}$ in (3.2.20), (3.2.21) on the left hand side refer to the unit cube $\square_{1}^{r}$ while on the right hand side refer to the same slices in $\square_{d}^{r}$.

In this section I shall explain first how, given a Lip-mapping as in (3.2.20) for some $I$, we can extend it to a Lip-mapping $E f$ as in (3.2.21) by some specific extension operator $E=E_{I}$, such that

$$
\left.E f\right|_{\partial F_{I}}=f .
$$

This is done as follows:

Let $x \in F_{I} \subset \square_{1}^{r}, I \neq \varnothing$, then we can write (essentially) uniquely

$$
x=(1-\theta) \xi_{I}+\theta y, \quad 0 \leq \theta \leq 1, \quad y \in \partial F_{I},
$$

where $\xi_{I}=\left(\right.$ Center of $\left.F_{I}\right)=0$. We shall then define

$E f(x)=\left(\left(1-\alpha_{I}(\theta)\right) \xi_{I}+\alpha_{I}(\theta) f_{F}(y), \quad\left(1-\beta_{I}(\theta)\right) \zeta_{I}+\beta_{I}(\theta) f_{V}(y)\right)$,

where

$$
f=\left(f_{F}, f_{V}\right) \in F_{I} \times V,
$$

are the two coordinate functions, and where

$$
\begin{aligned}
& 0 \leq \alpha_{I}(\theta), \beta_{I}(\theta) \leq 1, \\
& \alpha_{I}(0)=\beta_{I}(0)=0, \\
& \alpha_{I}(1)=\beta_{I}(1)=1,
\end{aligned}
$$

are nondecreasing functions that satisfy the additional properties (3.2.8) (and which in fact can be taken to be independent of $I$ ). The $\xi_{I} \in V$ will be chosen to be as in (3.2.4).

The inductive construction. We shall now construct Lip mappings $\varphi_{I}, I \subset[1,2, \ldots, r],|I| \leq r-1$ and $\varphi_{s}, s=0,1, \ldots, r-1$ that have the following properties:

i) $\varphi_{I}: F_{I} \longrightarrow F_{I} \times V \subset G$ as in (3.2.19).

ii) $\varphi_{\varnothing} \equiv e \in G$ (the neutral element).

iii) $\varphi_{s}: \partial_{s} \square_{1}^{r} \rightarrow \partial_{s} \square_{d}^{r} \times V \subset G, s=0, \ldots, r-1$, with the notations of 3.2.1.ii) and $\varphi_{0}$ is the identity mapping scaled by $d$.

iv) If $I \subset[1,2, \ldots, r],|I|=s, \varepsilon=\left(\varepsilon_{1}, \ldots, \varepsilon_{r}\right), \varepsilon_{j}=0, \pm 1$ and $\varepsilon+F_{I}=F(I ; \varepsilon)$ as in 3.2.1.ii) then, $\varphi_{s}(\varepsilon+x)=\varepsilon d+\varphi_{I}(x), x \in F_{I}$. 
The construction is done by induction as follows: ii), iii) and iv) determine $\varphi_{0}$ inambiguously. Assume that for some $0 \leq s \leq r-1, \varphi_{s}$ and $\varphi_{I},|I| \leq s$ have been defined and satisfy i)-iv). Let $I \subset[1,2, \ldots, r]$ be such that $|I|=s+1<r$. Then $F_{I}$ can be identified to $F \subset \partial_{s+1} \square_{1}^{r}$ some face of $\square_{1}^{r}$. The choice of that $F$ is in general not unique. This identification identifies $\partial F_{I}$ to $\partial F$ and defines

$$
\left.f=-\varepsilon d+\left.\varphi_{s}\right|_{\partial F}: \partial F_{I} \longrightarrow F_{I} \times V \text { (for the appropriate } \varepsilon\right) .
$$

This definition is inambiguously because of the inductive hypothesis. We shall define then $\varphi_{I}=E_{I} f$ by applying the extension operator. We shall then define $\varphi_{s+1}$ by demanding that iv) should hold. This is clearly possible and $\left.\varphi_{s+1}\right|_{\partial_{s} \square^{r}}=\varphi_{s}$ is an extension of $\varphi_{s}$.

The final step of this construction is a mapping

$$
\varphi=\varphi_{r-1}: \partial \square_{1}^{r} \longrightarrow \partial \square_{d}^{r} \times V \subset G .
$$

Let

$$
S=\varphi\left(\partial \square^{r}\right) \subset \mathbb{R}^{r} \bowtie V \subset G
$$

We shall show that it is possible to make the above constructions in such a way that $\varphi$ and $S$ in (3.2.23), (3.2.24) have the following properties:

(Lip) Lipschitz property. In (3.2.23) we have $\varphi \in L L(d)$ and $S$ is an $L L(d)-\partial \square_{1}^{r} \subset G$.

\section{(Trans) Transversality properties.}

Trans i). There exists $0<c \ll 1$ such that for every vertex $P \in \partial \square_{1}^{r}$, $B_{c}(P)$, the $c$-neighbourhood of $P$, is mapped into $\{P\} \times V \subset \partial \square_{d}^{r} \times$ $V \subset G$, where $P$ is identified to the corresponding vertex of $\partial \square_{d}^{r}$. Furthermore, if $F=F(I ; \varepsilon) \subset \partial \square_{1}^{r}$ is some $(r-1)$-dimensional face of $\square_{1}^{r}$ such that $\partial_{0} \square_{1}^{r} \ni P \in F$, then $\left.\varphi\right|_{F \cap B_{c}(P)}=\varphi_{P, F}$ satisfies

$$
\{P\} \times C_{I} \supset \text { Image }\left(\varphi_{P, F}\right) \supset\{P\} \times\left(C_{I} \cap V_{C}\right),
$$

for some $C>0$, where

$$
V_{C}=[u \in V,|u| \leq C], \quad C_{I}=\left(\sum_{i \in I} \lambda_{i} L_{i}, \lambda_{i} \geq 0, i \in I\right)
$$


What the above says in words is simply this: The vertices of $\square_{1}^{r}$ go to the vertices of $\square_{d}^{r}$, and near each vertex $P$ of $\square_{1}^{r}$, the various faces $F(I, \varepsilon)$ that contain $P$, go "nicely" to the tips of the corresponding cones $C_{I}$ that stick out of $\varphi(P)$.

Trans ii). For appropriate $C, c>0$, we have

$$
\xi \in \partial \square_{1}^{r}, \quad|\pi \circ \varphi(\xi)|_{V} \leq C
$$

implies that there exists $P \in \partial_{0} \square_{1}^{r}$ such that $\xi \in B_{c}(P)$,

where $\pi=G \longrightarrow V$ is the canonical projection. In words: Unless $\xi$ is near a vertex $P \in \partial_{o} \square_{1}^{r}$, its image $\varphi(\xi)$ in $G$ lies far away from $\mathbb{R}^{r}$.

With the "additive" notations of 3.2.1.i) and the notations of Trans.i) and, an abusive but clear meaning of $\approx$, we can summarize

$$
\begin{gathered}
\varphi\left(F \cap B_{c}(P)\right) \approx \varphi(P)+\left(C_{I} \cap V_{C}\right), \quad P \in \partial_{0} \square_{1}^{r}, \\
S \cap \pi^{-1}[u \in V:|u| \leq C] \subset \varphi\left(\partial_{0} \square_{1}^{r}\right)+V_{C} .
\end{gathered}
$$

At this point, we should observe that once $P \in \partial_{0} \square_{1}^{r}$ has been fixed, for every $I \subset[1,2, \ldots, r]$ with $|I|=r-1$, there is exactly one face $F=F(I, \varepsilon)$ such that $P \in F$. It follows that the above gives a nice description of how the neighbourhood of every vertex in $\square_{1}^{r}$ is mapped into $G$.

The special case of the $C$-condition. Let us go back to 3.2.1.iii) and let us denote by $V_{2}=\operatorname{Vec}\left(L_{1}, \ldots, L_{r}\right) \subset V$. Let us also assume that the vectors $L_{1}, \ldots, L_{r}$ are the vertices of some simplex and $0 \in$ Int $\left[L_{1}, \ldots, L_{r}\right]=\operatorname{Int} \sigma$. In this case just by looking at the simplex $\sigma$ "around $0 "$ we see

$$
\left[C_{I} \subset V_{2}: I \subset[1,2, \ldots, r]:|I|=r-1\right]
$$

is a tacelation of $V_{2}$ (i.e. $\stackrel{\circ}{C}_{I} \cap \stackrel{\circ}{C}_{J}=\varnothing, I \neq J, \cup C_{I}=V_{2}$ ). From this, and the Transversality conditions that $S$ satisfies, we see that under the $C$-condition we have

$$
\varphi\left(\partial \square_{1}^{r} \cap B_{c}(P)\right) \approx \varphi(P)+\left[u \in V_{2}:|u| \leq C\right], P \in \partial_{0} \square_{1}^{r}
$$

with the obvious use (or rather abuse) of the notations. 
In fact, under the above $C$-condition for the set $L_{1}, \ldots, L_{r} \in V^{*}$, we can avoid altogether the use of the extraneous scalar product on $V$ which was essential in the above construction $(c f$. (3.2.4)). Indeed we can consider then $V_{2} \subset V$ some (linear) direct complement of $V_{1}=\{x$ : $\left.L_{j}(x)=0,1 \leq j \leq r\right\} \subset V$ and fix $\sigma=\left[x_{1}, \ldots, x_{r}\right] \subset V_{2}$ some simplex such that $0 \in \operatorname{Int}(\sigma)$ and $L_{i}\left(x_{j}\right)>0(i, j=1, \ldots, n, i \neq j)$. Instead of using 1.1 .5 we can then define the $\zeta_{I}$ 's in $(3.2 .4)$ by

$$
\zeta_{I}=\log d\left(\sum_{i \notin I} x_{i}\right)
$$

The rest of the argument works as before, and the only difference is that the simplex $\sigma=\left[x_{1}, \ldots, x_{r}\right]$ is not necessarily the simplex $\left[L_{1}, \ldots, L_{r}\right]$ that we used in (3.2.29).

It is of some interest to observe that in the above case we can even make the set $S$ of (3.3.24) homeomorphic to an $r-1$ sphere. Indeed the only thing that stops the mapping $\varphi$ that defines $S$ from being (1-1), is the fact that the functions $\alpha(\theta)$ and $\beta(\theta)$ that were used have common intervals of constancy ( $c f$. (3.2.8)). This point can easily be rectified, and yet preserve all the other properties of $\alpha, \beta$ (especially (3.2.18)) that are needed for the construction.

Proof of the Lipschitz properties. The proof of the property $\varphi \in$ $L L(d)$ depends on a finite decomposition

$$
\bigcup_{\alpha=1}^{A} \bar{\Omega}_{\alpha}=\partial \square_{1}^{r}
$$

where $\Omega_{\alpha}$ is a relative open set. The sets $\Omega_{\alpha}$ are constructed as follows. Let $x \in \partial \square_{1}^{r}$. Then as long as $x$ lies outside the union of finitely many affine subspaces we can write uniquely

$$
\begin{gathered}
x=x_{r-1}=\left(1-\theta_{r-1}\right) \xi_{F_{r-1}}+\theta_{r-1} x_{r-2}, \\
F_{r-1} \subset \partial_{r-1} \square_{1}^{r}, \quad 0<\theta_{r-1}<1, \quad x_{r-2} \in \partial_{r-2} \square_{1}^{r},
\end{gathered}
$$

where $F_{r-1}$ is an $(r-1)$-dimensional face uniquely determined by $x$. Furthermore, the mapping

$$
x_{r-1} \longrightarrow\left(\theta_{r-1}, x_{r-2}\right), \quad \theta_{r-1} \geq c,
$$


is Lip $C$ for any $c>0$ and $C=C(c)$. This process can be iterated, and if we assume that $x_{r-2}$ avoids a finite number of affine subspaces we can write

$$
\begin{aligned}
& x_{r-2}=\left(1-\theta_{r-2}\right) \xi_{F_{r-2}}+\theta_{r-2} x_{r-3}, \quad F_{r-2} \subset \partial_{r-2} \square_{1}^{r}, \\
& 0<\theta_{r-2}<1, \quad x_{r-3} \in \partial_{r-3} \square_{1}^{r},
\end{aligned}
$$

and so on.

It follows that with the exception of $\mathcal{E}$, an exceptional subset of $\partial \square_{1}^{r}$ which is the union of finitely many affine pieces of dimension at most $r-2$, we can determine uniquely for every $x \in \partial \square_{1}^{r}$ a sequence

$$
\mathcal{F}(x): F_{r-1} \supset F_{r-2} \supset \cdots \supset F_{0}
$$

of faces $F_{j}$ of $\square_{1}^{r}, \operatorname{dim} F_{j}=j$ and a vector

$$
\theta(x)=\left(\theta_{r-1}(x), \ldots, \theta_{1}(x)\right) \in[0,1]^{r-1}
$$

such that for every $r-1 \geq a \geq 1$, the mappings

$$
x \longmapsto \theta_{j}(x), \quad r-1 \geq j \geq a,
$$

are $\operatorname{Lip}(C)$ as long as we stay away from $\theta_{j}=0(r-1 \geq j \geq a+1)$. The open subsets $\Omega_{\alpha}(3.2 .31)$ are then determined by requiring that

$$
x \notin \mathcal{E}, \quad \mathcal{F}(x) \text { is fixed } .
$$

By our constructions the mapping $\varphi$ of (3.2.23), (3.2.24) coincides on each $\Omega_{\alpha}$ with the mapping $\Phi_{r-1}$ constructed in (3.2.10), composed with the mapping $(3.2 .33),(3.2 .34)$. If we recall that both $\alpha(\cdot)$ and $\beta(\cdot)$ are equal to 0 in some neighbourhood of 0 , we see that the Lip property of $\varphi$ follows immediately from the above and (3.2.18). Indeed, as in the end of 3.2.2, if for some $x \in \Omega_{\alpha}$ this is a first $\theta_{r-k}$ (in the succesive construction $\left.\theta_{r-1}, \ldots\right)$ that is small enough, then $x_{r-k}$ lies in some small neighbourhood of $\xi_{F_{r-k}}$ in $\Omega_{\alpha} \cap F_{r-k}$ which is mapped on the fixed point $\left(\xi_{r-k}, \zeta_{r-k}\right)$ of Section 3.2.2. It follows that in the differential $d \varphi$ at $x$ only the coordinates $\theta_{r-1}, \ldots, \theta_{r-k+1}>c$ are involved.

Proof of the transversality property. To prove the transversality properties of $\varphi$ it suffices to prove that $\left.\varphi\right|_{\partial_{s} \square_{1}^{r}}=\varphi_{s}$ satisfies the corresponding properties and in particular (3.2.25)-(3.2.28) for $s=$ 
$0,1, \ldots, r-1$. For $s=0$ this is evident. The general case $s \geq 0$ follows then immediately by induction and the fact that $\alpha(\theta)=1$, $\theta \in[1-3 c, 1]$ while the $\beta(\theta)$ goes through the whole of its variation in the interval $[1-3 c, 1]$. In words, the above says that near the distinguished boundary $\partial_{r-2} \square_{1}^{r}$, and in particular near the vertices, $\varphi$ does not start moving in the $\mathbb{R}^{r}$ direction before we are already quite deep in the appropriate cone $C_{I}$ (and therefore already out of $V_{C}$ ).

Additional smoothness properties. When the $C$ condition is verified as in (3.2.29) and (3.2.30) we can guarantee that in the above construction $S=\varphi\left(\partial \square^{r}\right)$ defined in (3.2.24) is a $C^{\infty}$ embedded $(r-1)$ dimensional sphere $S^{r-1} \subset G$. This condition is not difficult to build in the above construction. The only difficulty lies in choosing the correct notations that tend to get out of hand. This was seen in the 1-dimensional case in Section 3.1.1 where we "negociate the corners" of the square by the local constancy of $\alpha$ near $\theta \sim 1$. Since no essential use will be made of this smoothness property the details will be omitted.

It should be noted, however, that there is another way of guaranteeing that smoothness by an "a posteriori" argument. What one can do, is start by the transversality conditions (Trans.) and use convolution to smooth out $\varphi$, and yet preserve the transversality conditions. Any pretence of global injectivity (i.e. the (1-1) property of $\varphi$ ) is, of course, lost with this operation. We can then use the Whitney perturbation technique [14], as explained in Section 4.5 to obtain an $S$ that is a $C^{\infty}(r-1)$-dimensional sphere in $G$, and still has the properties (Lip.) and (Trans.) described above.

\subsubsection{The Embedding of $S$ in the eigenvalue group.}

Let $N_{E} \subset N$ be the eigenvalue group as in Section 2.2 that corresponds to the eigenvalue algebra $\mathfrak{e}$ as in Section 2.1. All the notations and definitions of Section 2 will be preserved and we shall distinguish two cases:

$N_{E}$ is abelian. We shall then assume that $L_{1}, \ldots, L_{r}$ are the vertices of a simplex as in (2.1.2), and we shall fix the basis $e_{1}, \ldots, e_{q}$ in $\mathfrak{e}$, as in (1.3.7), so that $e_{i_{1}}, e_{i_{2}} \ldots e_{i_{r}}$ each lies in different root space.

We shall now use the notation of sections 2.1 and 2 and consider the Riemannian structure induced on $N_{E} \times V_{2}$ by $\langle\cdot, \cdot\rangle_{\bowtie}$ as in Section 2.3. 
This structure is a Riemannian structure of the kind defined in 2.4.i). The exponential coordinates of Section 2.1 can then be used (by the subnormality of the generated subalgebra the vectors $e_{i_{1}}, \ldots, e_{i_{r}}$ can be taken to be the first $r$ vectors of the basis (2.1.1)) to identify $\mathbb{R}^{r-1}$ with $V_{2}$ and $\mathbb{R}^{r}=\operatorname{Vec}\left(e_{i_{1}}, \ldots, e_{i_{r}}\right)$ with a submanifold of $N_{E}$. This identifies $\mathbb{R}^{r} \times \mathbb{R}^{r-1}$ with a submanifold of $N_{E} \times V_{2}$. Even in the split case ( $c f$. Section 2.2) when $N_{E} \times V_{2}$ is a subgroup, the above submanifold is not in general a subgroup (because of the "complex rotations" comming from the imaginary part of the roots). It is, however, clear from 1.2.viii), (1.3.4) that this manifold with the induced Riemannian structure is isometric to the Riemannian manifold defined in 2.4.i).

From this it follows that the $S=\varphi\left(\partial \square_{1}^{r}\right)$ defined in (3.2.24) can be embedded and thus be identified to a subset of $N_{E} \times V_{2} . S$ is in particular an $L L(d)-\partial \square_{1}^{r}$.

$N_{E}$ is of Heizenberg type. We then shall use the construction of Section 3.1.2 and the relation (3.1.8) in the group generated by the algebra (1.2.13), with $e \in H_{\alpha}, h \in H_{-\alpha}$ (with the notations of Section 3.1.2 and (1.2.13)). The considerations of 2.4.ii) apply then and we see that the polygon $P^{*}(d)=S$ constructed in Section 3.1.2 can be made to have the analogous properties (Lip.) and (Trans.: here we already are in the $C$-condition case). The analog of (3.2.30) therefore holds. This now reads as follows:

Near each of the six vertices $P_{i}(1 \leq i \leq 6), S$ is identical to

$$
P_{i} \cdot\left[u \in V_{2}:|u| \leq C\right]
$$

where "." indicates the group product in $N_{E} \bowtie V_{2}$ which is always a group ( $c f$. end of 2.2). We have thus embedded $S \subset N_{E} \times V_{2}$, with $\operatorname{dim} V_{2}=1$. The only difference with the previous abelian case is that now, globally, $S$ "lives" (spills out if you prefer) in all the coordinates of $N_{E} \bowtie V_{2}$. S is in particular an $L L(d)-\partial \square_{1}^{r}$ where now $r=2$.

\subsection{The Second Basic Construction.}

\subsubsection{Filling in a small cylinder.}

We shall use throughout the identification and the notations of 
Section 2.2, (2.2.2), (2.2.3)

$$
Q=N \times V, \quad V=V_{1} \times V_{2},
$$

$$
S \subset N_{E} \times V_{2}, \quad N_{E} \times V_{2} \subset\left(N \times V_{2}\right) \times V_{1}=Q,
$$

where $S$ is the $L L(d)-\partial \square_{1}^{r} \subset N_{E} \bowtie V_{2}$ constructed in (3.2.23), (3.2.24) and Section 3.2.4. $N_{E} \bowtie V_{2}$ is the group structure on $N_{E} \times V_{2}$ defined in Section 2.3. When $Q$ is split all the manifold products in (3.3.1), (3.3.2) are just group skew products. I shall also suppose, as I may, that the neutral element $e \in G$ lies on $S$.

In this section I shall assume throughout that $Q$ admits the property $\mathcal{F}$ (cf. Section 3.0) or at least the properties $\mathcal{F}_{p}$ for the relevant $p=2, \ldots, \operatorname{dim} Q-1$.

i) The 1-dimensional cylinder. The split case. Let $a, b \in V_{1}$, and $\ell=[a, b]$ the affine segment that joins these two points and let

$$
S \times \ell \subset\left(N_{E} \bowtie V_{2}\right) \bowtie V_{1} \subset Q .
$$

$S \times \ell$ is the lateral boundary of a hollow cylinder (It looks like an empty food can with top and bottom removed).

1) Filling the top and bottom. By the Lemma 2.3.1 we have

$$
S \times\{a\}, \quad S \times\{b\} \text { are } L L(d)-\partial \square_{1}^{r} \subset Q .
$$

By the property $\mathcal{F}_{r}$ we can "fill these in" and find

$$
\begin{gathered}
B_{a}, B_{b} \text { two } L L(d)-\square_{1}^{r} \subset Q, \\
\partial B_{a}=S \times\{a\}, \quad \partial B_{b}=S \times\{b\} .
\end{gathered}
$$

By Lemma 2.3.2 there exists

$$
\left\{\begin{array}{l}
\Phi \in L L(d), \\
\Phi: \partial \square_{1}^{r} \times[a, b] \longrightarrow Q, \quad \operatorname{Im} \Phi=S \times[a, b],
\end{array}\right.
$$

where we assign on $\partial \square_{1}^{r} \times[a, b] \subset \mathbb{R}^{r+1}$ its natural distance. It follows that

$$
B_{a} \cup B_{b} \cup(S \times[a, b]) \text { is an } L L(d)-\partial\left(\square_{1}^{r} \times[a, b]\right) \subset Q \text {. }
$$


Therefore if we assume that

$$
|\ell|=|a-b| \leq 1
$$

we can rescale and

$$
B_{a} \cup B_{b} \cup(S \times[a, b]) \text { is an } L L(d)-\partial \square_{1}^{r+1} \subset Q \text {. }
$$

2) Filling the can. By the property $\mathcal{F}_{r+1}$ we can find

$$
\begin{gathered}
B \text { an } L L(d)-\square_{1}^{r+1} \subset Q, \\
\partial B=B_{a} \cup B_{b} \cup(S \times[a, b]) .
\end{gathered}
$$

It will be abusive but convenient to abbreviate the information contained in (3.3.4)-(3.3.11) by writing

$$
\partial B=\operatorname{Rim}(S \times[a, b]) .
$$

ii) The 1 dimensional cylinder. The general case. We shall adapt here the previous construction in the general case, i.e. when $Q$ is not split. The notations of the previous section will be preserved. Clearly it is only the use of the Lemma 2.3.1 and 2.3.2 that has to be modified. Observe first of all that the fact that

$$
S \text { is an } L L(d)-\partial \square_{1}^{r} \subset N_{E} \bowtie V_{2},
$$

and the fact that the canonical projection $N_{E} \times V_{2} \longrightarrow V_{2}$ is a group homomorphism (for the group structure $N_{E} \bowtie V_{2}$ ), and therefore Lip (1), implies that

$$
S \subset N_{E} \times\left[u \in V_{2},|u|_{V_{2}} \leq c(\log d)^{c}\right] \subset N_{E} \times V_{2}
$$

It follows that Lemma 2.3.1 can be used (for the non split case), and (3.3.4)-(3.3.6) of the previous construction are not altered. (3.3.7)(3.3.9) on the other hand have to be "handled with care". We shall denote by

$$
\Phi: \partial \square_{1}^{r} \longrightarrow S, \quad \Phi \in L L(d)
$$


for the distance on $S$ induced by $S \subset N_{E} \bowtie V_{2}$, and we shall assume that

$$
|b-a| \leq(|a|+|b|+10)^{-C}
$$

for some appropriate $C>0$. We shall also use the linear scaling:

$$
\Psi:[0,1] \longrightarrow[a, b]
$$

We can consider then the composition of maps

$$
\begin{aligned}
& \partial \square_{1}^{r} \times[0,1] \underset{\Phi \times \operatorname{Id}}{\longrightarrow} S \times[0,1] \underset{\operatorname{Id} \times \Psi}{\longrightarrow} S \times[a, b] \\
& \stackrel{\subset}{\longrightarrow}\left(N_{E} \bowtie V_{2}\right) \times V_{1} \underset{\Theta}{\stackrel{C}{\longrightarrow} Q},
\end{aligned}
$$

where in (3.3.17) we denote by $N_{E} \bowtie V_{2}$ the direct product $N_{E} \times V_{2}$ with the Riemannian structure induced by the group $N_{E} \bowtie V_{2}$ as in Section 2.3, and on $\left(N_{E} \bowtie V_{2}\right) \times V_{1}$ we give the product distance and product Riemannian structure. By the Lemma 2.3.2 and (3.3.14) we see that

$$
\begin{aligned}
& \left\|\left.d \Theta\right|_{T\left(N_{E} \times V_{2}\right)}\right\|=O\left((\log d)^{C}\right), \\
& \left\|\left.d \Theta\right|_{T V_{1}}\right\|=O\left((\log d)^{C}(|a|+|b|+10)^{C}\right) .
\end{aligned}
$$

Since on the other hand by (3.3.13), (3.3.16)

$$
\Phi \times \operatorname{Id} \in L L(d), \quad \Psi \in \operatorname{Lip}(|b-a|), \quad I \in \operatorname{Lip}(1),
$$

we conclude from (3.3.15), (3.3.18) that the composition of the maps in (3.3.17) is a map

$$
\partial \square_{1}^{r} \times[0,1] \longrightarrow Q \text { in } L L(d),
$$

with constants that are uniform in $a$ and $b$. This controls the third term in (3.3.9) as before and we can thus fill in the "can" as before and find $B$ that satisfies (3.3.10), (3.3.11), (3.3.12).

The 2-dimensional cylinder. The split case. Let $a_{i, j} \in V_{1} i, j=$ 1,2 be the four vertices of a parallelepiped

$$
L=\left[a_{1,1}, a_{1,2}, a_{2,2}, a_{2,1}\right] \subset V_{1},
$$


114 N. Th. VARopoulos

with sides parallel to the first two axes of $V_{1}$. Let

$$
\left[a_{i, 1}, a_{i, 2}\right]=\ell_{i}^{1}, \quad\left[a_{1, i}, a_{2, i}\right]=\ell_{i}^{2},
$$

where we shall assume that

$$
\left|\ell_{j}^{i}\right| \leq 1, \quad i, j=1,2 .
$$

Just as in (3.3.4), by Lemma 2.3.1, we have

$$
S \times\left\{a_{i, j}\right\} \text { is an } L L(d)-\partial \square_{1}^{r} \subset Q, \quad i, j=1,2 .
$$

These can therefore be filled in by

$$
\begin{gathered}
B_{i, j}^{r} \text { is an } L L(d)-\square_{1}^{r} \subset Q, \\
\partial B_{i, j}^{r}=S \times\left\{a_{i, j}\right\}, \quad i, j=1,2,
\end{gathered}
$$

because of the property $\mathcal{F}_{r}$ of $Q$. We can then use the Lemma 2.3.2 and the property $\mathcal{F}_{r+1}$ to "fill in" the four "hollow" sides of the square. We have then $(i, j=1,2)$

$$
\begin{gathered}
B_{i, \cdot}^{r+1}, B_{\cdot, j}^{r+1} \text { are } L L(d)-\square_{1}^{r+1} \subset Q, \\
\partial B_{i, \cdot}^{r+1}=B_{i, 1}^{r} \cup B_{i, 2}^{r} \cup\left(S \times \ell_{i}^{1}\right), \\
\partial B_{\cdot, j}^{r+1}=B_{1, j}^{r} \cup B_{2, j}^{r} \cup\left(S \times \ell_{j}^{2}\right) .
\end{gathered}
$$

Let then

$$
\Delta=\bigcup_{i=1,2}\left(B_{\cdot, i}^{r+1} \cup B_{i, \cdot}^{r+1}\right) \cup(S \times L)
$$

If we use the Lemma 2.3.2 to control the last term of (3.3.29) and glue the pieces together we deduce that

$$
\Delta \text { is an } L L(d)-\partial \square_{1}^{r+2} \subset Q .
$$

Hence by the property $\mathcal{F}_{r+2}, \Delta$ can be filled in by

$$
\begin{gathered}
B^{r+2} \text { is some } L L(d)-\square_{1}^{r+2} \subset Q, \\
\partial B^{r+2}=\Delta .
\end{gathered}
$$


The above information (3.3.24)-(3.3.32) will be summarized, abusively, by the single notation

$$
\partial B^{r+2}=\operatorname{Rim}(S \times L) .
$$

iv) The 2-dimensional cylinder: The general case. The notations are as in 3.3.1.iii) but we no longer assume that $Q$ is split. We shall assume as in (3.3.15) that

$$
\left|\ell_{i}^{1}\right|,\left|\ell_{i}^{2}\right| \leq\left(\sup \left|a_{i j}\right|+10\right)^{-C},
$$

for some appropriate $C>0$. We make the construction of $B_{i, \cdot}^{r}$ and $B_{\cdot, i}^{r}$ as in (3.3.26), (3.3.27), (3.3.28), with the use of (3.3.34) as before and the same modifications for the proof as in 3.3.1.ii). We obtain thus $\Delta$ as in (3.3.29). To prove (3.3.30) we have to control the term $S \times L$. This is done by the Lemma 2.3.2 and the analog of the cascade of maps (3.3.17). Where now

$$
\Psi:[0,1] \times[0,1] \longrightarrow L
$$

is the two dimensional scaling map. The property $\mathcal{F}_{r+2}$ completes the construction of $B^{r+2}$ as in (3.3.31), (3.3.32), (3.3.33), as before.

v) The general cube: The split case. Let

$$
\begin{gathered}
F=\left[a_{1}, b_{1}\right] \times\left[a_{2}, b_{2}\right] \times \cdots \times\left[a_{t}, b_{t}\right] \subset V_{1}, \\
\left|b_{i}-a_{i}\right| \leq 1, \quad 1 \leq i \leq t,
\end{gathered}
$$

be some parallelepiped of $V_{1}$ with sides parallel to the first $t$-axes $(1 \leq$ $\left.t \leq \operatorname{dim} V_{1}\right)$ and diameter $\leq 1$. We shall then proceed exactly as in 3.3.1.i)-iii) and use property $\mathcal{F}_{r}$ to first fill in the $2^{t}$ "corner cubes": $S \times F_{0}, F_{0}$ being the vertices (i.e. 0 -faces of $F$ ). Then we use the previous construction and property $\mathcal{F}_{r+1}$ to fill in the $\operatorname{Rim}\left(S \times F_{1}\right)$ where $F_{1}$ are the 1-dimensional faces of $F$. By "fill in the Rim..." we mean that we perform the construction that is summarized by (3.3.12). And so on. We obtain at the end

$$
\begin{gathered}
B \text { some } L L(d)-\square_{1}^{r+t} \subset Q, \\
\partial B=\operatorname{Rim}(S \times F),
\end{gathered}
$$


where the same abusive notation for the term "Rim" is used to abbreviate the above construction. One thing that should be kept in mind is that (3.3.36) implies that

$$
S \times F \subset \partial B
$$

vi) The general construction. The way one eliminates the condition that $Q$ is a split group should now be quite clear. One simply uses the full thrust of Lemma 2.3.2. Scaling maps are considered as before

$$
\Psi:[0,1] \longrightarrow\left[a_{1}, b_{1}\right], \ldots, \Psi:[0,1]^{t} \longrightarrow F
$$

with $a_{1}, b_{1}, \ldots, F$ as in 3.3.1.v), and the condition

$$
\left|b_{i}-a_{i}\right| \leq\left(\sup _{i}\left(\left|a_{i}\right|,\left|b_{i}\right|\right)+10\right)^{-C}, \quad i=1, \ldots, t,
$$

is imposed on $F$. The details are as in 3.3.1.ii)-iv) and will be left to the reader.

The following comments on the constructions that we have made up to now are in order:

REMARKS.

i) The coordinates in $V_{1}$ and $V_{2}$ play different roles. The $V_{2}$ coordinates that admit non-trivial real roots, act on $N_{E}$ and form on $N_{E} \times V_{2}$ a "hyperbolic structure", i.e. we have $r-1$ "Hyperbolic sections". It is this that allows us to "shrink" metrically $\partial \square_{d}^{r}$ and embed it appropriately onto $S$, which is some $L L(d)-\partial \square_{1}^{r} \subset N_{E} \bowtie V_{2}$.

The $V_{1}$ coordinates that have trivial real roots "act as Euclidean rotations" on the space $N_{E} \bowtie V_{2}$. This fact is vital for the above construction and comes out through the Lemmas 2.3.1-2.

We use then the property $\mathcal{F}$ of $Q$ to fill in the "prisms" (or "Euclidean" cylinders if you prefer - or "food cans"!) obtained by $S$ and right translations by the extra coordinates coming from $V_{1}$.

ii) Questions of uniformity. In the above constructions $d$ was the free parameter (at the end we will let $d \longrightarrow \infty$ ). All the constants $C>0$ introduced in the above constructions did not therefore depend on $d$. It is important to note also that these constants $C>0$, in the construction 
in Section 3.3.1, did not depend on $a_{1}, b_{1}, a_{2}, b_{2}, \ldots, a_{i, j}, \ldots$ or $F$ of 3.3.1.i)-vi) either.

\subsubsection{Filling in a Large Cylinder.}

The construction of "filling in small cylinders" in 3.3.1, can be carried out for a "large cylinder" also

$$
S \times[-A, A]^{t} \subset\left(N_{E} \times V_{2}\right) \times V_{1} .
$$

The problem is the uniformity of Remark 3.3.1.ii). To avoid having to "drag in" the size, $A \gg 1$, of the large cylinder in the Lip-constants, we have to proceed differently.

i) The split case. Once more let us start with the case when $Q=$ $N \bowtie V$ is a split group, and let us subdivide $[-A, A]^{t}$ into unit cubes $F_{1}, F_{2}, \ldots, F_{p}\left(p \approx(2 A)^{t} ; F_{j}\right.$ is a $\left.\square_{1}^{t}, 1 \leq j \leq p\right)$. The idea is to "fill in" each $S \times F_{j}$ independently by

$$
\begin{gathered}
B_{j} \text { is an } L L(d)-\square_{1}^{r+t} \subset Q, \quad j=1, \ldots, p, \\
\partial B_{j}=\operatorname{Rim}\left(S \times F_{j}\right),
\end{gathered}
$$

and furthermore do so in such a way that these $B_{j}$ 's fit together like a "honeycomb" and that their union "fills" in the $\operatorname{Rim}\left(S \times[-A, A]^{t}\right)$.

Here the notions and the notations from the theory of currents can be used with profit (cf. Section 3.0, [12], [13]. We have to assume then that the appropriate orientations have been assigned to the corresponding spaces). With these notations we have

$$
\begin{gathered}
B=\sum_{j=1}^{p} B_{j}, \quad \partial B=\sum_{j=1}^{p} \partial B_{j}, \\
B \text { is an } L L(d)-\square_{1}^{r} \times[-A, A]^{t} \subset Q, \\
\partial B=\operatorname{Rim}\left(S \times[-A, A]^{t}\right),
\end{gathered}
$$

where, without attempting to give a formal definition of (3.3.41) we insist on the following consequences implied by (3.3.41) ( $c f$. Remark 
$3.1 .1)$

$$
\begin{gathered}
S \times[-A, A]^{t} \subset \partial B, \\
\partial B \cap \pi^{-1}\left[u \in V_{1},|u| \leq C\right] \\
=\left(S \times[-A, A]^{t}\right) \cap \pi^{-1}\left[u \in V_{1},|u| \leq C\right],
\end{gathered}
$$

where $\pi: Q=\left(N_{E} \bowtie V_{2}\right) \bowtie V_{1} \longrightarrow V_{1}$ is the canonical projection. For the validity of (3.3.43), as we shall see, we shall need to have $A \gg 1$ appropriately large.

To clarify matters we shall consider first the two cases $t=1, t=2$.

The case $t=1$. We subdivide

$$
[-A, A]=\bigcup_{j=-A}^{A-1} I_{j}, \quad I_{j}=[j, j+1], \quad-A \leq j<A
$$

and construct $B_{\text {left }}^{r}(j), B_{\text {right }}^{r}(j)$, i.e. $B_{a}^{r}, B_{b}^{r}$ with $a=j, b=j+1$ (as in (3.3.5)) for the "small cylinder" $S \times I_{j}$ as in (3.3.4)-(3.3.6). We also make the construction, as we may, so that

$$
B_{\text {right }}^{r}(j)=B_{\text {left }}^{r}(j+1), \quad-A \leq j \leq A-1 .
$$

We then construct

$$
\begin{gathered}
B_{j}^{r+1} \text { is an } L L(d)-\square_{1}^{r+1} \subset Q, \\
\partial B_{j}^{r+1}=B_{\text {left }}^{r}(j) \cup B_{\text {right }}^{r}(j) \cup\left(S \times I_{j}\right),
\end{gathered}
$$

$$
B^{r+1}=\cup B_{j}^{r+1}=\sum B_{j}^{r+1},
$$

where the $\sum$ refers to the notations from the theory of currents ( $c f$. Section 3.0, [12], [13]). It is then clear that

$$
B^{r+1} \text { is an } L L(d)-\left(\square^{r} \times[-A, A]\right),
$$

$$
\partial B^{r+1}=B_{\text {left }}^{r}(-A) \cup B_{\text {right }}^{r}(A-1) \cup(S \times[-A, A]),
$$

which is exactly what is meant by (3.3.41). The basic fact (3.3.43) is here clearly ensured by (3.3.44), (3.3.45), provided that

$$
A \geq C(\log d)^{C},
$$


for some appropriate $C>0$. Indeed this implies that the "left" and the "right" of the "can" (3.3.47) do not reach the central region because the canonical projection $\pi: Q \longrightarrow V_{1}$ is Lip (1). The uniformity in $A$ of the construction in (3.3.44) plays a crucial role here ( $c f$. Remark 3.2.1.ii)).

The case $t=2$. We subdivide

$$
[-A, A] \times[-A, A]=\bigcup_{i, j=-A}^{A-1}[i, i+1] \times[j, j+1],
$$

and start by filling in each of the four vertices $C_{\alpha}(i, j), 1 \leq \alpha \leq 4$, of $I_{i, j}=[i, i+1] \times[j, j+1]$

$$
\partial B_{\alpha}^{r}(i, j)=S \times C_{\alpha}(i, j), \quad \alpha=1,2,3,4 .
$$

This is done in a consistent way, i.e. if two neighbouring squares have a common vertex, we choose the same filling $\left(e . g . B_{1}^{r}(i, j)=B_{3}^{r}(i-1, j)\right.$ with obvious notation).

Having done that, we then fill in the hollow sides of each $S \times I_{i, j}$ (as in (3.3.26)-(3.3.29)) using the already constructed $B_{\alpha}^{r}(i, j)$. This is again done in a consistent way, i.e. two $S \times I_{i, j}$ that are side by side (or one on top of the other) must have their common side filled in an identical way. The final step is to construct $B^{r+2}(i, j)$ such that

$$
\begin{aligned}
& B^{r+2}(i, j) \text { is an } L L(d)-\square_{1}^{r+2} \subset Q \\
& \partial B^{r+2}(i, j)=\operatorname{Rim}\left(S \times I_{i, j}\right), \quad i, j=-A, \ldots, A-1 .
\end{aligned}
$$

Then, using again the notations from the theory of currents, we set

$$
B=\sum_{i, j} B^{r+2}(i, j)
$$

which is

$$
B \text { is an } L L(d)-\square_{1}^{r} \times[-A, A]^{2} \subset Q .
$$

The boundary $\partial B$ consists of exactly two parts

$$
(\partial B)_{1}=S \times[-A, A]^{2}, \quad(\partial B)_{2}=\partial B \backslash(\partial B)_{1},
$$

and clearly if $x \in(\partial B)_{2}$, then $x \in \partial B^{r+2}(i, j)$ where either $i$ or $j$ (or both) are equal to $-A$, or $A-1$. This, together with (3.3.48) and the 
uniformity in $A$ of the constants in (3.3.48), implies for the same reason as in the case $t=1$, that (3.3.43) holds if

$$
A \geq C(\log d)^{C},
$$

for some appropriate $C$.

The way one generalizes the above constructions to any $t \geq 1$, by filling in the unit subcubes $S \times[-A, A]^{t}$ in such a way that their successive $\partial_{r}, \partial_{r+1}, \ldots$ boundaries coincide, should be clear. We obtain thus the required $B$ that satisfies (3.3.40)-(3.3.43).

ii) The general case. When $Q$ is not necessarily split we have to modify the construction of the previous section at only one point:

Instead of subdividing $[-A, A]^{t}$ in (3.3.38) into unit cubes we subdivide it into $(2 A / \lambda)^{t}$ cubes $\left[a_{1}, b_{1}\right] \times\left[a_{2}, b_{2}\right] \times \cdots$ of size

$$
\lambda=\left|b_{j}-a_{j}\right| \leq(A+10)^{-C}, \quad j=1, \ldots, t .
$$

Each of these cubes is then filled in as in 3.3.1.vi), and this is done with consistent $\partial_{r+k}$-boundaries as before. Taking the union, or more accurately, summing the corresponding currents, we obtain as before

$$
\begin{gathered}
B=\sum_{i_{1}, \cdots=-A / \lambda}^{A / \lambda-1} B\left(i_{1}, \ldots, i_{t}\right), \\
B \text { is an } L L(d)-\square_{1}^{r} \times\left[\frac{-A}{\lambda}, \frac{A}{\lambda}\right]^{-t}, \\
\partial B=\operatorname{Rim}\left(S \times[-A, A]^{t}\right), \\
\partial B \cap \pi^{-1}\left(u \in V_{1},|u| \leq C\right) \\
=\left(S \times[-A, A]^{t}\right) \cap \pi^{-1}\left(u \in V_{1},|u| \leq C\right),
\end{gathered}
$$

where again $\pi: Q \longrightarrow Q / N \longrightarrow V_{1}$ is the canonical projection. The size $[-A / \lambda, A / \lambda]$ of the cube in $(3.3 .51)$ has been multiplied by $1 / \lambda$ because of the summation from $-A / \lambda$ to $A / \lambda-1$ in (3.3.50).

The key fact (3.3.53) is again guaranteed by a large enough choice of $A$

$$
A \geq C(\log d)^{C},
$$

and the fact that $\pi$, being a group homomorphism, is Lip (1). 
The final point of this construction is that the parameters are chosen so that

$$
A \sim C(\log d)^{C}, \quad \lambda \sim C(\log d)^{-C} .
$$

By the rescaling (3.0.6), (3.0.7), it follows therefore that $B$ in (3.3.51) is an $L L(d)-\square_{1}^{r+t}$.

\section{Proof of the Main Theorem $(C)$.}

In this section I shall give the proof of the $C$-part of the main Theorem. This is the difficult part of the theorem and it uses the algebraic and geometric constructions that we have developed in this paper. I shall give three different ways of making this last step. Basically all three stem from the same idea and it is only a matter of using a different language and different tools to put things together. That language and tools can be summarized as follows.

i) Transversality and Sard's theorem from Differential Topology. This is what we do in Section 4.5. 4.3 .

ii) Slicing from the theory of currents. This is what we do in Section

iii) We can globalize and avoid the explicit use of either of the above. We then only use the very simplest definitions from the theory of currents, but the price that we have to pay is that we have to keep track of the orientations and the signs of the currents involved. This is what is done in Section 4.2.

\subsection{Currents in Riemannian manifolds.}

Let $M$ be some Riemannian manifold, we shall recall some of the standard definitions and properties of currents on $M$. I shall deliberately, but abusively, ignore the questions of orientation. Some of the statements below are therefore as such, incomplete. The reader will have to fill in the details concerning the orientations on his own ( $c f$. $[12])$.

i) We denote by $\Lambda(M)$ the space of $C^{\infty}$ compactly supported forms on $M$ and by $\Lambda^{*}(M)$ the dual space of currents on $M$. We denote 
$\|\omega\|=\sup _{m}|\omega(m)|_{\Lambda}$ for the Riemannian norm $|\cdot|_{\Lambda}$ induced on $\Lambda T^{*} M$. Let $T \in \Lambda^{*}(M)$, we say that $T$ is an integration current of finite mass if $M(T)=\|T\|=\sup \{|\langle T, \omega\rangle|, \omega \in \Lambda,\|\omega\| \leq 1\}<+\infty,(M(T)$ is the notation used in [13]). current

ii) Every $\varphi: \square_{1}^{r} \longrightarrow M$ that is $\operatorname{Lip}(A)$ induces a unique integration

$$
T=\left[\varphi\left(\square_{1}^{r}\right)\right], \quad\langle T, \omega\rangle=\int_{\square_{1}^{r}} \varphi^{*}(\omega), \quad \omega \in \Lambda(M),
$$

of mass $\|T\| \leq(1+A)^{r}$. This is evident if $\varphi$ is $C^{\infty}$ and defined in some neighbourhood of $\square_{1}^{r}$. The extension to an arbitrary $\varphi$ as above is routine.

iii) Let $\Omega \subset M$ be some open subset. Then the injections $\Omega \longrightarrow M$, $\Lambda(\Omega) \subseteq \Lambda(M)$ defines canonically a restriction operator $\Lambda^{*}(M) \longrightarrow$ $\Lambda^{*}(\Omega)$. We shall use the notation $\left.T \rightarrow T\right|_{\Omega}$ for that operator and we have

$$
\left.(d T)\right|_{\Omega}=d\left(\left.T\right|_{\Omega}\right)
$$

Furthermore, if $T$ is an integration current, then $\left.T\right|_{\Omega}$ is also an integration current and

$$
\left\|\left.T\right|_{\Omega}\right\|=\left\|\chi_{\Omega} T\right\| \leq\|T\|,
$$

where $\chi_{\Omega}$ is the characteristic function of $\Omega$.

\subsection{The current interpretation of the Geometric construc- tions.}

For the proof of our main Theorem, we shall consider currents on the Riemannian manifold $Q=M$, where $Q$ is a soluble simply connected group assigned with its left invariant Riemannian structure. Let $\pi: Q \longrightarrow Q / N=V$ be the canonical projection, where $N$ is the nilradical, and let

$$
\Omega=\pi^{-1}\left(y \in V,|y|_{V}<1\right) \subset M .
$$

We shall then consider the restriction on $\Omega$ of the currents of $M$ as in 4.1.iii). We shall also consider the restriction of the Riemannian structure of $Q$ on $\Omega$ which gives a Riemannian structure that is quasiisometric with the product Riemannian structure

$$
N \times B^{s}=N \times\left\{y \in \mathbb{R}^{s},|y|<1\right\} .
$$


In (4.2.1) we assign the nilradical $N$ with its left invariant structure and the Euclidean unit ball $B^{s}$ with the Euclidean structure. Let now $B$ be the current defined in (3.3.39)-(3.3.43), (3.3.50)-(3.3.53) of Section 3.3.2, and where once more we choose to ignore all questions of orientation and of signs. As we shall see presently, the signs and the orientation are not essential for the proof of the main Theorem. If, however, we are prepared to go through the details and work out the correct signs at every point of the construction in Section 3.3.2, the proof in Section 4.3, becomes "cleaner" and simplifies.

We shall consider the integration current $[B]$ that is defined as in 4.1.ii) by $\Phi: \square_{1}^{r} \times[-A / \lambda, A / \lambda]^{\sigma} \longrightarrow Q$. Here $r$ is as in Section 3.2.4 if $N_{E}$ is abelian, and $r=2$ if $N_{E}$ is of Heizenberg type, in both cases $\sigma=\operatorname{dim} V_{1}$. One must recall that the construction of $B$ in 3.3.2 was done under the assumption that $Q$ satisfied $\mathcal{F}_{r}, \mathcal{F}_{r+1}, \ldots, \mathcal{F}_{r+\sigma}$ and the choice of $A, \lambda$ was such that $A / \lambda \sim C(\log d)^{C}(c f$. (3.3.54)). It follows therefore, that

$$
\|[B]\| \leq C(\log d)^{C},
$$

and therefore also that

$$
\left\|\left.[B]\right|_{\Omega}\right\| \leq C(\log d)^{C} .
$$

We shall consider $T=\partial\left(\left.[B]\right|_{\Omega}\right)$. By (3.3.43), (3.3.53), (3.2.27)-(3.2.30), 4.1.iii) it follows that if we suppose that $Q$ satisfies the $\mathcal{F}$ condition and is also a $C$-group (these two actions on $Q$ will presently be shown to be incompatible!) then

$$
\operatorname{supp} T \subset\left(S \times[-A, A]^{\sigma}\right) \cap \pi^{-1}[u \in V,|u|<1]=\bigcup_{\varepsilon= \pm 1} C\left(\varepsilon_{1}, \ldots, \varepsilon_{r}\right)
$$

where, with the identification of $\Omega$ with (4.2.1) and with the identification of $N$ with $\mathbb{R}^{m}$ (induced by the exponential coordinates of sections $2.1,2.2$ ) we set

$$
C\left(\varepsilon_{1}, \ldots, \varepsilon_{r}\right)=\left[x=\left(\varepsilon_{1} d, \ldots, \varepsilon_{r} d, 0, \ldots, 0\right), u \in V,|u| \leq C\right]
$$

where $\varepsilon_{1}, \ldots, \varepsilon_{r}= \pm 1$ ( $c f$. sections $3.2 .3,3.2 .4$ ). In the above notations we have assumed that $N_{E}$ is abelian. The changes that have to be made in the notations to deal with the case when $N_{E}$ is of Heizenberg type will be left to the reader. 
If in Section 3.3.2 we are prepared to go through the signs and the orientations involved in the "filling currents" of (3.3.39) and (3.3.50), we can show that

$$
T=\left.\partial[B]\right|_{\Omega}=\sum_{\varepsilon= \pm 1} \pm\left[C\left(\varepsilon_{1}, \ldots, \varepsilon_{r}\right)\right]
$$

where $[\cdot]$ indicates the integration on the $s$-dimensional chain that is Lip embedded in $\Omega$. We can give explicitely the \pm 's on (4.2.3) but this is irrelevant, these signes do not depend on $d$.

At this point we shall stop to point out that without "bothering" to go through the orientations and signs involved, what we have "for sure" is

$$
T=\left.\partial[B]\right|_{\Omega}=\sum f_{\varepsilon_{1}, \ldots, \varepsilon_{r}}\left[C\left(\varepsilon_{1}, \ldots, \varepsilon_{r}\right)\right]
$$

where for each fixed $\left(\varepsilon_{1}, \ldots, \varepsilon_{r}\right)$ we have

$$
\left|f_{\varepsilon_{1}, \ldots, \varepsilon_{r}}(y)\right|=1, \quad y \in B^{s} .
$$

Indeed the function $f_{\varepsilon_{1}, \ldots, \varepsilon_{r}}(\cdot)$ which "a priori" may depend on $d$ is what gives the correct sign on the subcubes of size $\lambda$ in (3.3.50). In particular, in the split case (3.3.39)-(3.3.43) when $\lambda=1$, we already have (4.2.3) without worrying about the orientations.

\subsection{The proof of the main theorem $C$ using and keeping track of the signs of the currents.}

From (4.2.2) and (4.2.3) (or (4.2.4)), we shall be able to draw a contradiction when $d \longrightarrow \infty$. This will prove the incompatibility of the $C$-condition and $\bigcap_{j=r}^{r+\sigma} \mathcal{F}_{j}$ and will complete the proof of the main Theorem $(C)$ because $r+\sigma=\operatorname{rank} Q+1$ and $r \geq 2$. To describe things in general terms, what will be proved is, that under the condition $(C)$ for $Q$ we cannot have a polynomial upper bound for $\phi_{n}(R)(n=$ $r, r+1, \ldots, r+\sigma)$ in the main Theorem $(C)$.

The fact that (4.2.2), (4.2.3) (as $d \longrightarrow \infty$ ) are contradictory is easy to see. Indeed let

$$
\omega=f\left(x_{1}, \ldots, x_{m}\right) g\left(y_{1}, \ldots, y_{s}\right) d y_{1} \wedge \cdots \wedge d y_{s}
$$


for some $f \in C_{0}^{\infty}(N), g \in C_{0}^{\infty}\left(B^{s}\right)$, then

$$
\begin{aligned}
&\langle\omega, T\rangle=C\left(\int g d y\right) \sum \pm f\left(\varepsilon_{1} d, \varepsilon_{2} d, \ldots, \varepsilon_{r} d, 0, \ldots, 0\right) \\
&|\langle\omega, T\rangle|=\left|\left\langle d \omega,\left.[B]\right|_{\Omega}\right\rangle\right| \\
& \leq\|d \omega\|\left\|\left.[B]\right|_{\Omega}\right\| \\
& \leq C(\log d)^{C} \sup _{\Omega}|d f|
\end{aligned}
$$

where $|d f|$ stands for the Riemannian norm in $\Omega$. Observe now that the mutual distances in $N$ of the points $\left(\varepsilon_{1} d, \ldots, \varepsilon_{r} d, 0, \ldots, 0\right)$ is greater or equal $C d^{\alpha}$ for some $\alpha>0$ (by the polynomial distortion between the distances in $N$ and the corresponding Euclidean distance induced by the exponential coordinates). It follows therefore that we can choose $f$ and $g$ so that the right hand side of (4.3.1) is greater or equal than 1 (no matter what the choice of the \pm 's is) and yet

$$
\sup _{\Omega}|d f| \leq C d^{-\alpha} .
$$

From this and (4.3.2), by letting $d \longrightarrow \infty$, we obtain the required contradiction. This completes the proof of our theorem.

The above proof can easily be modified so as to make (4.2.4) (and not (4.2.3)) the starting point. The only difference is that now

$$
\omega=f\left(x_{1}, \ldots, x_{m}, y_{1}, \ldots, y_{s}\right) d y_{1} \wedge \cdots \wedge d y_{s},
$$

where the dependence of the coefficient $f \in C_{0}(\Omega)$ on $y \in V$ is designed to compensate for the sign of $f_{\varepsilon_{1}, \ldots, \varepsilon_{r}}$ in (4.2.4). We can again choose $f$ so that in (4.3.1) we have

$$
|\langle T, \omega\rangle| \geq 1
$$

and yet

$$
\sup _{\Omega}|d \omega| \leq C d^{-\alpha}
$$

Observe that now (4.3.4) is not obtained though the control (4.3.3) of $d f$. What allows us to assert (4.3.4) is the fact that $d \omega$ only involves the partial derivatives $\partial / \partial x_{j}$ of $f$. 


\subsection{The proof of the main Theorem $(C)$ using the slicing.}

This is but a variant of the previous proof of the main Theorem of Section 4.3. It relies on the non trivial notion of the slicing of currents (cf. [13, Section 4.3]). This slicing operation allows us to define for almost every $x \in B^{s}$ in (4.2.1), a current $\langle[B], \pi, x\rangle$ on $N$, which is the "slice" of $[B]$ with $\pi^{-1}(x) \subset Q$. Here we shall identify $\pi^{-1}(x)$ with $N$ and use the notations of $[13$, Section 4.3$]$ and the fact that $[B] \in \Lambda_{\delta}^{*}$, (i.e. as a current it acts on $\delta$-forms) where $\delta=r+\sigma=\operatorname{dim} B$ (with the notations of Section 3.3.2, cf. (3.3.51)). The construction and the formalism of this slicing depends on the fact that the dimension of the current (equal to $r+\sigma$ ) is greater or equal than the dimension of the target space of $\pi$, cf. Section 2.1. This formalime is non-trivial and I shall refer the reader to [13, Section 4.3] for the details.

At any rate, if we are prepared to use this notion of slicing, we can obtain a contradiction between the conditions $(\mathcal{F})$ and $(C)$ on the group $Q$, starting this time from the weaker (4.2.4). The advantage of this approach lies therefore in the fact that we do not have to "chase around" orientations and signs of currents.

The contradiction is obtained by a very similar argument as the one used in Section 4.3, but which is now localized to each individual $N$-fiber of (4.2.1). Indeed the polynomial distance distortion in $N$, together with the automatic control of $\partial\langle[B], \pi, x\rangle$ that we have from (4.2.4), will give the following lower bound of the total mass

$$
M[\langle[B], \pi, x\rangle] \geq C d^{\alpha}, \quad \text { almost all } x \in B^{s} .
$$

The contradiction now is obtained between (4.4.1), (4.2.2) and [13] Theorem 4.3.2.

The idea of the above variant of the proof is, of course, very simple: Instead of restricting $[B]$ to the cylinder $\pi^{-1}(|u|<1)$ we restrict it to the fiber $\pi^{-1}(u)$ (fixedu $\left.\in V,|u| \leq 1\right)$. This allows us to ignore the possible variations of sign of $f_{\varepsilon_{1}, \ldots, \varepsilon_{r}}(y)= \pm 1$ in (4.2.5). The price we have to pay is that now we have to integrate with respect to $u$ in $(|u| \leq 1)$. [13, Theorem 4.3.2] allows us to perform the integration and obtain the contradiction. 


\subsection{The smooth fillings and an alternative proof of the main Theorem $(C)$. An outline.}

We have already pointed out at the end of Section 3.2.3 how in the first basic construction of $S \subset \mathbb{R}^{r} \bowtie \mathbb{R}^{r-1}$, which is an $L L(d)-\partial \square_{1}^{r} \subset$ $\mathbb{R}^{r} \bowtie V$, we can make $S$ to be a $C^{\infty}$ embedded $(r-1)$-dimensional sphere. Once this construction was made smoothly, we can proceed and do all the "fillings" of Section 3.3 in a smooth fashion.

To fix ideas, if we assume that the group $Q$ satisfies the condition $\mathcal{F}_{r}$, we can modify the filling of $S$ and construct

$$
\begin{array}{cl}
F: B^{r} \longrightarrow Q, & F \in L L(d) \cap C^{\infty}, \\
F\left(\partial B^{r}\right)=S, & F\left(B^{r}\right)=B \subset Q,
\end{array}
$$

where $B^{r}$ is the unit $r$-ball and where $F$ induces an embedding of $B^{r}$. This can be done by the usual Whitney perturbation technique (that can easily be adapted to leave the boundary fixed, cf. [14]) provided that

$$
\operatorname{dim} Q \geq 2 r+1
$$

The condition (4.5.1) is not, of course, a priori verified and the first thing that has to be done is to replace $Q$ by the direct product group $Q \times \mathbb{R}^{A}=Q_{A}$ (for some appropriate $A \geq 0$ ). By spilling out of $Q$ into $Q_{A}$ as little as we like, we can then make sure that $F$ is an embedding. Observe that the extra factor of $Q_{A}$ goes in the nilradical.

Let us also assume for simplicity that in Section 3.3 (with the notations that we used in Section 2.2) $V_{1}=0$, so that in the constructions of Section 3.3, after we fill in $S$, we stop. And we do not have to worry about the $\operatorname{Rim}\left(S \times[-A, A]^{\sigma}\right)$ (i.e. $\left.\sigma=0\right)$. We can then finish the proof of our main theorem with an obvious use of Transversality in a few lines.

Indeed, if we denote by $\pi: Q_{A} \longrightarrow V$ the canonical projection, where with our previous notations $V=V_{2}=Q / N$, by Sard's theorem, for almost all $y \in V, \pi^{-1}(y) \cap B$ is a 1-dimensional $\partial$-manifold and $\partial\left(\pi^{-1}(y) \cap B\right)=S \cap \pi^{-1}(y)$ (cf. [16]). The set $S \cap \pi^{-1}(y)$, when $|y| \leq 1$, is completely determined by the construction of $S$ and the mutual distance of its $2^{r}(r=2, \ldots)$ points in the Riemannian manifold $\pi^{-1}(y)$ (i.e. for the induced Riemannian structure in $\pi^{-1}(y) \subset Q_{A}$ ) is at least $C d^{\alpha}$. This holds for the same reasons as in sections 4.3 and 4.4, 
cf. (4.4.1). The conclusion for the 1-dimensional Hausdorff measure induced by the Riemannian structure of $Q_{A}$ is

$$
\operatorname{Vol}_{1}\left[\pi^{-1}(y) \cap B\right] \geq C d^{\alpha} \text {. }
$$

From this a lower estimate

$$
\operatorname{Vol}_{r}(B) \geq C d^{\alpha}
$$

follows at once ( $c f$. [13, Section 3.2]. In fact, using elementary differential calculus, we can easily see how (4.5.2) implies (4.5.3) directly). This clearly contradicts (4.2.2) and, once more, gives a proof to our Main Theorem.

Observe also, that in the above case, $\operatorname{dim} Q \geq 2 r-1$, so it is probably possible to use the "difficult" Whitney immersion theorem [14] and avoid the use of $Q_{A}$.

The above proof is very analogous to the proof given in Section 4.4. We can indeed say that here the notion of the slicing is picked up by Sard's theorem and Transversality, and what replaces [13, 4.3.2] is the fact that (4.5.2) implies (4.5.3).

This approach, through elementary differential topology, can be generalized in the general case, i.e. when $V_{1}$ is not necessarily zero. One then has to carry out the filling constructions of Section 3.3 and make sure that the integration currents that we use for the fillings are $\partial$-manifolds. This again is achieved by the Whitney approximation technique, applied to the manifold $Q_{A}(A \gg 1)$, but is more involved. I will not give the details. The reader who wishes to carry these details out for himself should observe the following point:

Already in 3.3.i) the current in (3.3.8) is not the boundary of a smooth manifold, even though $B_{a}, B_{b}$ have been chosen to be generic $C^{\infty} \partial$ - manifolds. The current in (3.3.8) is the boundary of a manifold with corners in the sense of [15]. This means that either we have to make the constructions in Section 3.3 using manifolds with corners, or do something at every step of the construction of Section 3.3 to "smooth" out these corners. Both these aproaches work. But I do not have the stomach to write the details down here.

REMARK. There is a varient of the Main Theorem that can be formulated as follows:

Let us say that a Riemannian manifold as in Section 0.2 has the property $\left(\mathcal{G}_{p}\right)(c f$. Section 3.0$)$, if there exists $C>0$, such that for all 
$R>10$ and all $\Phi: \partial \square_{1}^{p} \longrightarrow M(\Phi \in \operatorname{Lip})$ and such that $\operatorname{Vol}_{p-1}\left(\Phi\left(\partial \square_{1}^{p}\right)\right)$ $\leq R$ there exists $\hat{\Phi}: \square_{1}^{p} \longrightarrow M(\hat{\Phi} \in \operatorname{Lip})$ such that

$$
\left.\hat{\Phi}\right|_{\partial \square_{1}^{p}}=\Phi, \quad \operatorname{Vol}_{p} \hat{\Phi}\left(\square_{1}^{p}\right) \leq R^{C}, \quad R>10 .
$$

For a mapping $\Phi$ that is not $1-1$, the above definition of $\operatorname{Vol}_{r}$ has to be done with multiplicity (e.g. [29, p. 58]). The proof that we gave in this paper can be adapted to prove that the $C$-condition on the soluble simply connected Lie group $Q$ and the conditions $\mathcal{G}_{p}(2 \leq p \leq$ rank $Q+1)$ are not compatible.

In the above adaptations no new ideas are involved but the details are tedious and long. These details remain to be written out.

The difficulty in adapting the above proof lies in the Second Basic Construction of Section 3.3 and especially in the argument that was needed to supply a proof of (3.3.43). This variant of the Main Theorem is related to the Homological classification of Section 0.3.

\section{The Proof of the NC-Theorem.}

\subsection{Homotopy retracts on Lie groups.}

Let $Q$ be some simply connected soluble Lie group assigned with its left invariant Riemannian structure. In this section we shall construct $H(g, t) \in Q(0 \leq t \leq 1, g \in Q)$ appropriately smooth $\left(C^{\infty}\right.$ or at least Lip) homotopy retracts

$$
H(g, 0)=e, \quad H(g, 1)=g, \quad g \in Q,
$$

that have one of the following additional properties (or both)

$$
\begin{aligned}
& |d H| \leq C \exp (C|g|), \quad g \in Q, \\
& |d H| \leq C(|g|+C)^{C}, \quad g \in Q,
\end{aligned}
$$

where $|g|=d(e, g)$ is the Riemannian distance in $Q$ and where $[0,1] \times Q$ is assigned with the product Riemannian structure. We shall prove:

Theorem. Let $Q$ be as above, then a homotopy retract that satisfies (Exp.) always exists. A homotopy retract that satisfies (Pol.) exists on $Q$ if and only if $Q$ is an NC-group. 
Only the "if" part will be proved in this section. The "only if" part is a consequence of Section 4.

\subsection{Exponential coordinates.}

Let $N$ be some simply connected nilpotent group. We can define then the bijective mapping $\operatorname{Exp}=\operatorname{Exp}_{1}: \mathfrak{n} \longrightarrow N$ that gives on $N$ the exponential coordinates of the first kind ( $c f .[1])$,

$\operatorname{Exp}\left(x_{1} X_{1}+\cdots+x_{n} X_{n}\right) \in N, \quad\left(x_{1}, \ldots, x_{n}\right) \in \mathbb{R}^{n}, \quad n=\operatorname{dim} N$,

where $\mathfrak{n}$ is the Lie algebra of $N$. When $Q$ is a simply connected soluble group this mapping is not in general globally bijective and therefore it is not well suited to give coordinates on the group. We can use then $\operatorname{Exp}_{2}$ the exponential coordinates of the second kind ( $c f$. [1, Section 3.18]).

Using the above bijections, we can transport the radial homotopy retract of $\mathbb{R}^{n}\left(F(x, t)=t \cdot x, 0 \leq t \leq 1, x \in \mathbb{R}^{n}\right)$ to a homotopy retract

$$
R_{i}=\operatorname{Exp}_{i} \circ F \circ \operatorname{Exp}_{i}^{-1}, \quad i=1,2,
$$

(this notation is slightly abusive but clear enough) on $N$ or $Q$ as above. It is also very easy and standard to prove that when $N$ is nilpotent both $R_{1}, R_{2}$ satisfy (Pol.) More generally, when all the roots $\lambda_{1}, \lambda_{2}, \ldots$ of $Q$ are pure imaginary (i.e. there are no non-zero real roots and $\left(L_{1}, L_{2}, \ldots\right)=\varnothing$ in [2, Section 1.2]) then $R_{2}$ satisfies (Pol.). This fact is less standard but is very easy to verify because only the $\sin \theta$ and the $\cos \theta$ of the corresponding coordinates $\theta \in Q / N$, crop up in the multiplication $c f$. [10].

The construction of a homotopy retract that satisfies (Exp.) for a general simply connected soluble Lie group $Q$ is also very easy. Indeed, if the exponential coordinates are chosen as in sections 2.1, 2.2 so that $e_{1}, \ldots, e_{m} \in \mathfrak{n}, u_{1}, \ldots, u_{s} \in \mathfrak{h}$, the fact that both $\mathfrak{n}$ and $\mathfrak{h}$ are nilpotent, allow us to estimate polynomially both

$$
d\left(\operatorname{Exp}\left(\tau_{1} u_{1}\right) \cdots \operatorname{Exp}\left(\tau_{s} u_{s}\right)\right), \quad d\left(\operatorname{Exp}\left(t_{1} e_{1}\right) \cdots \operatorname{Exp}\left(t_{m} e_{m}\right)\right) .
$$

We also have (cf. [11])

$$
\begin{gathered}
t=\left|t_{1}\right|+\cdots+\left|t_{m}\right| \leq C(\exp C|g|+C), \\
\tau=\left|\tau_{1}\right|+\cdots+\left|\tau_{s}\right| \leq C(|g|+C), \\
g \in Q, \quad g=\left(t_{1}, \ldots, t_{m}, \tau_{1}, \ldots, \tau_{s}\right) .
\end{gathered}
$$


This, if we take into account that the action of $\operatorname{Ad}(g)$ on $T N$ has a norm that is at most $C(t+1)^{C} \exp (C \tau)$, easily completes the proof of (Exp.). The details will be left as an exercise to the reader.

One should observe, that if $M$ is some $C^{\infty}$ manifold, and if $\phi_{i}$ : $M \longrightarrow Q(i=1,2)$ are two mappings, then their group product satisfies

$$
d\left(\phi_{1} \cdot \phi_{2}\right)=d L_{\phi_{1}} \circ d \phi_{2}+d R_{\phi_{2}} \circ d \phi_{1}
$$

where $L$ and $R$ denote left and right translations on the group. If we identify $T_{g} Q$ with $T_{e} Q=\mathfrak{q}$ by left translation, this says that ( $c f .[26]$ )

$$
d\left(\phi_{1} \cdot \phi_{2}\right)=d \phi_{2}+d R_{\phi_{2}} \circ d L_{\phi_{2}}^{-1} \circ d \phi_{1}=d \phi_{2}+\operatorname{Ad} \phi_{2} \circ d \phi_{1} .
$$

\subsection{The semidirect product.}

Let now $Q=Q_{1} \bowtie Q_{2}$ be a semidirect product, where both $Q_{1}, Q_{2}$ are simply connected soluble groups, and let $H_{i}$ be a homotopy retract of $Q_{i}(i=1,2)$. Let

$$
g=q_{1} \cdot q_{2}, \quad q_{i} \in Q_{i}, \quad i=1,2,
$$

so that

$$
\begin{gathered}
q_{2}=\varphi_{2}(g), \quad q_{1}=g \cdot\left(\varphi_{2}(g)\right)^{-1}=\varphi_{1}(g), \\
\left|d \varphi_{2}\right| \leq 1, \quad\left|d \varphi_{1}(g)\right| \leq C \exp (C|g|) \\
\left|q_{1}\right|_{Q_{1}} \leq C \exp (C|g|) .
\end{gathered}
$$

On the other hand, if we denote by

$$
H(g, t)=H_{1}\left(q_{1}, t\right) \cdot H_{2}\left(q_{2}, t\right), \quad 0 \leq t \leq 1,
$$

and by

$$
\mathrm{Id}=\pi_{1} \oplus \pi_{2}: T Q \longrightarrow T Q_{1} \oplus T Q_{2},
$$

the orthogonal decomposition of $T Q$ induced by a basis of $\mathfrak{q}=\mathfrak{q}_{1} \bowtie \mathfrak{q}_{2}$ that consists of a basis of $\mathfrak{q}_{1}$ and a basis of $\mathfrak{q}_{2}$ (cf. sections 2.3 and 4 ), we have

$$
\begin{gathered}
\pi_{1} \circ d H=\operatorname{Ad}_{\mathfrak{q}_{1}}\left(H_{2}\right) \circ d H_{1} \circ d \varphi_{1}, \\
\pi_{2} \circ d H=d H_{2} \circ d \varphi_{2},
\end{gathered}
$$


with the obvious indentifications and obvious notations. It follows, in particular, that if $\mathrm{H}_{2}$ satisfies the condition (Pol.) of Section 5.1 then

$$
\left|\pi_{2} \circ d H\right| \leq C|g|^{C}+C
$$

Similarly, if $H_{1}$ satisfies (Pol.) of Section 5.1, and if $H_{2}$ has been constructed to satisfy the additional condition

$$
\left|H_{2}(g, t)\right|_{Q_{2}} \leq C|g|_{Q_{2}}+C, \quad g \in Q_{2}, \quad 0 \leq t \leq 1
$$

then (5.3.1) implies that

$$
\left|\pi_{1} \circ d H\right| \leq C \exp (C|g|)
$$

If $Q_{2}=N$ is nilpotent it is easy to see (5.3.4). This is because we can use exponential coordinates of the second kind $\left(x_{1}, \ldots, x_{n}\right)$ such that the ball of radius $r$ in $N$ is equivalent (in the obvious sense) to $\left\{\left|x_{j}\right| \leq r^{a_{j}}, 1 \leq j \leq n\right\}$ where $a_{j} \geq 1(1 \leq j \leq n)$ are integers. It follows that the retract $R_{2}$ of (5.2.1) satisfies (5.3.4). The same type of argument works if $Q_{2}$ has polynomial volume growth (cf. [10]), but this is less easy to see. (Alternatively, there exists $T \cong \mathbb{T}^{m}$ and a semidirect product $G=Q_{2} \bowtie T$ such that there exists $N \subset G$ that is a closed nilpotent, simply connected normal subgroup and such that $G=N T$ $N \cap T=\{e\}, c f .[27]$. This allows us to transfer the problem from $Q_{2}$ to $N)$.

The above facts are not essential and the details will be left as an exercise to the reader.

\subsection{NC-groups.}

Let $Q$ be some simply connected $N C$-group and let

$$
Q=N_{R} \bowtie Q_{R}=Q_{1} \bowtie Q_{2},
$$

be the semidirect decomposition induced by the corresponding algebra decomposition $\mathfrak{q}=\mathfrak{n}_{R} \bowtie \mathfrak{q}_{R}$ (cf. [2, Section 1.3]). We shall first construct the corresponding $H=H_{1} \cdot H_{2}$ that satisfies (5.3.1), (5.3.2).

Let us now fix $Y \in \mathfrak{q}_{R}$ such that

$$
L_{j}(Y) \leq-C_{0}, \quad j=1,2, \ldots, k
$$


for the real roots $L_{1}, \ldots, L_{k}$ (I use here the notation of [2, Section 1.3]), and let us define

$$
\begin{gathered}
\sigma(t)=\sigma(g, t)=\operatorname{Exp}\left(t\left(|g|^{C_{1}}+1\right) Y\right) \in Q \\
H_{p}(g, t)=H(g, \varphi(t)) \cdot \sigma(\psi(t)), \quad 0 \leq t \leq 1, \quad g \in Q
\end{gathered}
$$

where "." indicates group multiplication in $Q$ and where $\varphi, \psi \in$ $C^{\infty}([0,1])$ satisfy the following conditions:

$$
\begin{gathered}
\psi(t)= \begin{cases}0, & t=0,1, \\
1, & t \in[c, 1-c], \\
C^{\infty}, & \text { and monotone in between },\end{cases} \\
\varphi(t)= \begin{cases}0, & t \in[0,2 c], \\
1, & t \in[1-2 c, 1], \\
C^{\infty}, & \text { and increasing "almost linearly" in between } .\end{cases}
\end{gathered}
$$

From the above definition it is evident that

$$
\left|d H_{p}\right| \leq C\left(|g|^{C}+C\right), \quad t \in[0,2 c] \cup[1-2 c, 1] .
$$

It also follows from Section 5.3 that

$$
\left|d H_{p}\right| \leq\left|\operatorname{Ad}_{\mathfrak{q}_{R}}(\sigma)\right|\left(C|g|^{C}+C\right)+\left|\operatorname{Ad}_{\mathfrak{n}_{R}}\left(H_{2} \cdot \sigma\right)\right| \exp (C|g|+C) .
$$

It follows therefore that if $C_{0}>0$ in (5.4.1) and $C_{1}$ in (5.4.2) (in fact if you believe (5.3.4), you can take $\left.C_{1}=1\right)$ are chosen appropriately then the above retract $H_{p}$ satisfies (Pol.) because

$$
\left|\operatorname{Ad}_{\mathfrak{n}_{R}}(\sigma)\right| \leq C \exp \left(-C|g|^{C_{1}}\right), \quad 2 c \leq t \leq 1-2 c
$$

(cf. [2, Section 1.5] and [26]). This completes the proof of our Theorem.

\section{REMARKS.}

i) The reader who wants to make things even easier could consider a variant of the above construction which consists in setting

$$
\sigma(t)=\operatorname{Exp}\left(t R_{0}^{C} Y\right)
$$


with $R_{0} \gg 1$ and $Y \in \mathfrak{q}_{R}$ as before. One can then define

$$
H_{p}(g, t)=H(g, \varphi(t)) \cdot \sigma(\psi(t))
$$

( $\left.0 \leq t \leq 1, g \in Q,|g| \leq R_{0}\right)$ which is a retract of $B\left(R_{0}\right)$, the ball of radius $R_{0}$ in $Q$. This is weaker than our original construction but is good enough for most of our purposes.

ii) In the opposite direction, the proof of the $C$-part of the main theorem, if analyzed carefully, shows that the "exponential distortion" is optimal for the retracts of the $R$-balls $B(R)$. More precisely, if $G$ is a $C$-group, then there exists $c>0$ such that if

$$
H_{R}: B(R) \times[0,1] \longrightarrow B(R), \quad R \geq 1,
$$

are retracts, then

$$
\sup _{1<R<R_{0}}\left\|H_{R}\right\|_{\text {Lip }} \geq \exp \left(c R_{0}\right), \quad R_{0} \geq 1 .
$$

iii) If we use the fact that $N_{R}$ is of "strict exponential distortion" in $Q$ (cf. [26]), we can easily construct the above homotopy $H_{p}$ so that it satisfies the additional condition

$$
\left|H_{p}(g, t)\right|_{Q} \leq C|g|_{Q}+C, \quad g \in Q, 0<t<1 .
$$

\section{References.}

[1] Varadarajan, V. S., Lie groups, Lie algebras and their representations. Prentice Hall, 1984.

[2] Varopoulos, N. Th., Analysis on Lie groups. Revista Mat. Iberoamericana 12 (1996), 791-917.

[3] Varopoulos, N. Th., Mustapha, S., Forth coming book. Cambridge University Press.

[4] Hochschild, G., The structure of Lie groups. Holden-Day, 1965.

[5] Gromov, M., Geometric group theory. G. Niblo, M. Roller. LMS Lecture notes series 181. Cambridge University Press, 1991.

[6] Gromov, M., Filling Riemannian manifolds. J. Differential Geom. 18 (1983), 1-147.

[7] Raghunathan, M. S., Discrete subgroups of Lie groups. Springer Ergebnisse 68, 1972. 
[8] Gersten, S., Combinatorial and Geometric Group Theory (Editors: Duncan, Gilbert \& Howie) L.M.S. Lecture Notes Series 204 C.U.P., 1991.

[9] Jacobson, N., Lie algebras. Interscience, 1962.

[10] Alexopoulos, G., An application of homogenization theory to harmonic analysis: Harnack inequalities and Riesz transforms on Lie groups of polynomial growth. Canad. J. Math. 44 (1992), 691-727.

[11] Varopoulos, N. Th., Distance distortion on Lie groups. Institute MittagLeffler Report 31, 1995/96, and Symposia Math. XXXIX (1999), 320348.

[12] de Rham, G., Variétés différentiables. Hermann, 1960.

[13] Federer, H., Geometric Measure Theory. Springer Verlag, 1969.

[14] Whitney, H., The Self-intersection of a smooth $n$-manifold in $2 n$-space. Ann. of Math. 45 (1944), 220-293.

[15] Douady, A., Hérault, L., Arrondissement des variétés à coin. (Appendice to a Borel-Sene paper). Comm. Math. Helv. 48 (1973), 484-491.

[16] Hirsch, M. W., Differential Topology. Springer-Verlag, 1976.

[17] Varopoulos, N. Th., Saloff-Coste, L., Coulhon, T., Analysis and Geometry on Groups. Cambridge University Press, 1992.

[18] Varopoulos, N. Th., Diffusion on Lie groups I, II, III. Canad. J. of Math. 46 (1994), 438-448, 1073-1093, 48, 641-672.

[19] Stein, E. M., Singular Integrals and Differentiability Properties of Functions. Princeton Univ. Press, 1970.

[20] Bougerol, Ph., Example de théorèmes locaux sur les groupes resolubles. Ann. Inst. H. Poincaré XIX (1983), 369-391.

[21] Varopoulos, N. Th., The local thorem for symmetric diffusion on Lie groups. An overview. C.M.S. Conference Proceedings 21 (1997), 143152.

[22] Guivarc'h, Y., Croissance polynomiale et périodes des fonctions harmoniques. Bull. Soc. Math. France 101 (1973), 333-379.

[23] Reiter, H., Classical harmonic analysis and locally compact groups. Oxford Math. Monograph, 1968.

[24] Humphreys, J., Linear Algebraic Groups. Springer-Verlag, 1987.

[25] Montgomery, D., Zippin, L., Topological Transformation Groups. Interscience Tracts in Pure and Applied Math. No. 1, 1955.

[26] Varopoulos, N. Th., Geometric and Potential theoretic results on Lie groups. To appear in Canad. J. of Math.

[27] Auslander, L., Green, L. W., G-induced flows. Amer. J. Math. 88 (1966), 43-60.

[28] Varopoulos, N. Th., Analysis in Lie groups (II). To appear. 
136 N. Th. VARopoulos

[29] Lawson, H. B. Jr., Lectures on minimal submanifolds. Publish or Perish, Inc., 1980.

[30] Klingenberg, W., Lectures on Closed Geodesics. Springer-Verlag, 1978.

Recibido: 10 de agosto de 1.998

Nick Th. Varopoulos I.U.F. and

Departement de Mathématiques Université de Paris VI 75005 Paris, FRANCE 